
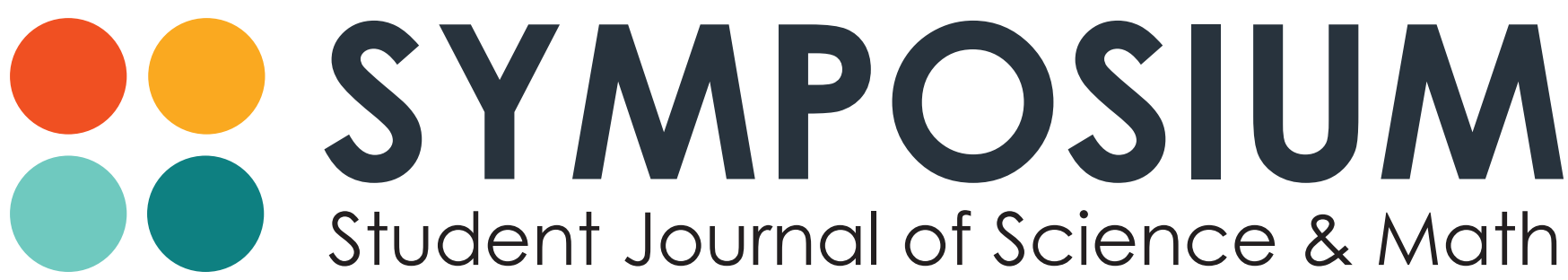
Student Journal of Science \& Math volume 2 Issue 1 


\section{MAPPING THE MOVEMENTS OF OVERWINTERING WESTERN MONARCH BUTTERFLIES (DANAUS PLEXIPPUS) AT THE PISMO BEACH MONARCH BUTTERFLY GROVE USING ARCGIS SOFTWARE}

A RESEARCH ARTICLE

by Brett Johnson, Jesse Wycko, Daniel Goldthwaite, and Tyler Brown

\section{Purpose}

Dr. Villablanca of the Cal Poly Biology Department commissioned this project with the goal of tracking Monarch Butterfly spatial redistribution in anticipation of or response to severe weather events. We believe that Monarchs cluster non-preferentially on introduced Eucalyptus trees until midwinter when they begin to cluster preferentially on native conifers. Based on the efforts of a previous group of students, it has been determined that, over a two-week time period in mid-winter, Monarchs spend the majority of their time on native conifers. We set out to clearly demonstrate Monarch Butterfly spatial redistribution in either anticipation of or response to severe weather.

\section{Overview}

Severe weather events occurred on October 3I,20I4 when I.04" of rainfall fell on Pismo Beach in a 24hour period. A cold front and light showers moved over San Luis Obispo County on Nov. I3, and on Nov. I9, about I/IO" fell on the Pismo Coastal area. Relative to the span of our study, low barometric pressure was observed around Nov. I and Nov. Io to II (Fig. I). 

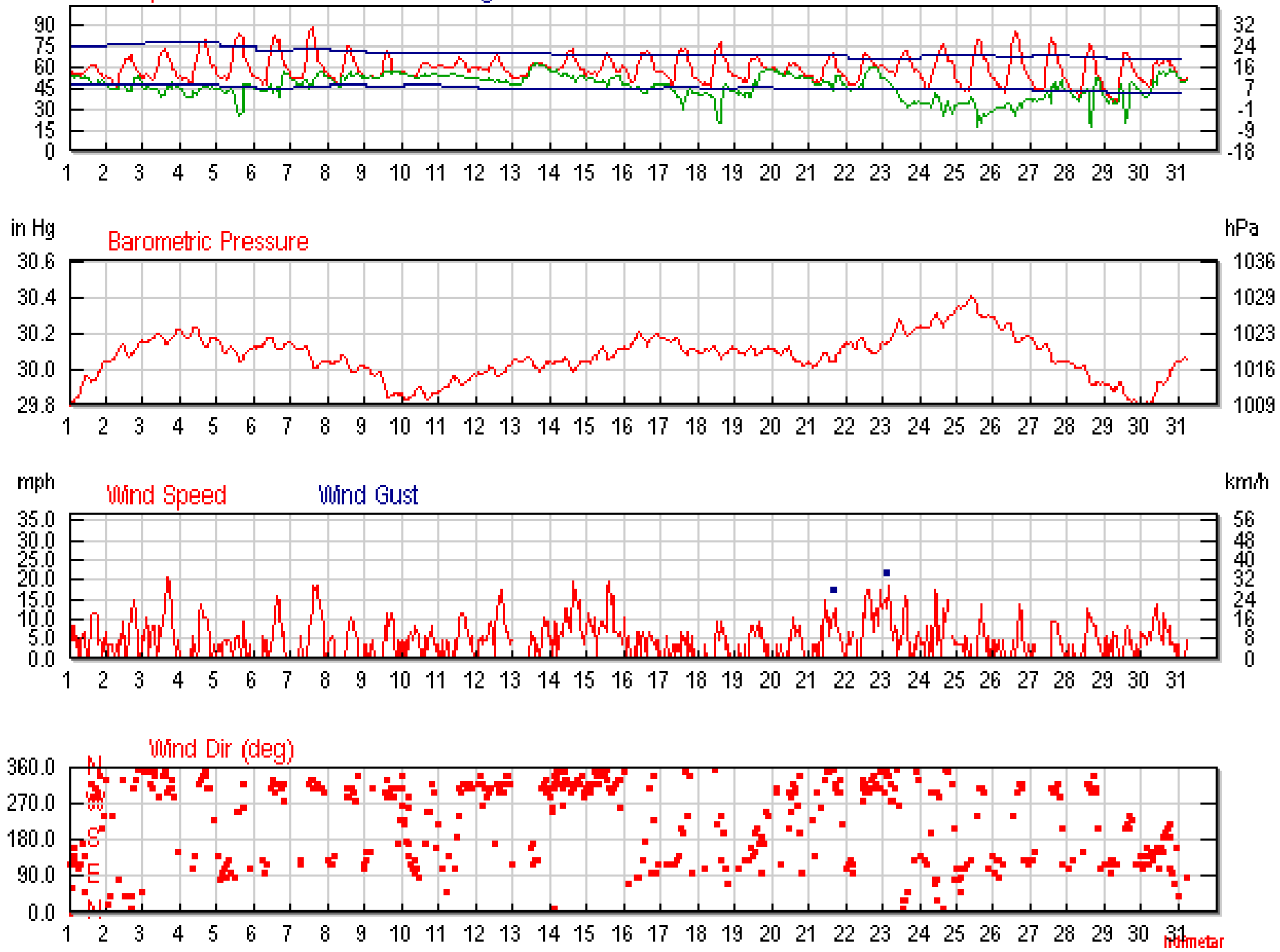

Figure I. Meteorological data for the Pismo area November 2014 (weatherunderground.com). 


\section{Methods}

\section{Equipment:}

- Binoculars

- Rangefinder

- Data sheets

- Anemometer

- Spotting telescope

- Pismo Grove map
o Major Grid (Fig. 3)
o Minor Grid (Fig. 4)

Data was collected by teams of I to 4 researchers at sunrise for 23 consecutive days, beginning Nov. I, 2014 and ending Nov. 23, 20I4. Using standardized counting techniques taught to us by State Parks Interpreter Danielle Patterson, we estimated the size, location, and height of each cluster of butterflies observed in the Pismo Monarch Butterfly Grove (PMBG) (Fig. 2).

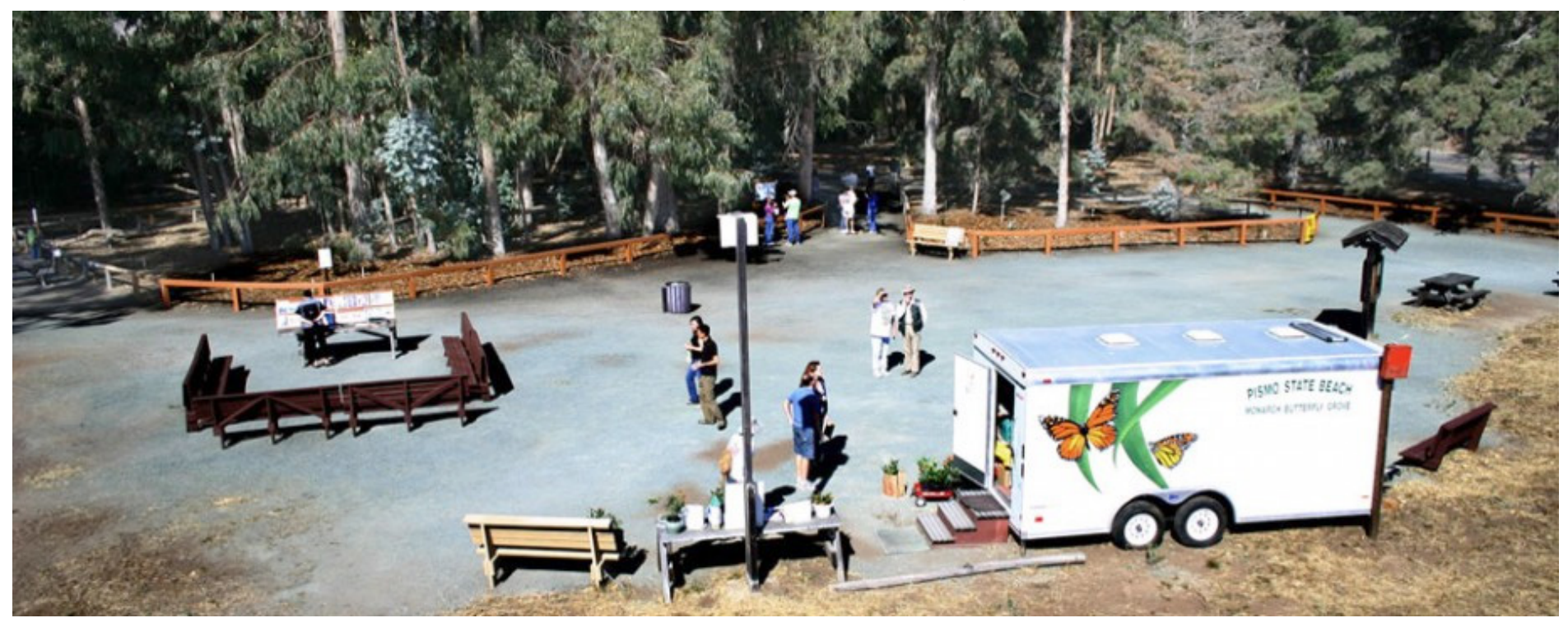

Figure 2. Pismo Monarch Butterfly Grove

Each cluster of butterflies was assigned a height estimate at the top and bottom of the cluster to the nearest meter. Hand-held rangefinders were used to determine this height value from directly underneath the cluster. A count estimate was then performed by two individuals so an average value could be assigned. We limited our estimations to a $20 \%$ margin of error between counter or a recount was performed. Using previously generated grid maps (created by a previous group of students), we determined the individual trees on which each cluster of butterflies was located. We first examined the Major Grid (Fig. 3) and decided which Major Cell the cluster resided in. Each Major Cell contained a Minor Grid (Fig. 4). We estimated the location of each cluster to the nearest Io' x Io' cell of the Minor Grid. We then recorded every cluster's Major and Minor Grid location, butterfly count estimate, tree identifier, and height on our data sheets. 


\section{Pismo Butterfly Major Grid Index}

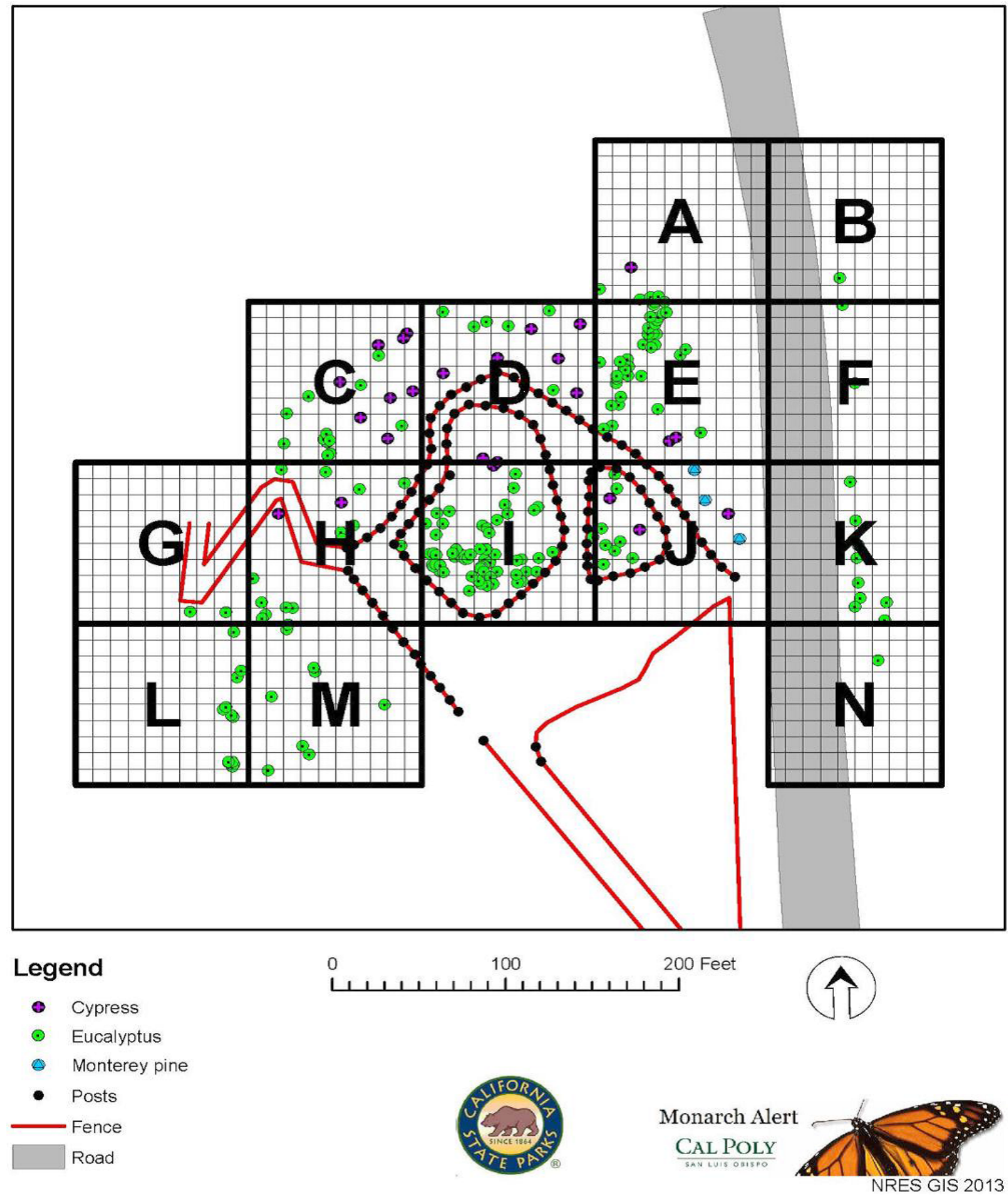

Figure 3. Major Grid 


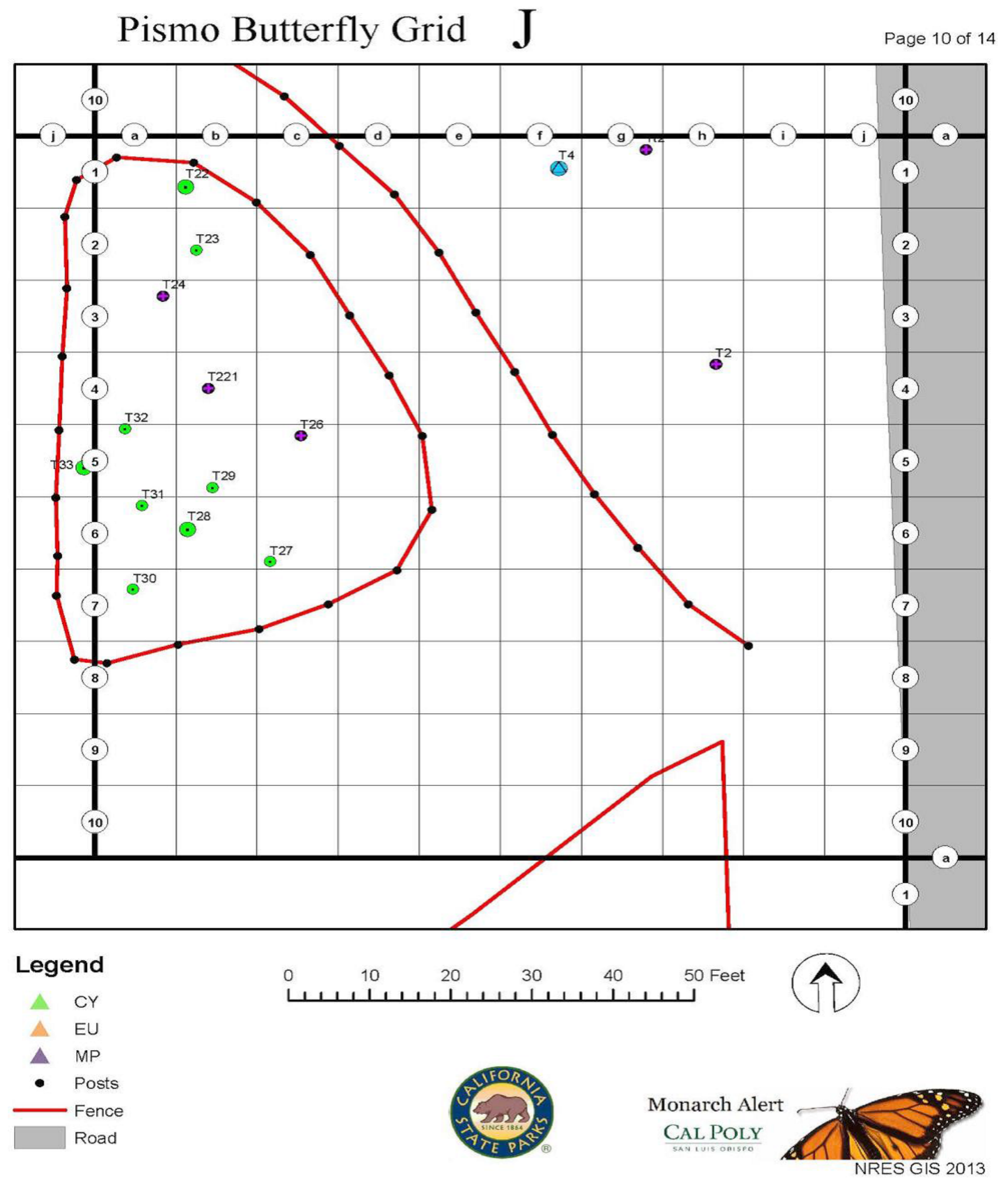

Figure 4. Minor Grid 
Collected data was entered into one Excel spreadsheet, saved as a .csv file type, and imported into ArcMap. The Major and Minor Grids, roads, and fences created in ArcMap by the previous student research groups were reused and added in as base layers. We then used several tools available in ArcMap to display butterfly density redistribution.

The "Table to Table" tool was used to convert the .csv file into a .dbf file (Fig 5). This file type can be added into ArcMap as an attribute table (Fig. 6).

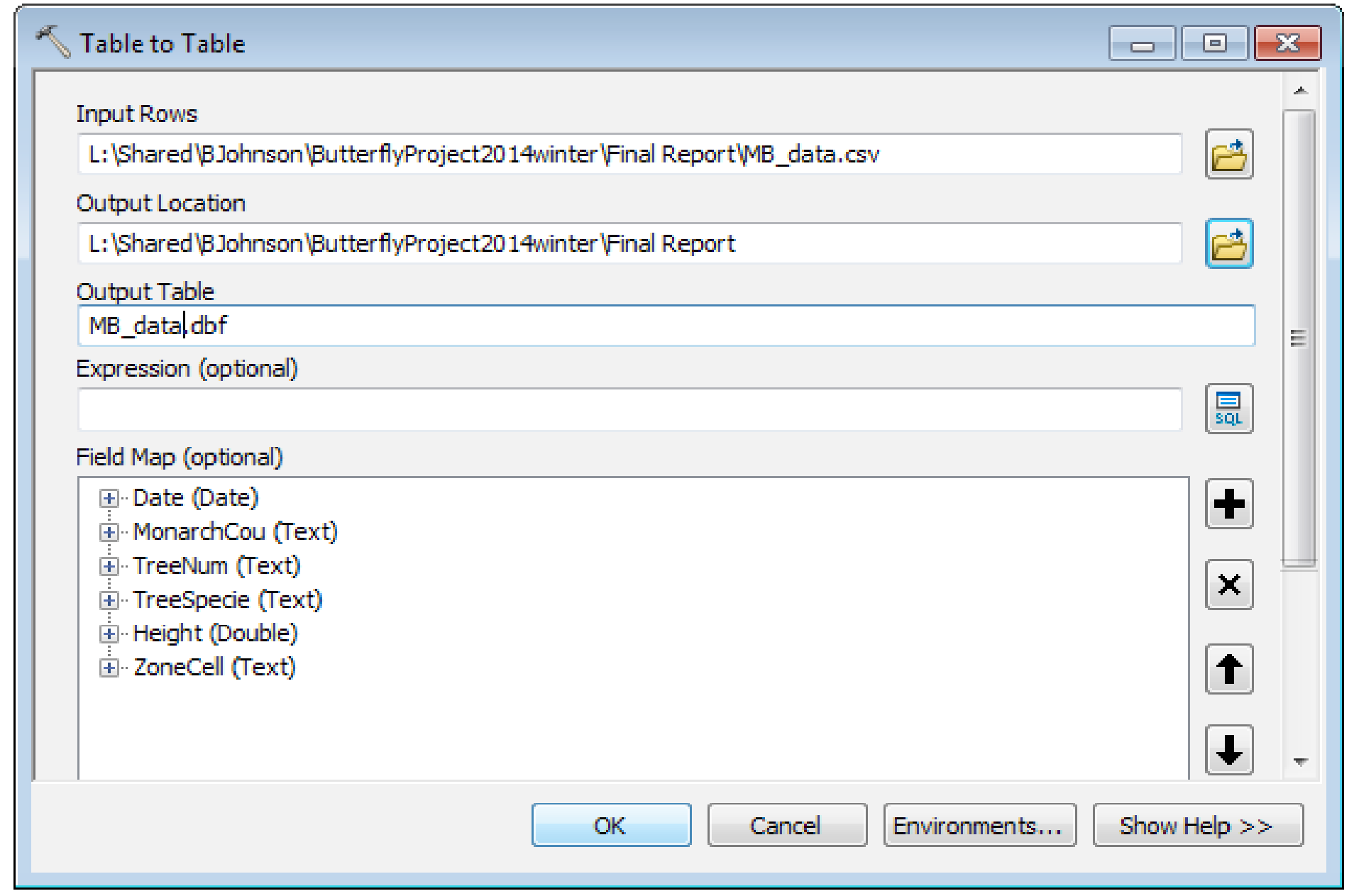

Figure 5. "Table to Table" tool 


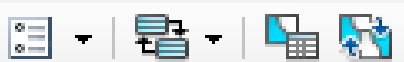

\section{MB_data}

\begin{tabular}{|c|c|c|c|c|c|c|c|}
\hline & OID & Date & MonarchCou & TreeNum & TreeSpecie & Height & ZoneCell \\
\hline • & 0 & $11 / 1 / 2014$ & 12 & $T 63$ & $\bar{E}$ & 3 & $\mathrm{D}-\mathrm{i}-1$ \\
\hline & 1 & $11 / 1 / 2014$ & 45 & T63 & $\mathrm{E}$ & 3 & $\mathrm{D}-\mathrm{i}-1$ \\
\hline & 2 & $11 / 1 / 2014$ & 15 & T63 & $\mathrm{E}$ & 3 & $\mathrm{D}-\mathrm{i}-1$ \\
\hline & 3 & $11 / 1 / 2014$ & 22 & T63 & $\mathrm{E}$ & 4 & D-h-1 \\
\hline & 4 & $11 / 1 / 2014$ & 45 & T63 & $\mathrm{E}$ & 4 & D-h-1 \\
\hline & 5 & $11 / 1 / 2014$ & 15 & T63 & $\mathrm{E}$ & 4 & D-h-1 \\
\hline & 6 & $11 / 1 / 2014$ & 55 & T63 & $\mathrm{E}$ & 4 & D-h-1 \\
\hline & 7 & $11 / 1 / 2014$ & 36 & T63 & $\mathrm{E}$ & 4 & D-h-1 \\
\hline & 8 & $11 / 1 / 2014$ & 28 & T69 & $\mathrm{E}$ & 10 & D-e-3 \\
\hline & 9 & $11 / 1 / 2014$ & 1200 & $\mathrm{~T} 70$ & $\mathrm{E}$ & 10.5 & D-d-4 \\
\hline & 10 & $11 / 1 / 2014$ & 48 & $T 70$ & $\mathrm{E}$ & 10 & D-d-4 \\
\hline & 11 & $11 / 1 / 2014$ & 32 & $\mathrm{~T} 70$ & $\mathrm{E}$ & 11 & D-d-4 \\
\hline & 12 & $11 / 1 / 2014$ & 18 & $\mathrm{~T} 70$ & $\mathrm{E}$ & 10 & D-d-4 \\
\hline & 13 & $11 / 1 / 2014$ & 11 & $T 70$ & $\mathrm{E}$ & 10 & D-d-4 \\
\hline & 14 & $11 / 1 / 2014$ & 41 & $\mathrm{~T} 70$ & $\mathrm{E}$ & 11 & $D-d-4$ \\
\hline & 15 & $11 / 1 / 2014$ & 140 & $T 70$ & $\mathrm{E}$ & 11 & D-d-4 \\
\hline & 16 & $11 / 1 / 2014$ & 260 & $\mathrm{~T} 70$ & $\mathrm{E}$ & 10 & $D-c-4$ \\
\hline & 17 & $11 / 1 / 2014$ & 55 & $\mathrm{~T} 70$ & $\mathrm{E}$ & 10 & $D-c-4$ \\
\hline & 18 & $11 / 1 / 2014$ & 28 & $\mathrm{~T} 70$ & $\mathrm{E}$ & 10 & $D-c-4$ \\
\hline & 19 & $11 / 1 / 2014$ & 30 & $\mathrm{~T} 70$ & $\mathrm{E}$ & 10 & $D-c-4$ \\
\hline & 20 & $11 / 1 / 2014$ & 65 & T81 & $\mathrm{E}$ & 7 & C-i-8 \\
\hline & 21 & $11 / 1 / 2014$ & 14 & T81 & $\mathrm{E}$ & 7 & C-i-8 \\
\hline & 22 & $11 / 1 / 2014$ & 40 & $T 81$ & $\mathrm{E}$ & 7 & C-i-8 \\
\hline & 23 & $11 / 1 / 2014$ & 15 & T81 & $\mathrm{E}$ & 7 & C-i-8 \\
\hline & 24 & $11 / 1 / 2014$ & 18 & T81 & $\mathrm{E}$ & 7 & C-i-8 \\
\hline & 25 & $11 / 1 / 2014$ & 30 & T81 & $\mathrm{E}$ & 7 & C-i-8 \\
\hline & 26 & $11 / 1 / 2014$ & 30 & T81 & $\mathrm{E}$ & 7 & C-i-9 \\
\hline & 27 & $11 / 1 / 2014$ & 110 & T94 & $\mathrm{E}$ & 8 & C-h-8 \\
\hline & 28 & $11 / 1 / 2014$ & 80 & T94 & $\mathrm{E}$ & 8 & C-h-8 \\
\hline & 29 & $11 / 1 / 2014$ & 12 & T94 & $\mathrm{E}$ & 8 & C-h-8 \\
\hline & 30 & $11 / 1 / 2014$ & 110 & T100 & C & 8 & D-b-10 \\
\hline & 31 & $11 / 1 / 2014$ & 35 & T100 & C & 8 & D-b-10 \\
\hline & 32 & $11 / 1 / 2014$ & 12 & T100 & C & 8 & D-b-10 \\
\hline & 33 & $11 / 1 / 2014$ & 18 & T100 & C & 8 & D-b-10 \\
\hline & 34 & $11 / 1 / 2014$ & 330 & T100 & C & 12 & D-c-9 \\
\hline & 35 & $11 / 1 / 2014$ & 210 & T100 & C & 12 & D-c-9 \\
\hline & 36 & $11 / 1 / 2014$ & 45 & T100 & C & 12 & D-c-9 \\
\hline & 37 & $11 / 1 / 2014$ & 95 & T100 & C & 14 & D-d-9 \\
\hline & 38 & $11 / 1 / 2014$ & 22 & T142 & $\mathrm{E}$ & 12 & $1-a-5$ \\
\hline
\end{tabular}

\section{1 ・}

MB data

Figure 6. Attribute Table 
The "Create Address Locator" tool was used to assign each unique Io' x Io' cell in the Minor Grid to a field labeled "ZoneCell" (Fig. 7). Our Minor Grid layer was used as the primary table and the attribute field "MinorGridLabel" was used as the Key Field.

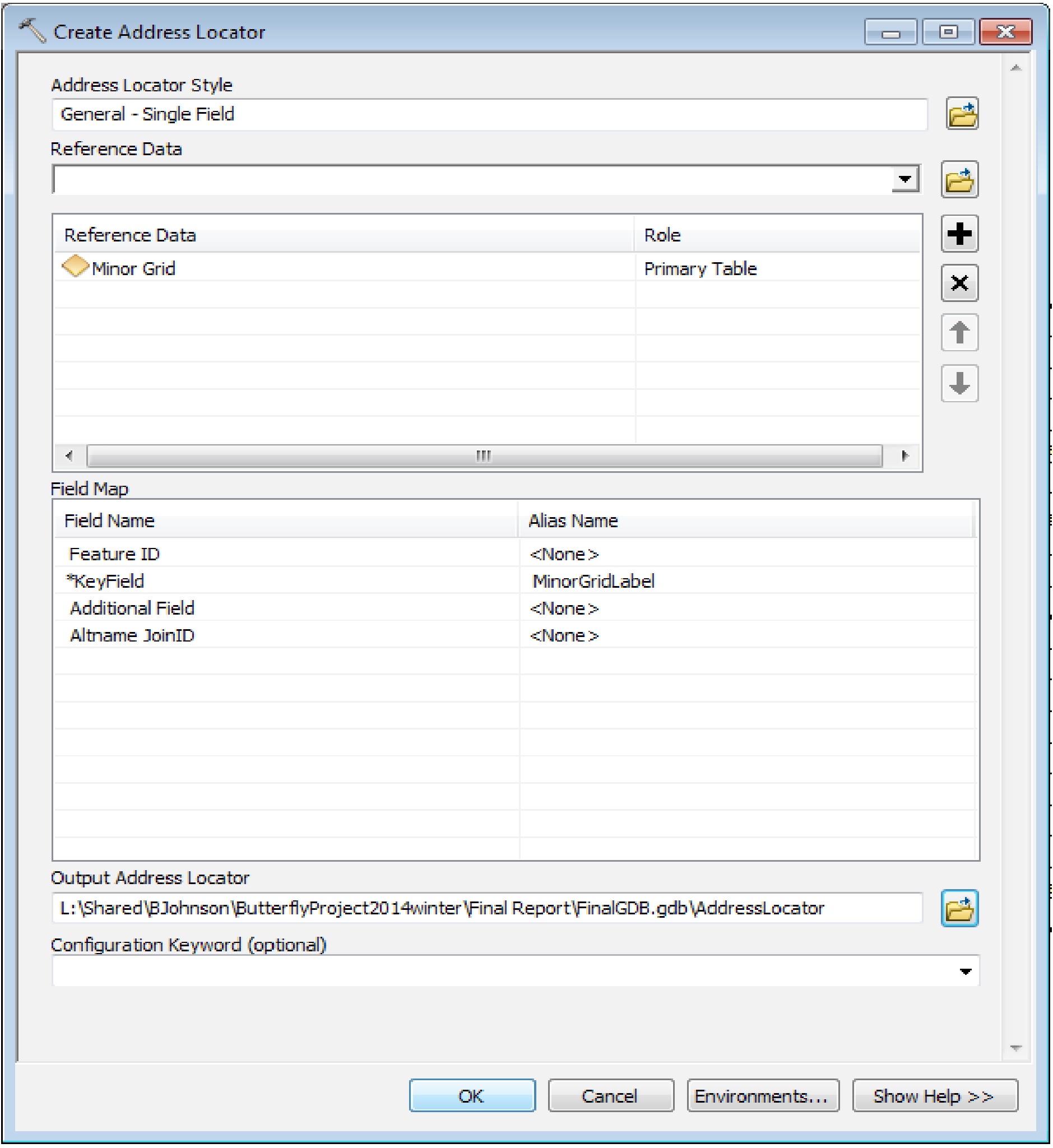

Figure 7: "Create Address Locator" tool 
We then geocoded the addresses assigned to each individual cluster to the newly created addresses using the "Geocode Addresses" tool (Fig 8). This process linked the recorded "ZoneCell" data to the addresses on the Minor Grid. We set geocoding options for spelling sensitivity to be Ioo\% (Fig. 9). This resulted in IO०\% matched addresses (Fig. IO).

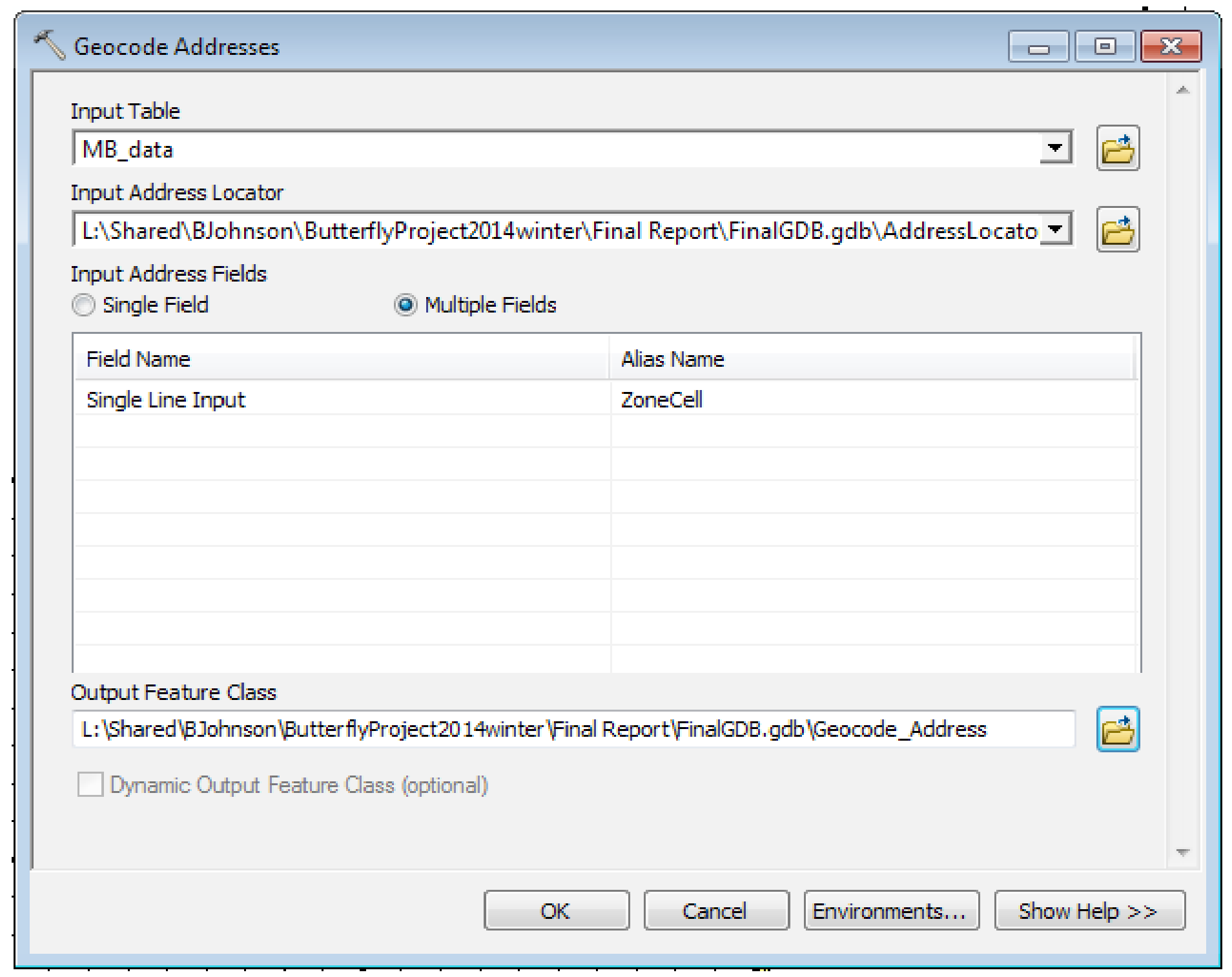

Figure 8: "Geocode Addresses" tool 
Geocoding Options

Matching Options

Place Name Alias Table...

Spelling sensitivity:

Minimum candidate score:

Minimum match score:
None $>$

100

10

80

Intersections

Connectors:

Separate connectors by a space, e.g. "8. (I)

Output Options

Side offset:

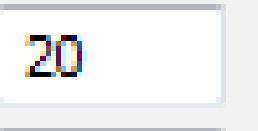

End offset:

3

Feet

Percent

$\checkmark$ Match if candidates tie

Output Fields

$\square \mathrm{X}$ and $\mathrm{Y}$ coordinates

$\nabla$ Reference data ID $\square$ Standardized address

$\square$ Percent along

\section{OK}

Cancel 


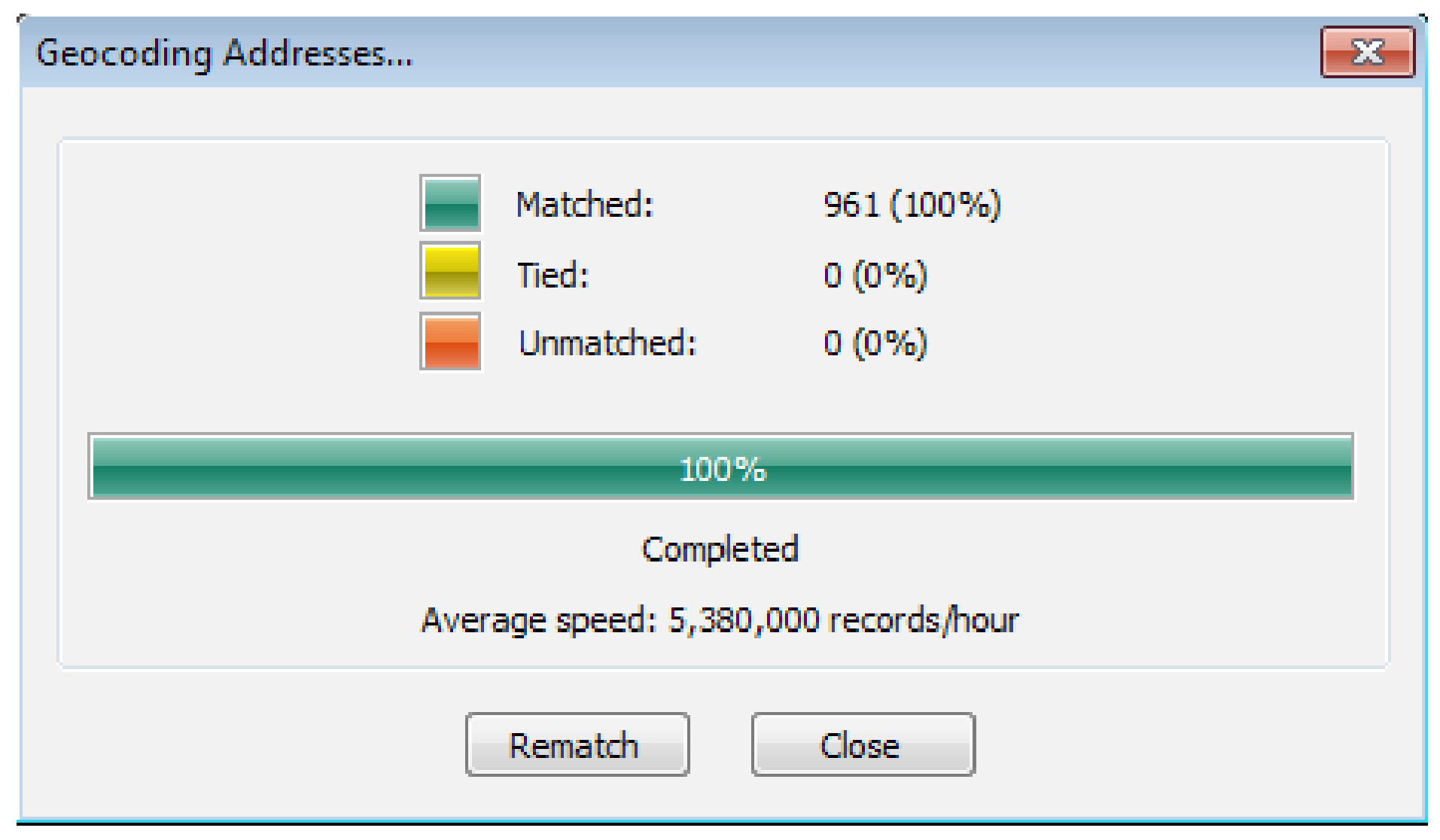

Figure Io: Geocoding Matches 


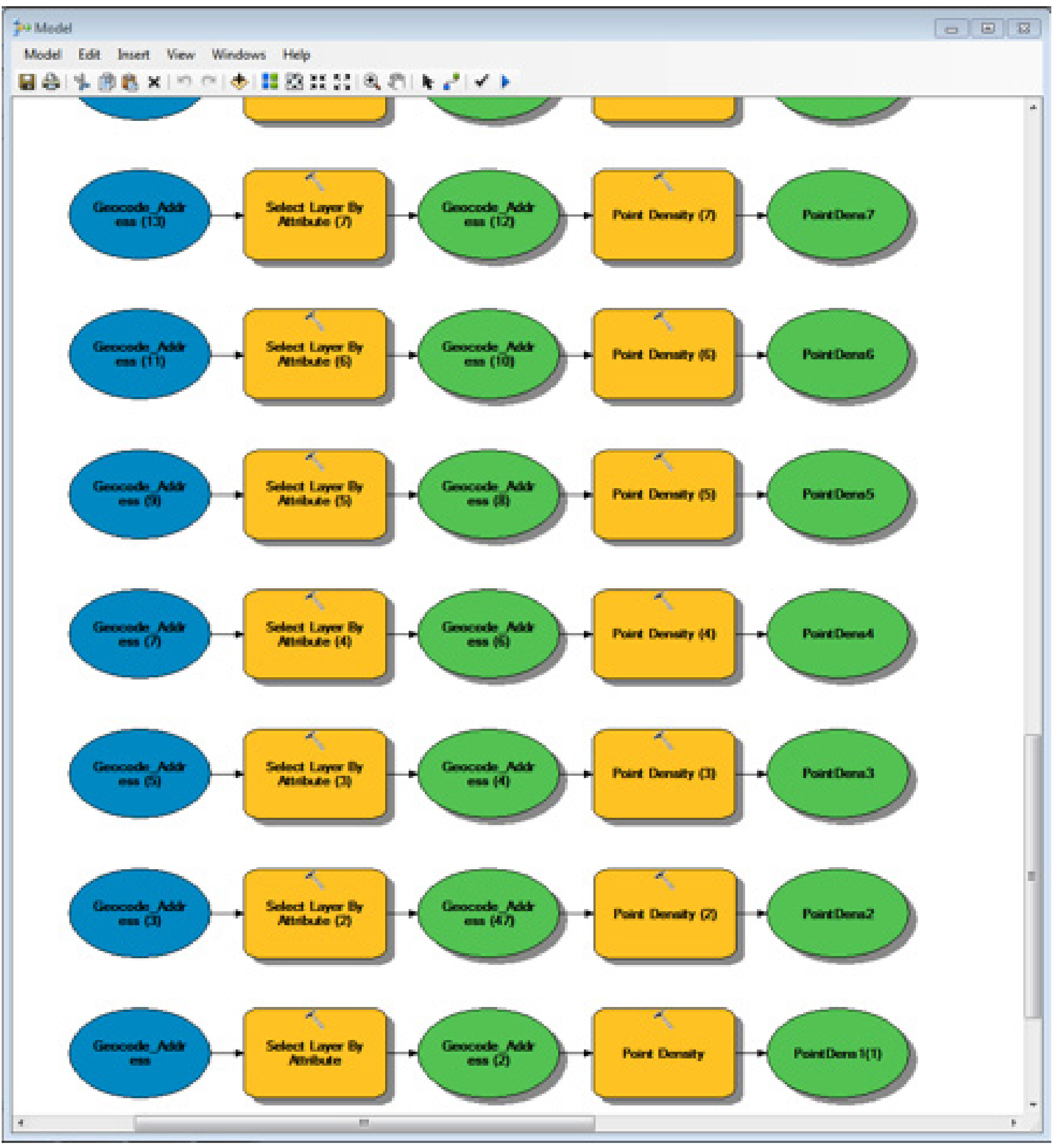

Figure II: Model Builder 
We began creation of the model by adding the "Geocode_Address" layer to our blank model. We then used the "Select Layer by Attribute" tool to select the first date, Nov. I. Then we took the output of this tool and used the "Point Density" tool to display butterfly density in the PMBG on Nov. I. From there, we replicated this model for each of the other twenty-two dates. Then, we went to each line of the model and changed the "Select by Attribute" tool query to select each individual date. We then renamed each of the final outputs to correlate with the date of interest. This model supplied us with twenty-three point density maps — one for each day of our study (Appendix A).

We then created a point density map for the entirety of the study period using the "Point Density" tool (Fig. I2). This yielded the PMBG Point Density Map (Fig. I3).

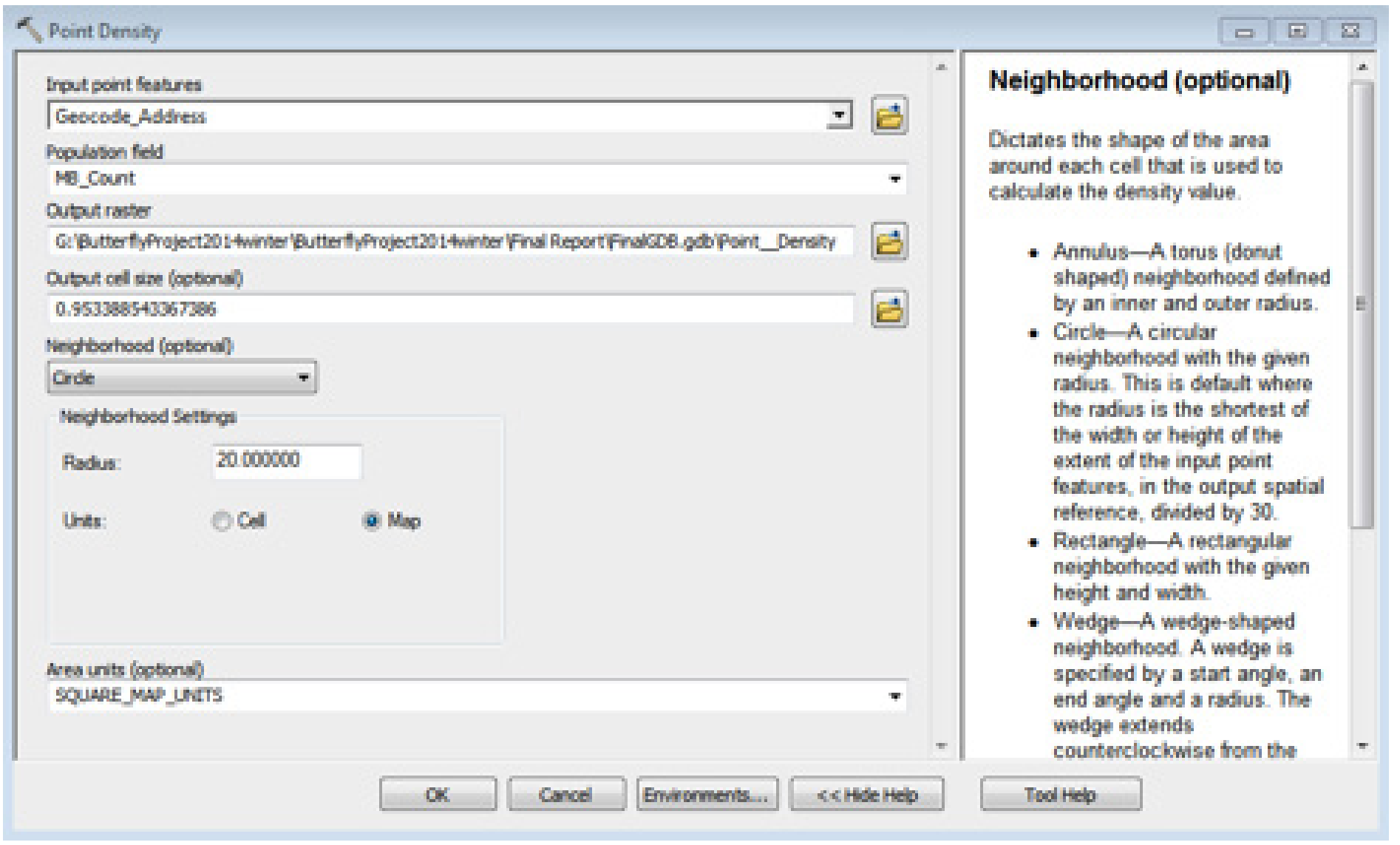

Figure I2: "Point Density" tool 


\section{November 2014 - Pismo Monarch Grove Population Density}

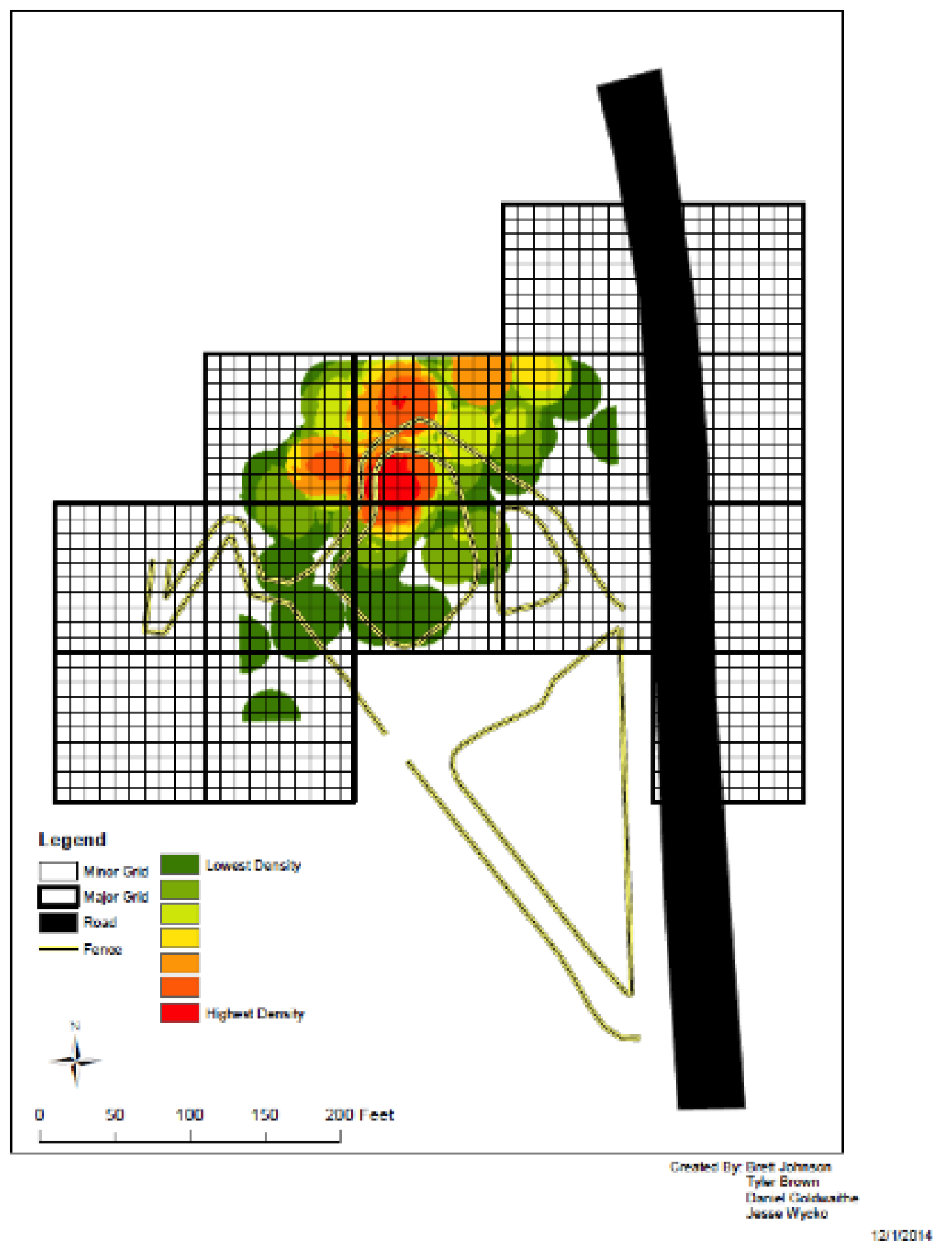

Figure I3: Pismo Beach Monarch Butterfly Grove point density map 
We used the "Feature to 3 D By Attribute" tool to convert our "Height" attribute field into a Z-value useable in ArcScene (Fig. I4).

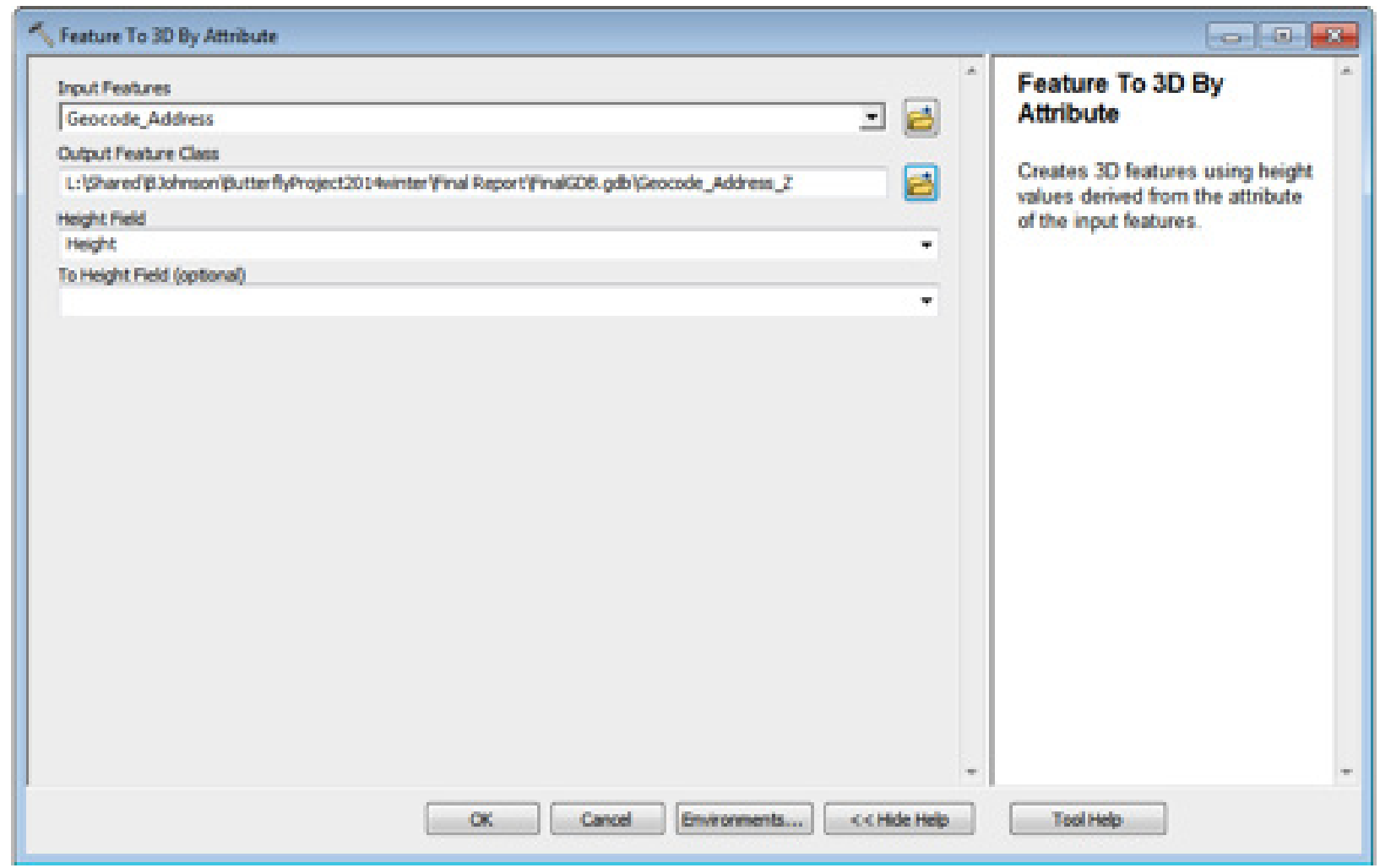

Figure I4: "Feature to 3D By Attribute" tool

We then opened ArcScene I0.2.2 and added in our base layers: the road, fence, and Major/Minor grids (Fig. 15).

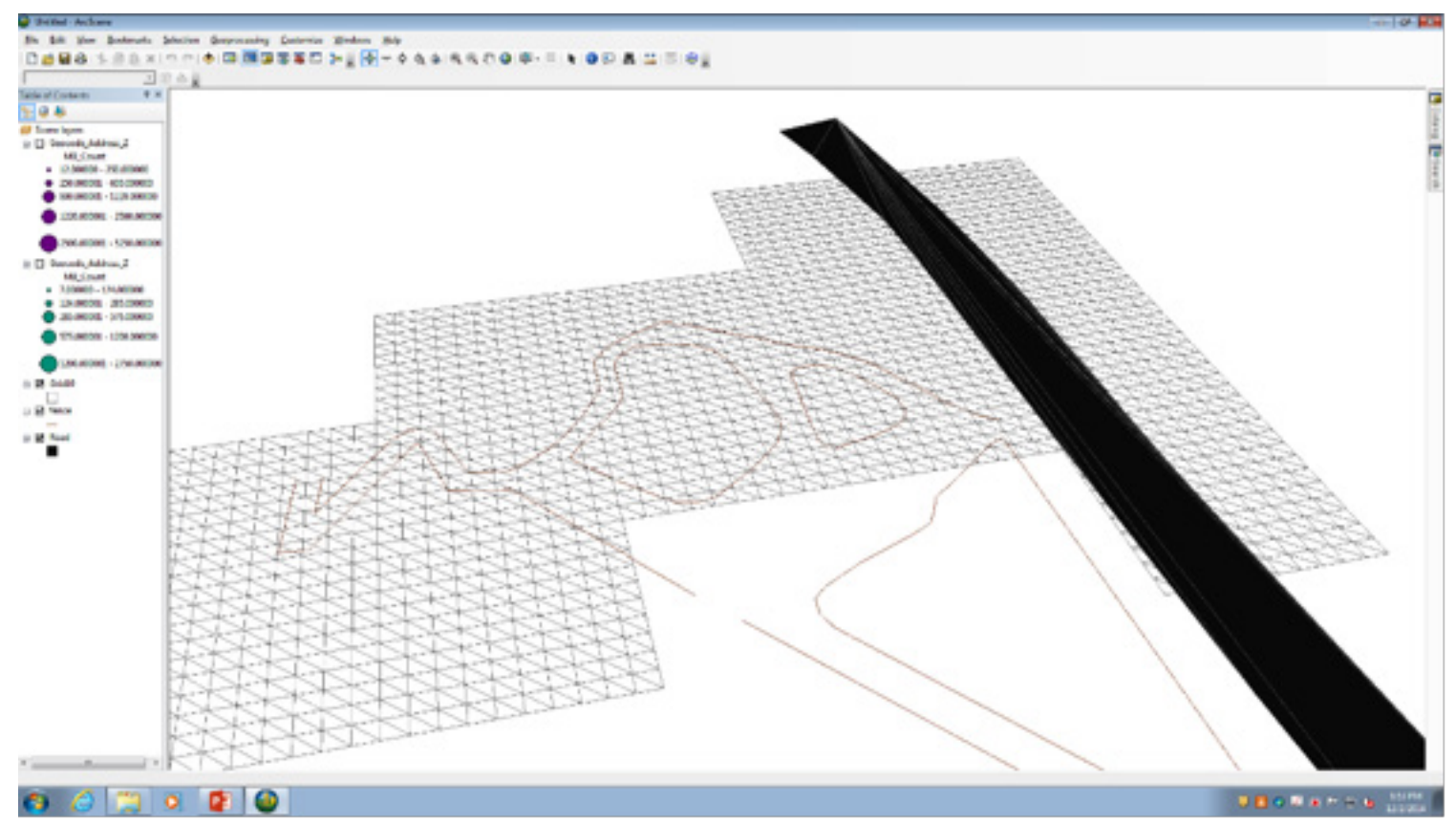

Figure I5: ArcScene Base Layers 
We took the output of the "Feature to 3D by Attribute" tool, a layer named "Geocode_Address_Z," and opened that layer in ArcScene (Fig. I6). We duplicated this layer and used Definition Query (found under properties) to nullify Cypress trees in the first layer and Eucalyptus trees in the second. This allowed us to show Cypress and Eucalyptus trees in the grove at the same time as different colors. We enabled time and elevation from features for both of these layers.

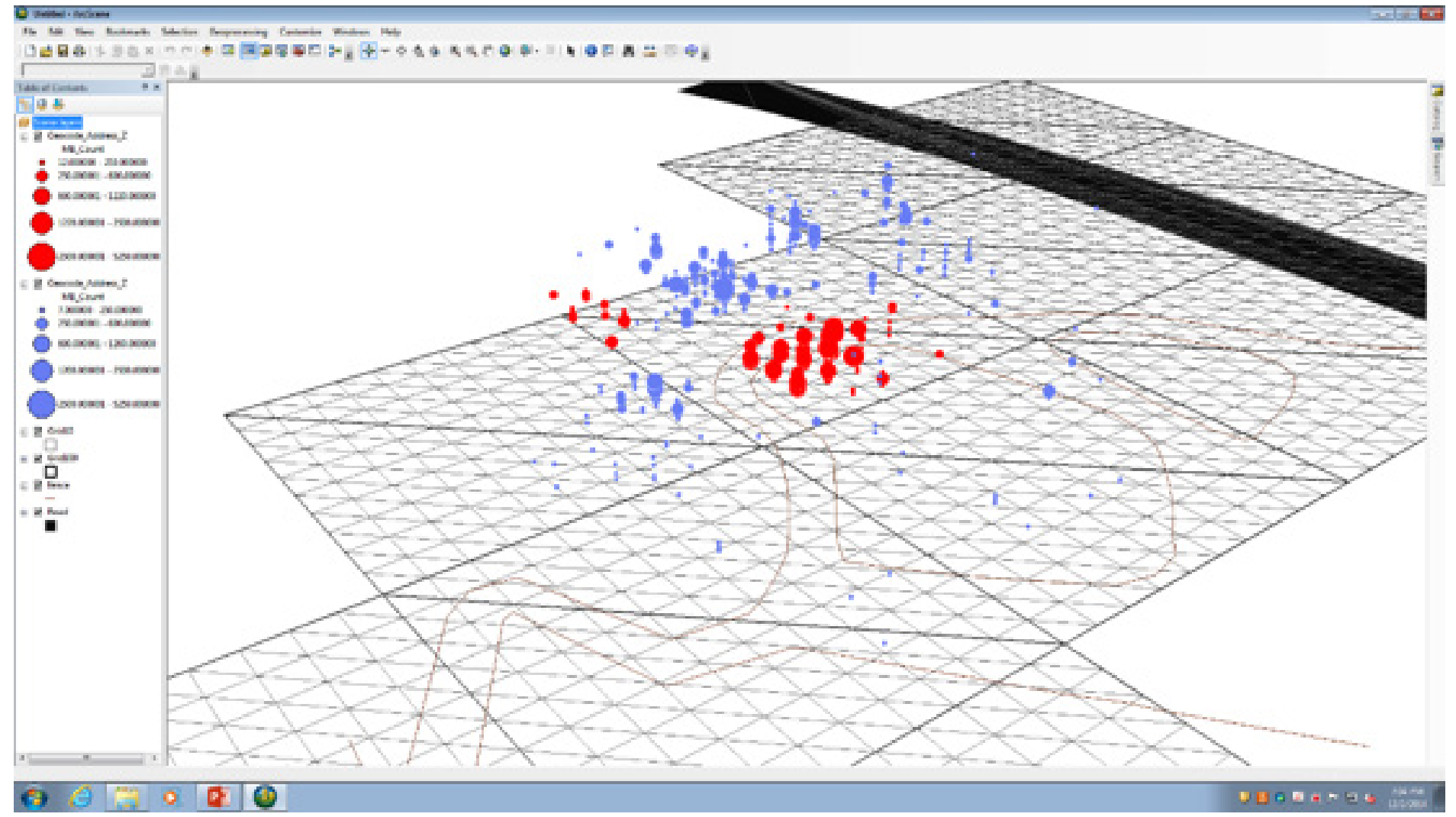

Figure I6: ArcScene Layers 
To create a video animation with time enabled, we opened the Animation Manager and shot several keyframes (Fig. I7). Our animation is available in supplemental materials.

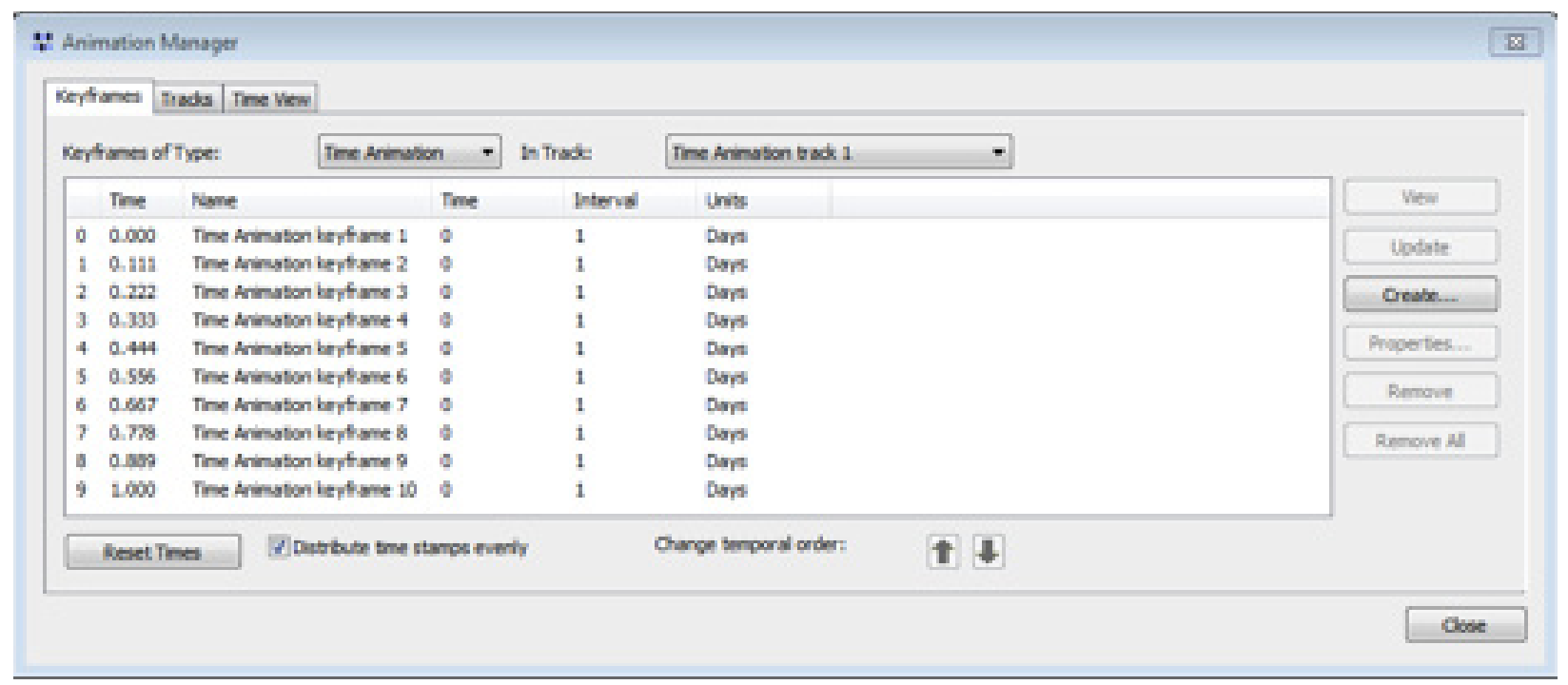

Figure 17: Animation Manager

\section{Results}

Over the course of our study, the number of Monarchs in the grove grew dramatically. On November I, we saw about 3,400 individuals, and on November 23, we saw just over 13,000 individuals. We noticed that clustering on the central Cypress tree became the densest by the end of our study, housing the most Monarchs. We also noticed that many of the clusters moved to greater heights as time went on, which we attribute to decreasing temperatures. Additionally, the butterflies tended to cluster in the center of the grove, leading us to believe that their affinity for the Cypress tree may be a result of its location rather than its physical characteristics. Perhaps the protection of the surrounding Eucalyptus trees is more important to Monarch survival than any benefits of clustering on a Cypress tree. The central location of the Cypress led us to believe that it is the most sheltered from outside winds.

We were able to produce many useable products in both ArcMap and ArcScene. We created a Point Density map for each day of our study and the study period as a whole. We show cluster redistribution from day-to-day in ArcScene, taking into account the height of each cluster. We also provided twentythree days of useful count estimates at the PMBG. 


\section{Acknowledgements}

We would first like to thank Dr. Francis Villablanca of the Cal Poly Biology department for providing inspiration and guidance to both our group and our predecessors. We would also like to thank David Yun for organizing the project and introducing us to the topic, as well as providing valuable guidance in using ArcGIS. We would like to thank Danielle Patterson of California State Parks for training our group in data collection techniques and providing us with specific information on the Pismo Monarch Butterfly Grove. We would like to thank the Cal Poly Wildlife Club for allowing us to present our project to their members. Finally, we would like to thank the following people for committing valuable time and effort into helping us collect the vital field data that made this project possible:
Nicole Durtschi
Jessica Copeland
Hannah Brown
Taylor Steele
Jasmid Rodriguez
Brandi Bergreen
Emily Dektar

Thank you again, everyone. This project would not have been possible without all of your help! 
Appendix A

November 2014 - Pismo Monarch Grove Population Density

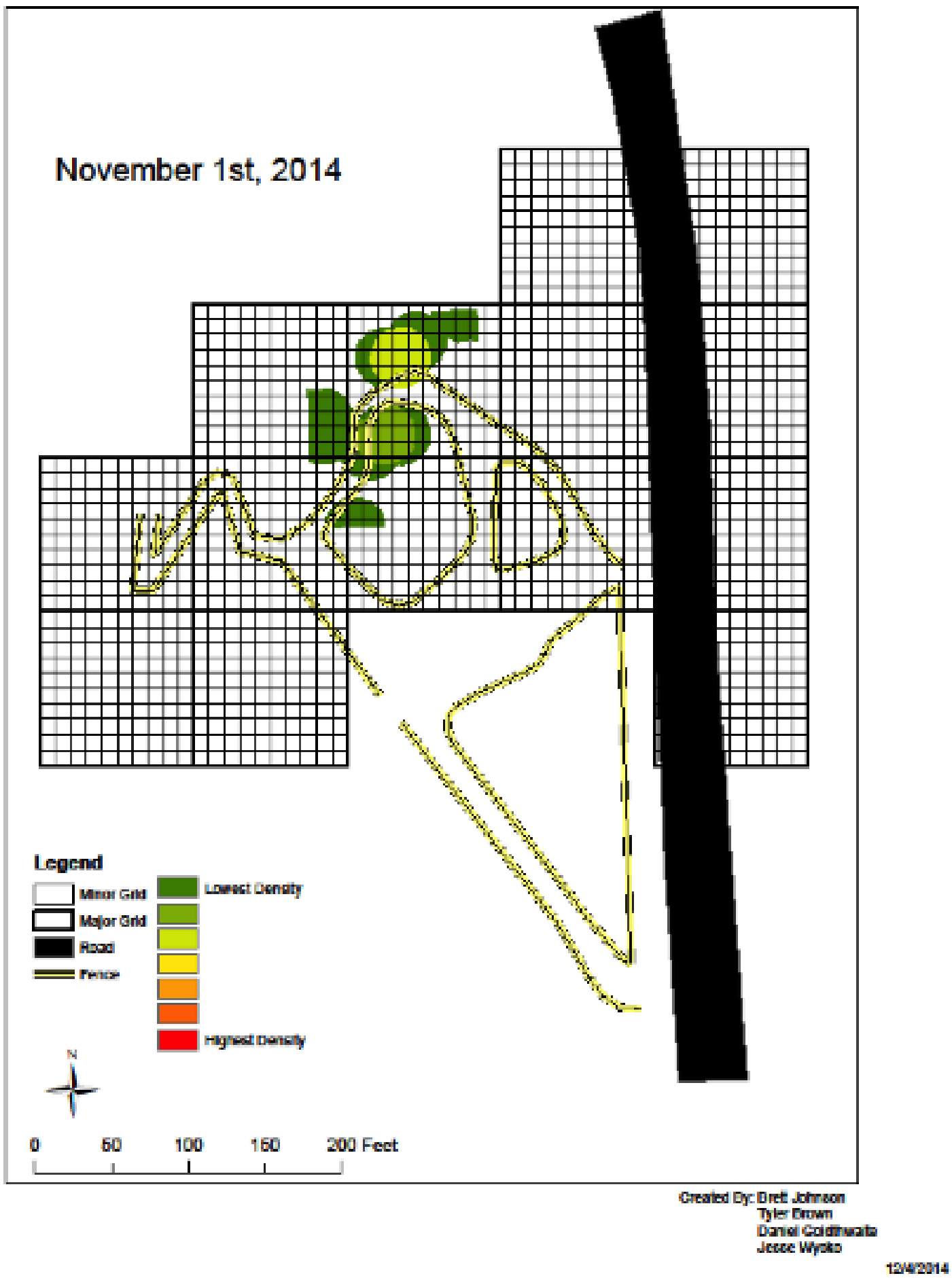




\section{November 2014 - Pismo Monarch Grove Population Density}

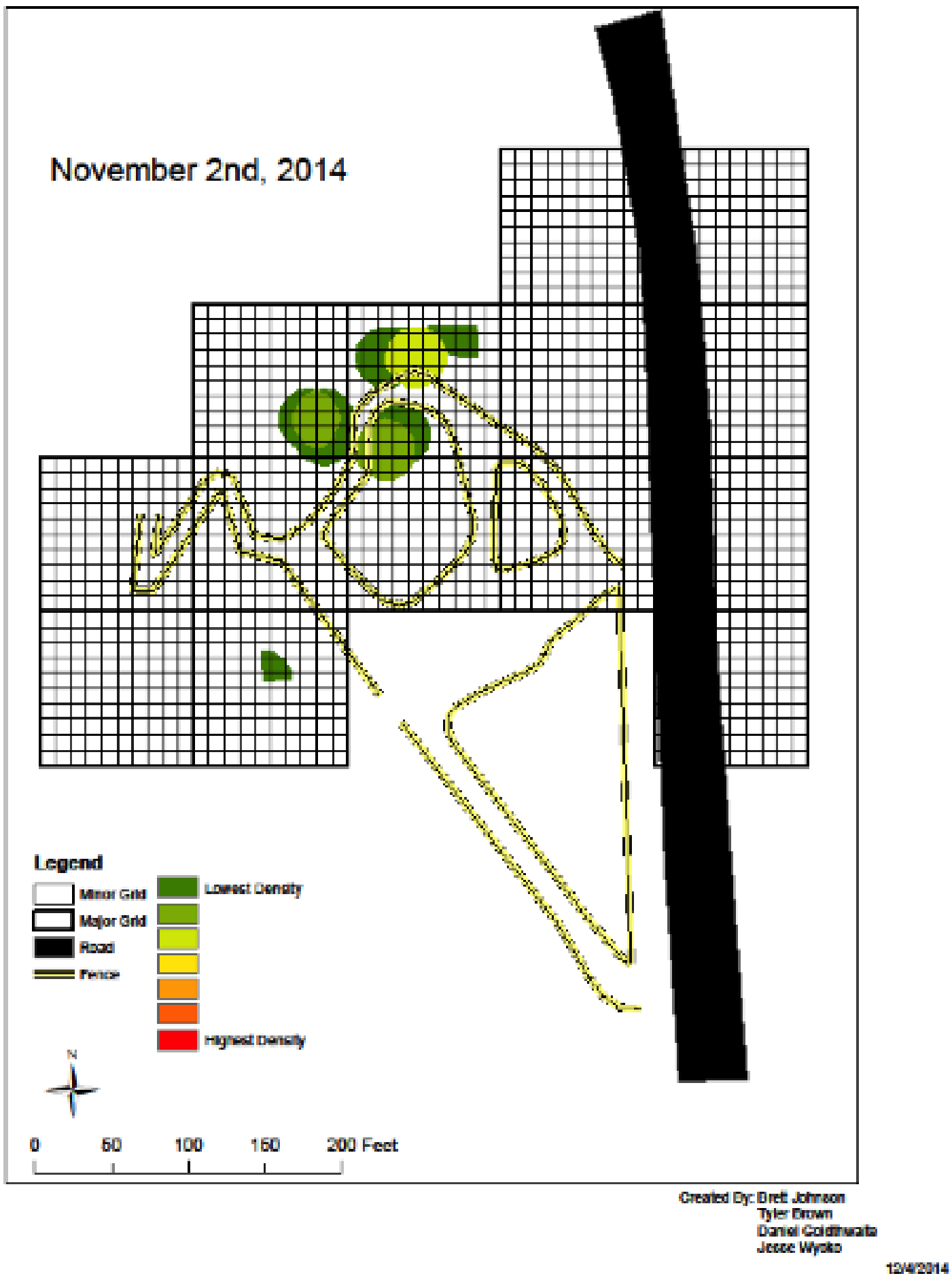




\section{November 2014 - Pismo Monarch Grove Population Density}

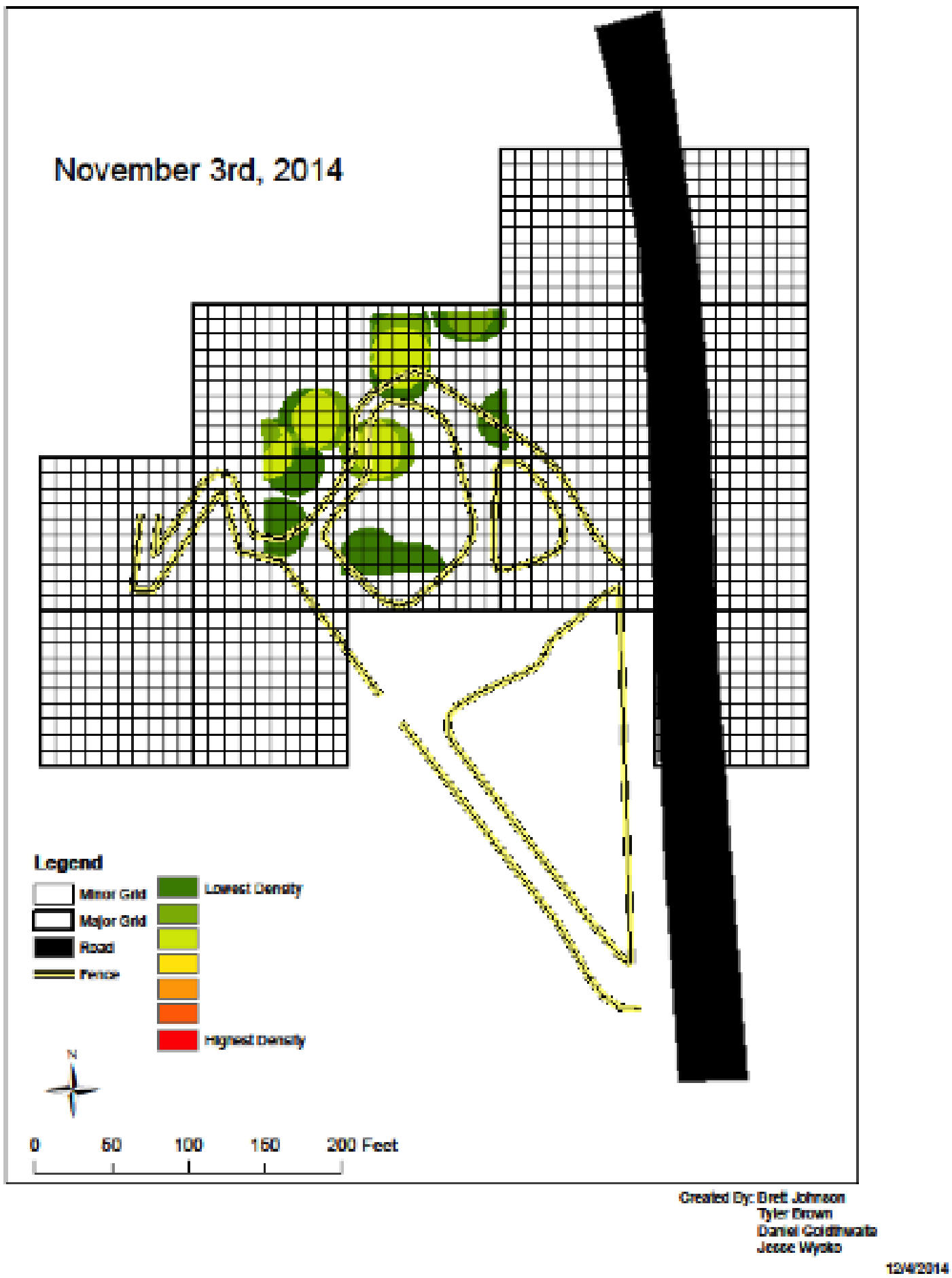




\section{November 2014 - Pismo Monarch Grove Population Density}

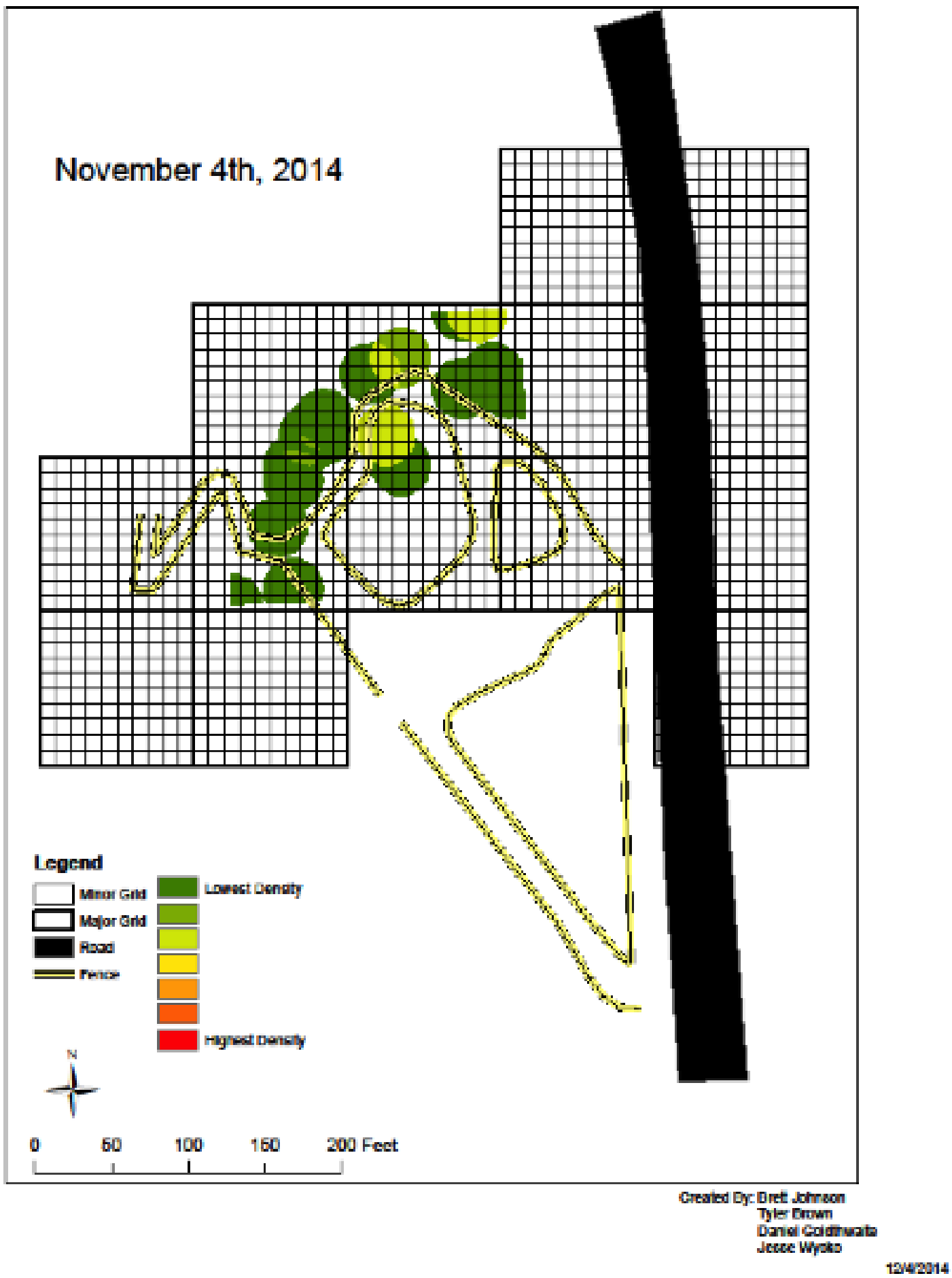


November 2014 - Pismo Monarch Grove Population Density

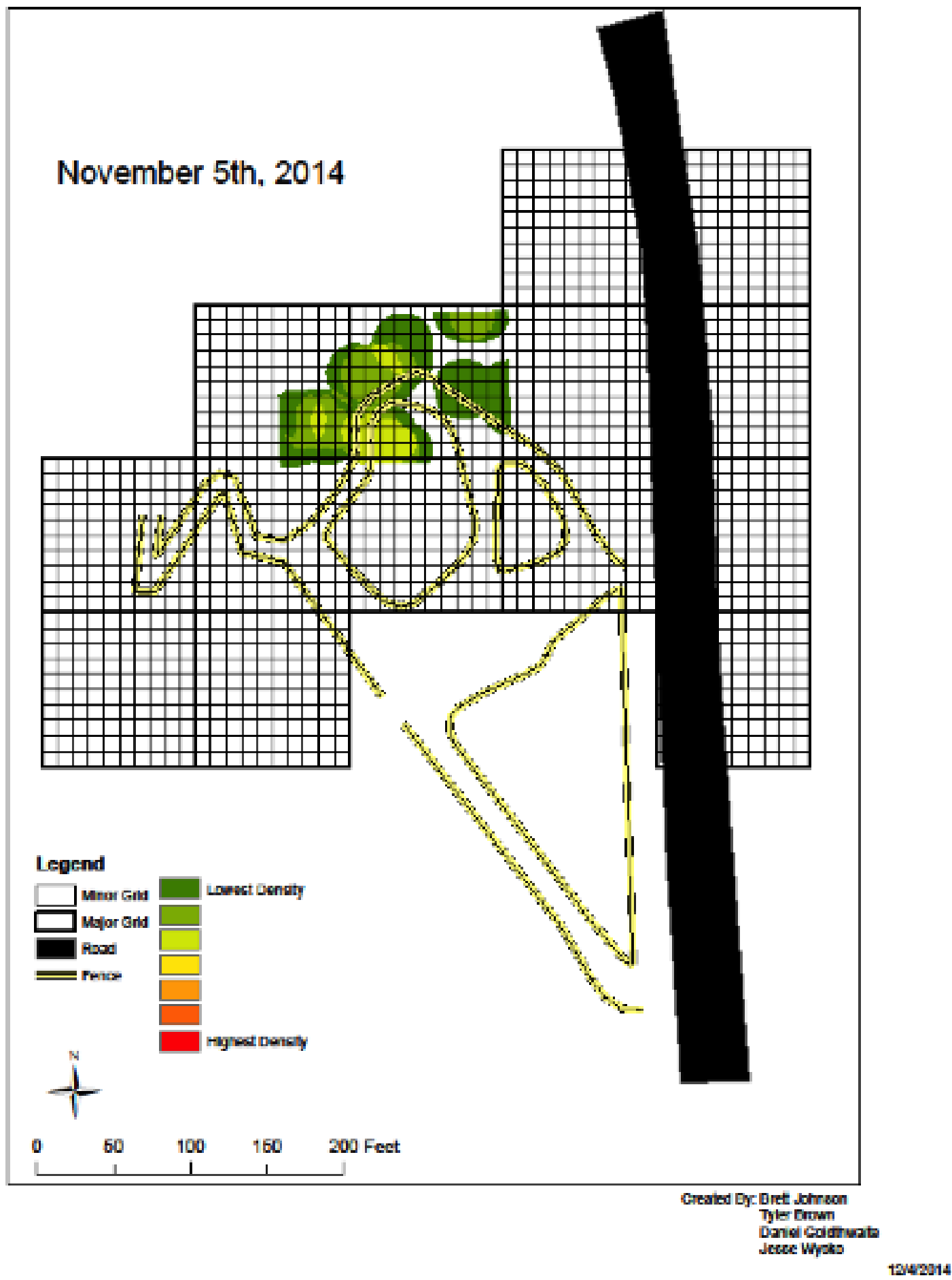


November 2014 - Pismo Monarch Grove Population Density

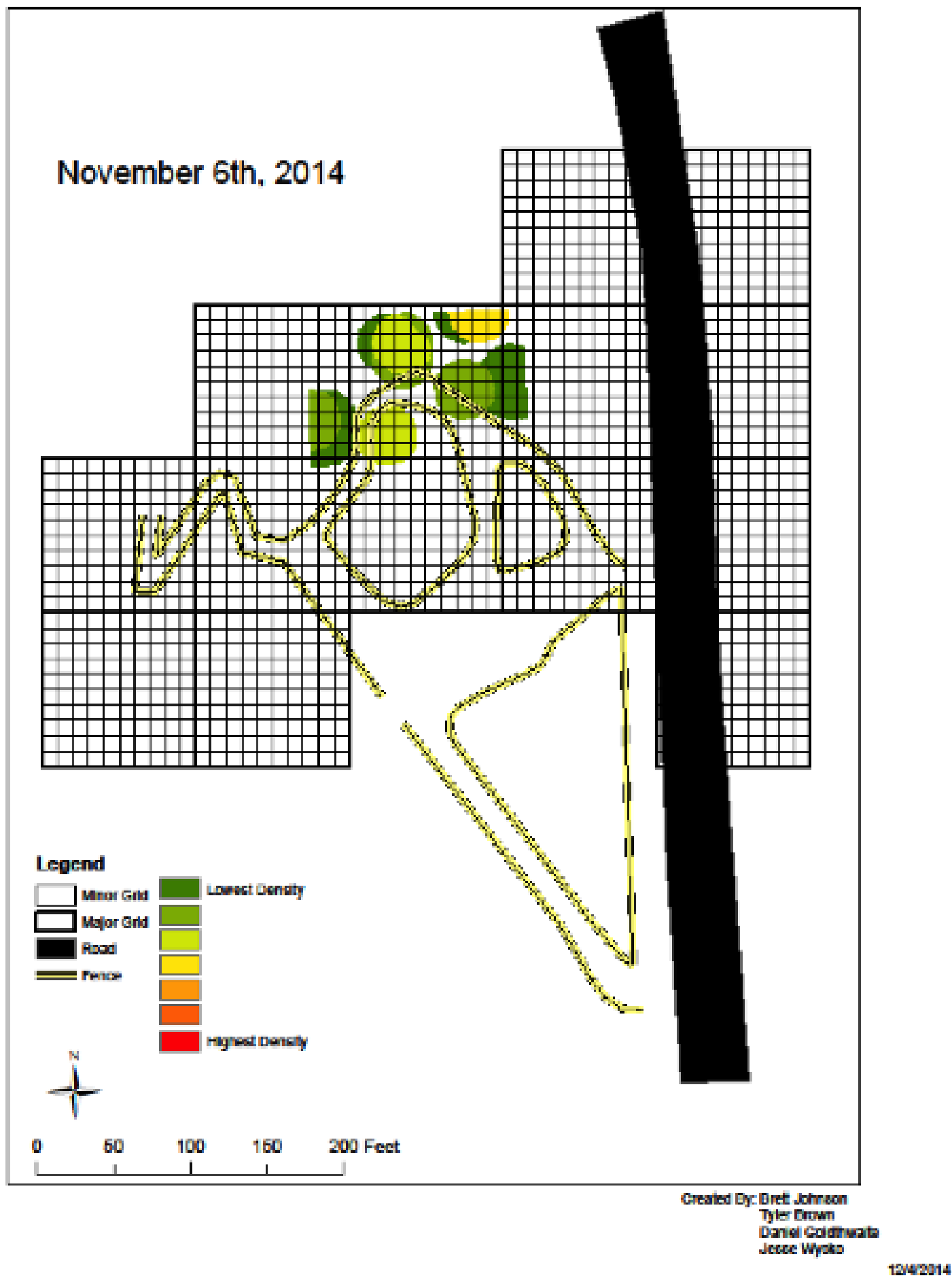


November 2014 - Pismo Monarch Grove Population Density

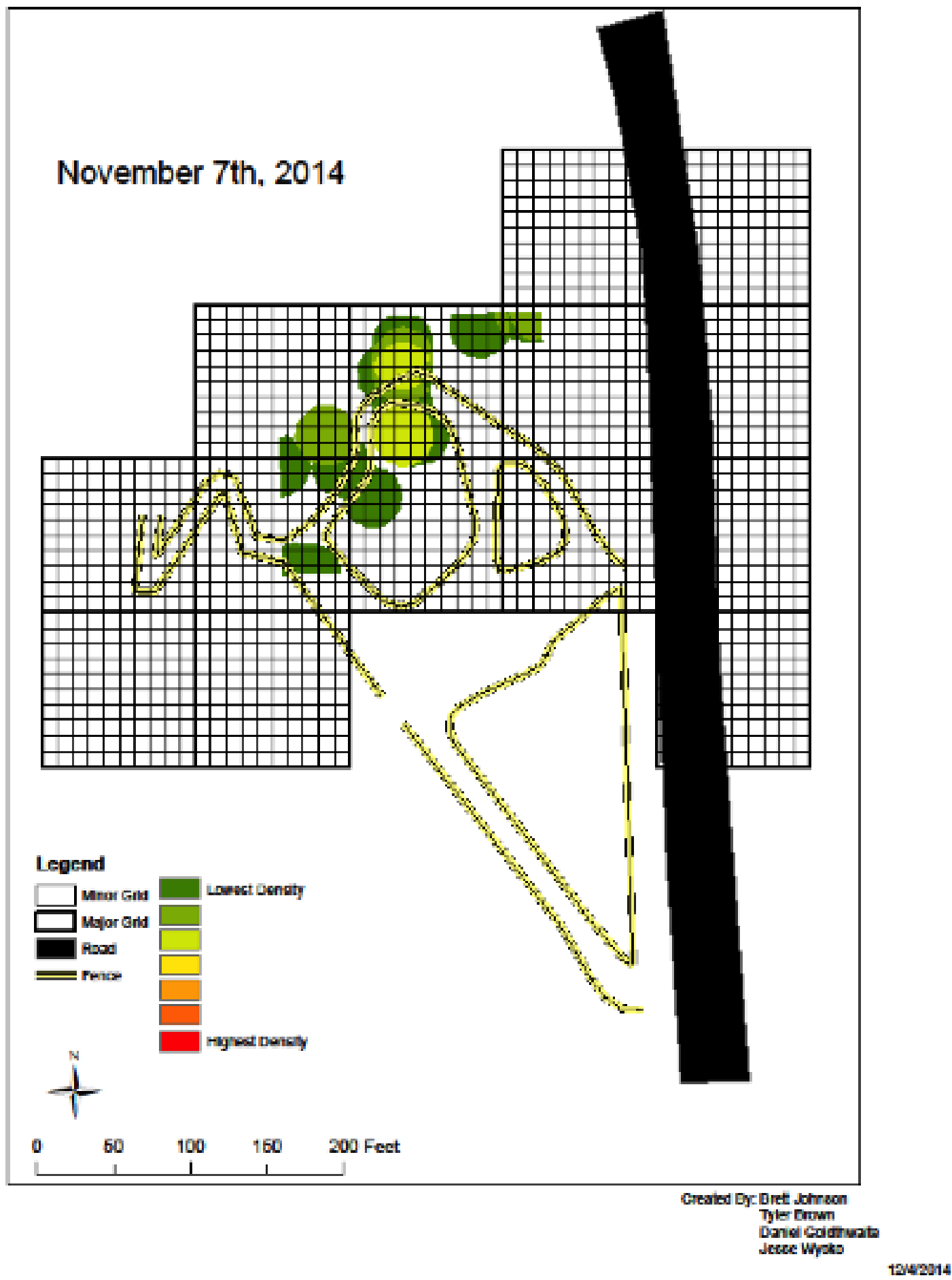


November 2014 - Pismo Monarch Grove Population Density

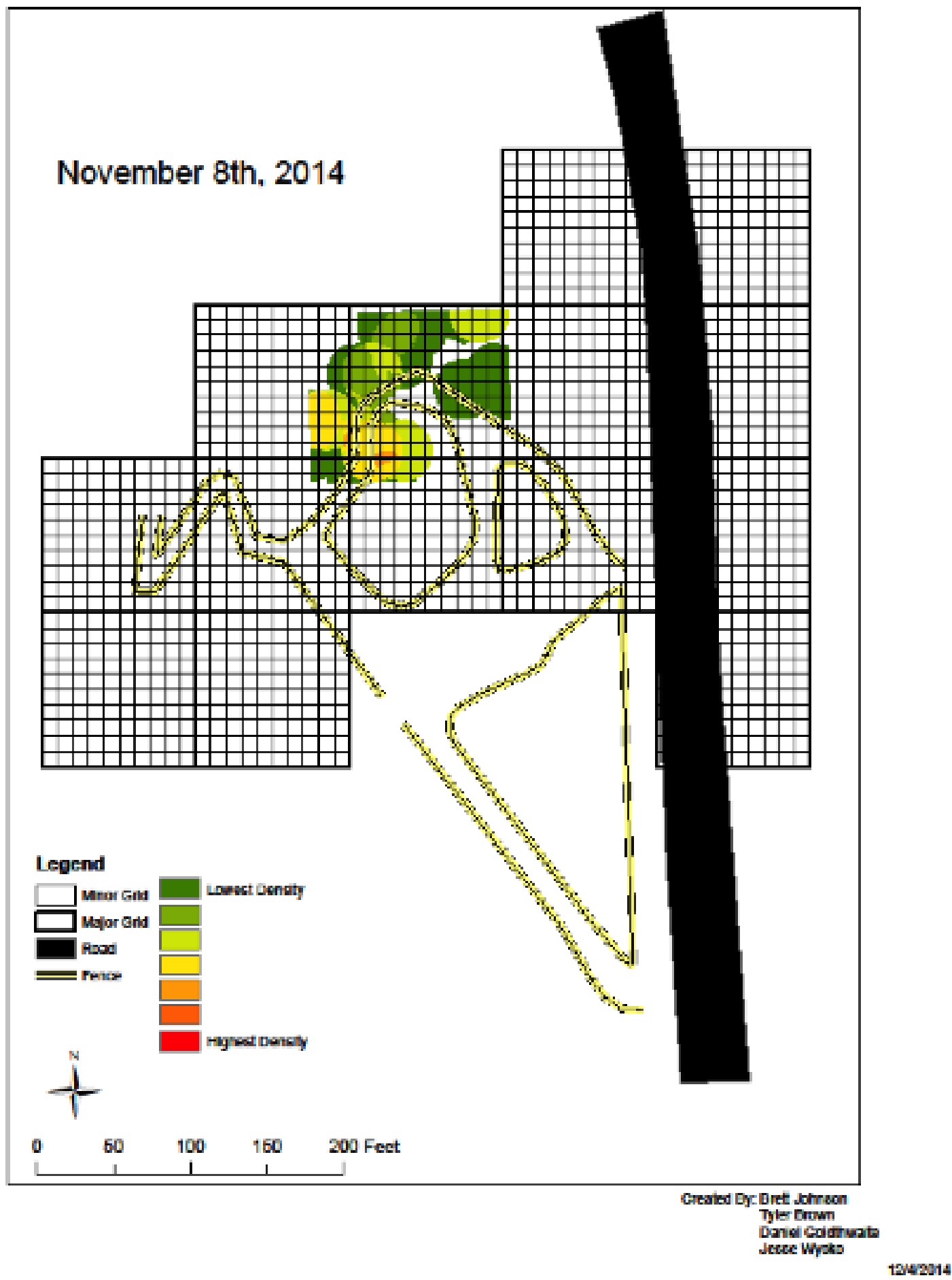


November 2014 - Pismo Monarch Grove Population Density

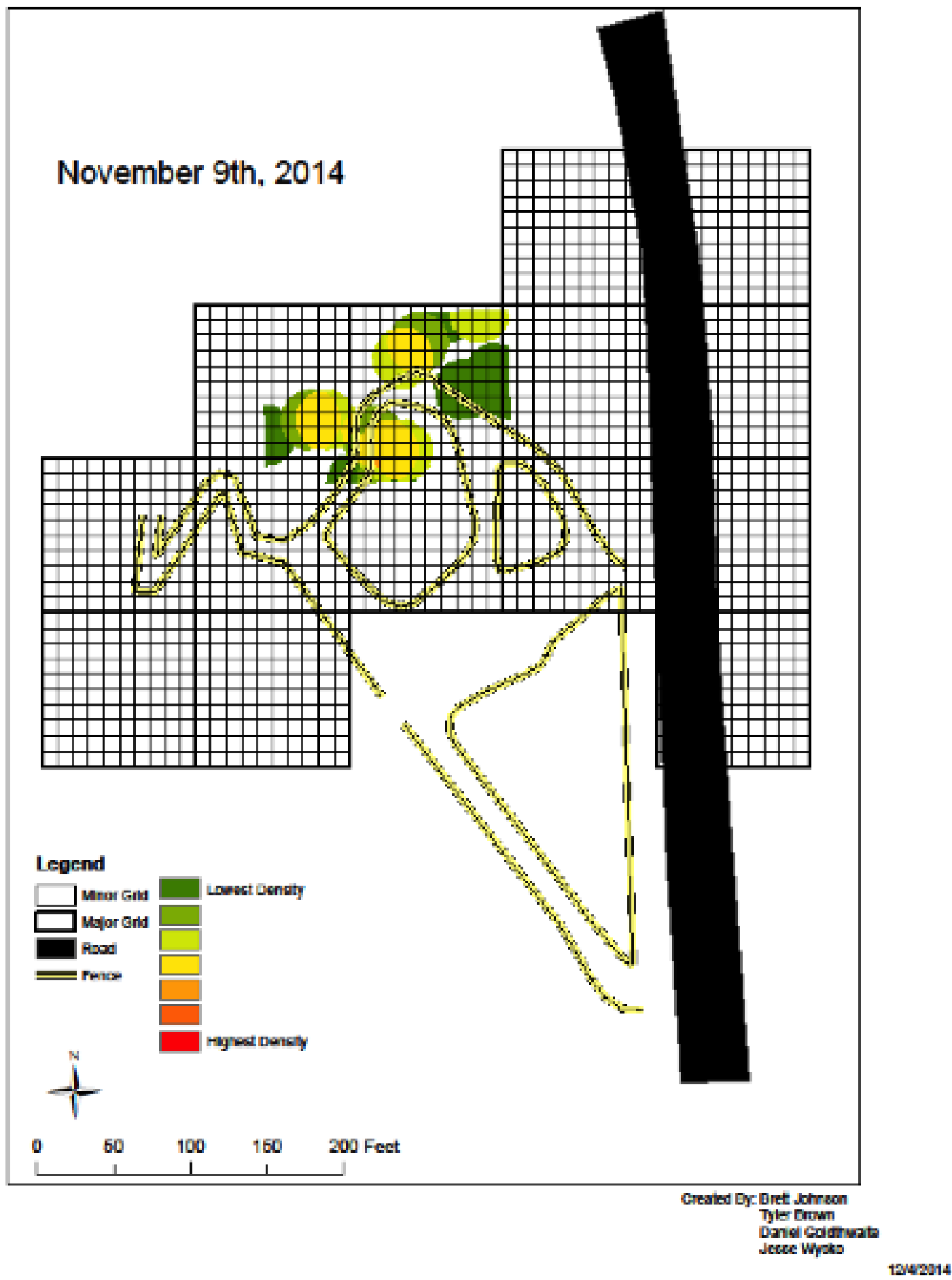


November 2014 - Pismo Monarch Grove Population Density

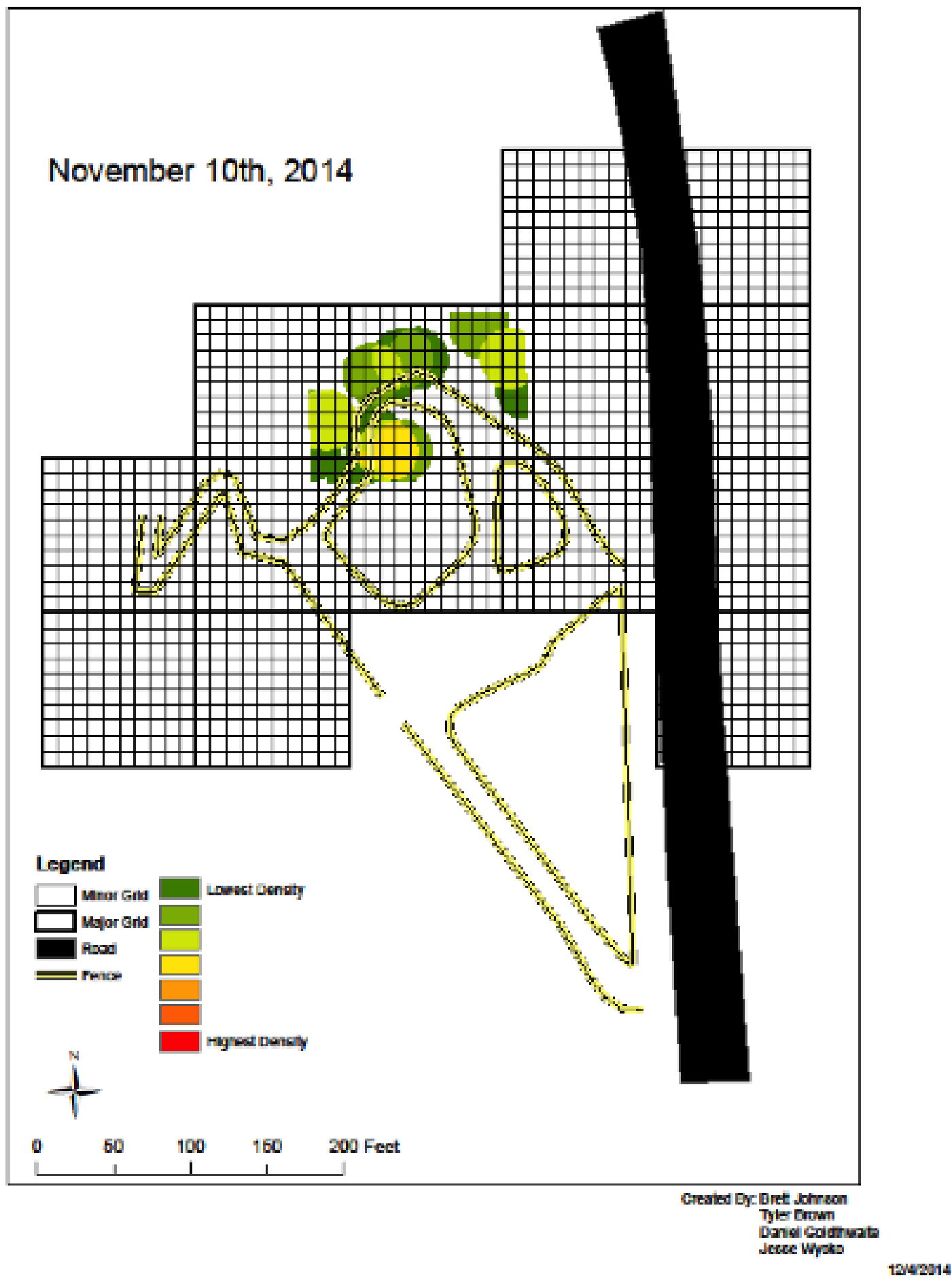




\section{November 2014 - Pismo Monarch Grove Population Density}

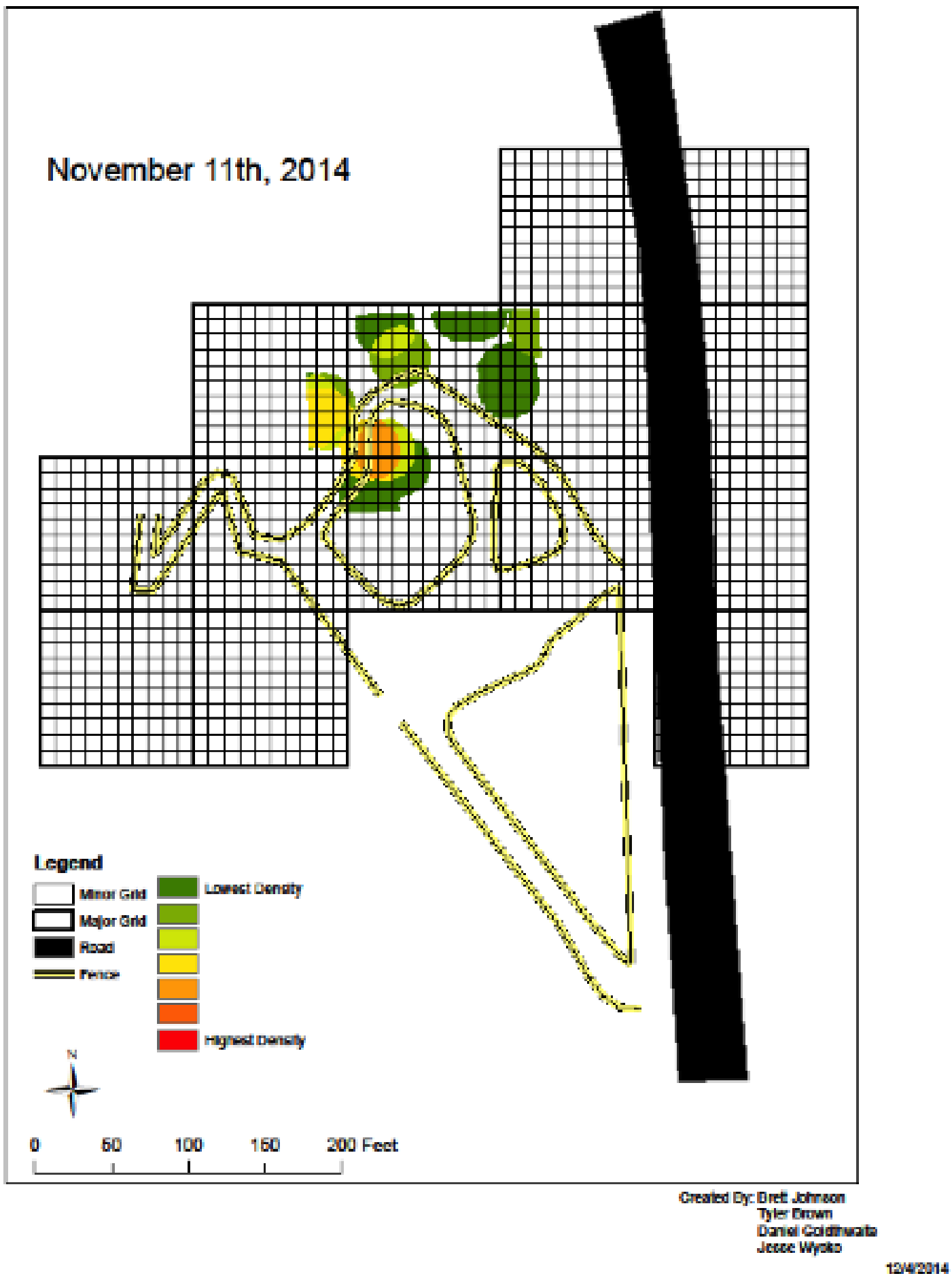


November 2014 - Pismo Monarch Grove Population Density

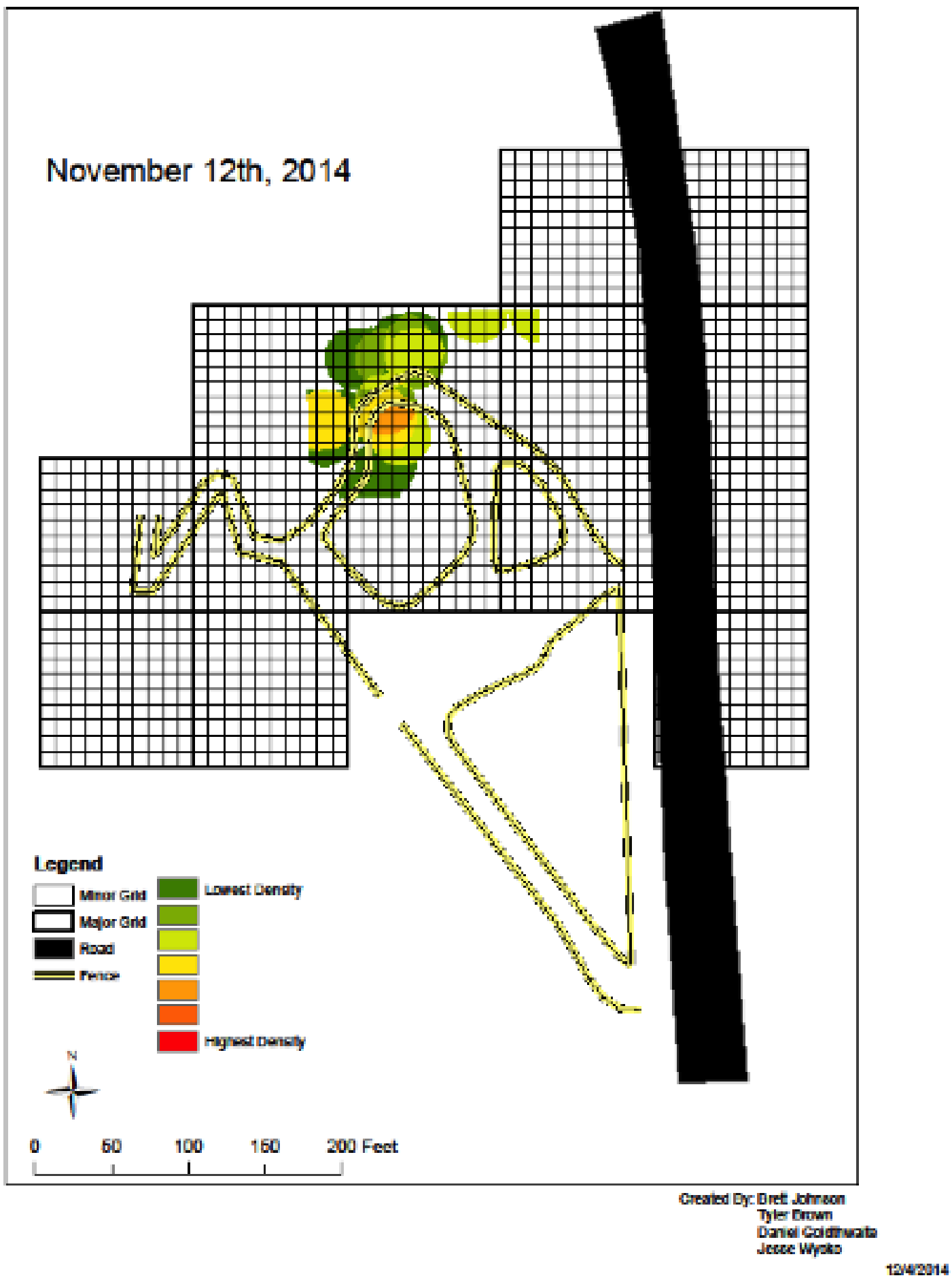




\section{November 2014 - Pismo Monarch Grove Population Density}

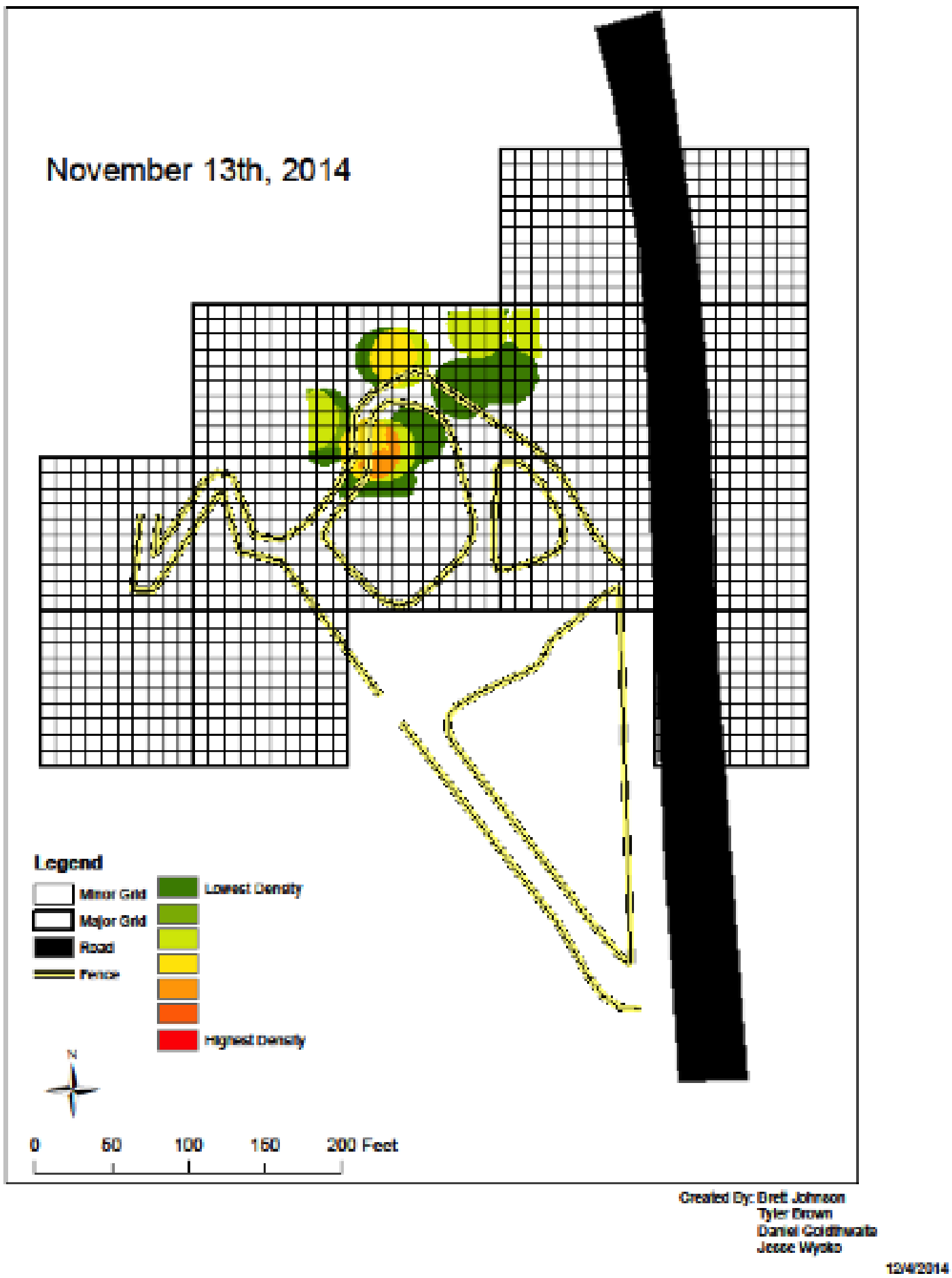




\section{November 2014 - Pismo Monarch Grove Population Density}

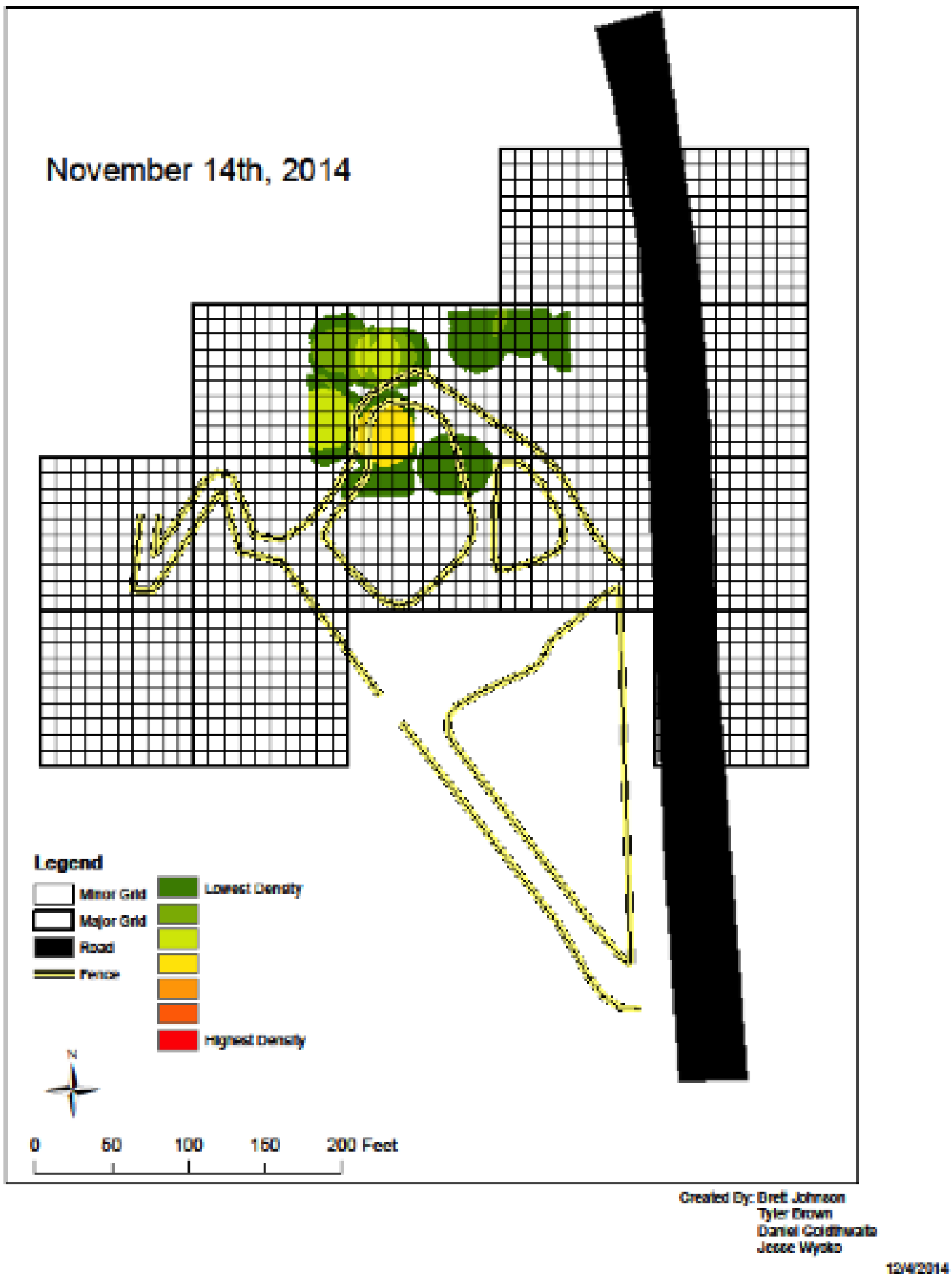




\section{November 2014 - Pismo Monarch Grove Population Density}






\section{November 2014 - Pismo Monarch Grove Population Density}

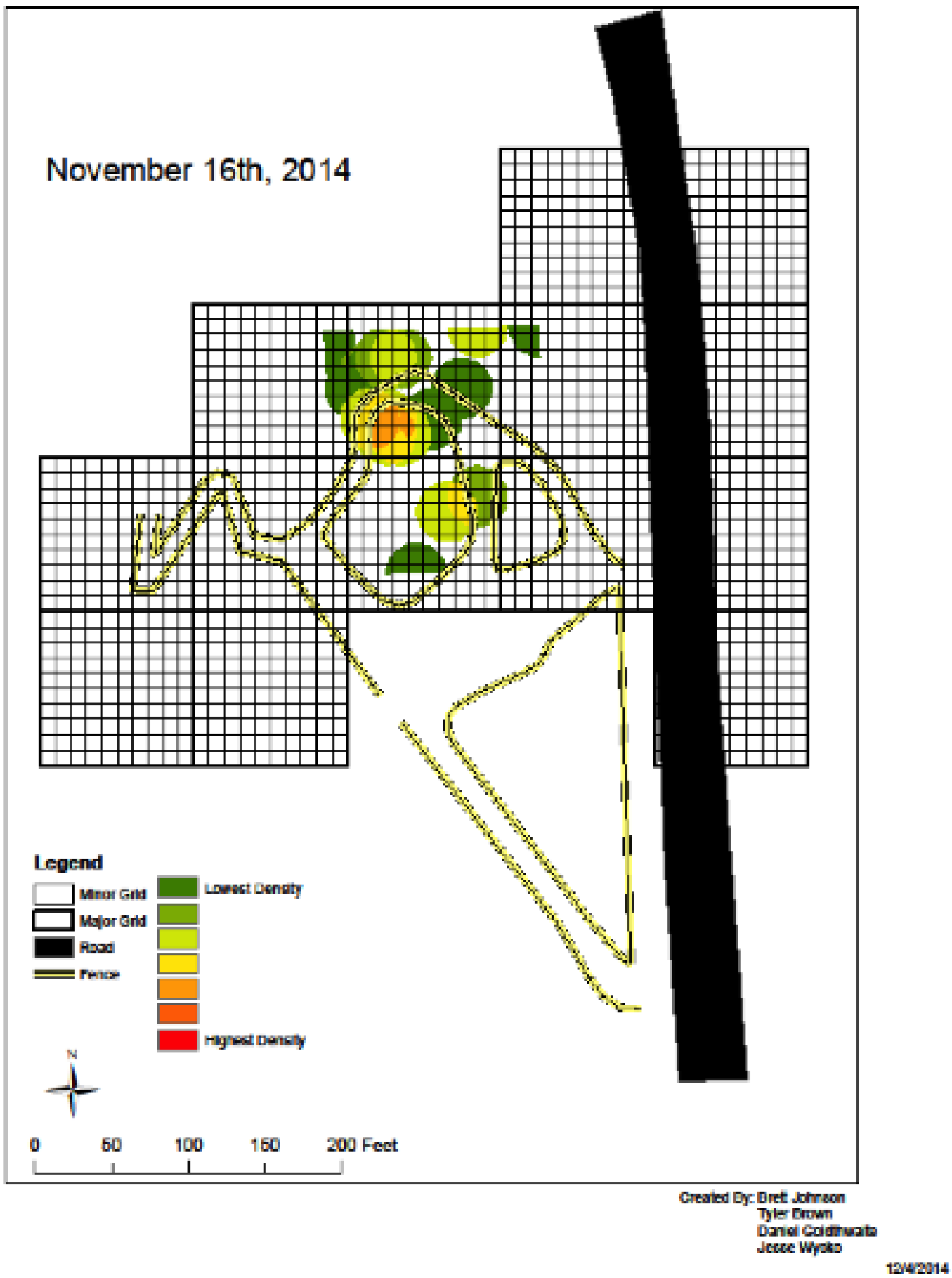


November 2014 - Pismo Monarch Grove Population Density

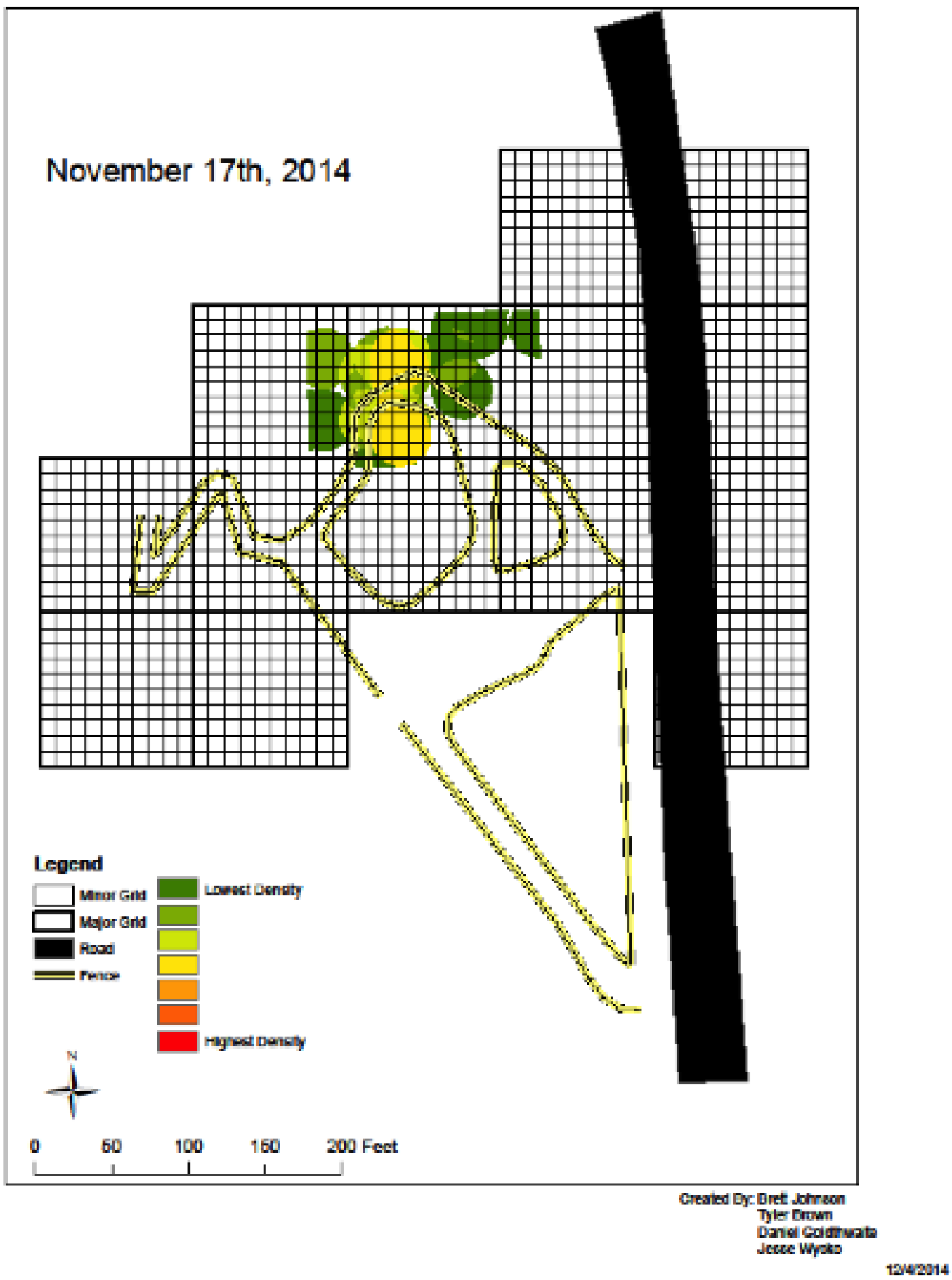




\section{November 2014 - Pismo Monarch Grove Population Density}

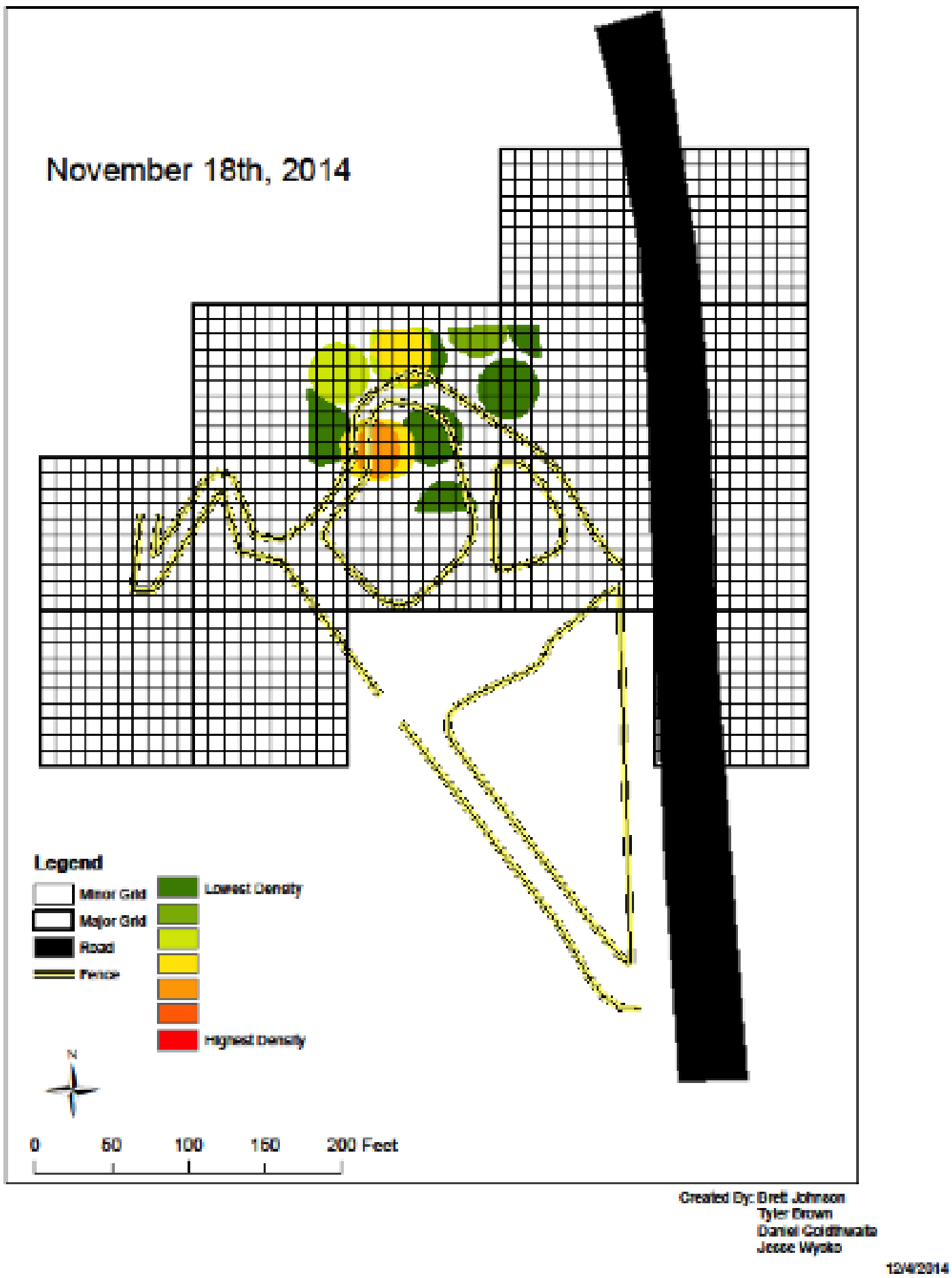


November 2014 - Pismo Monarch Grove Population Density

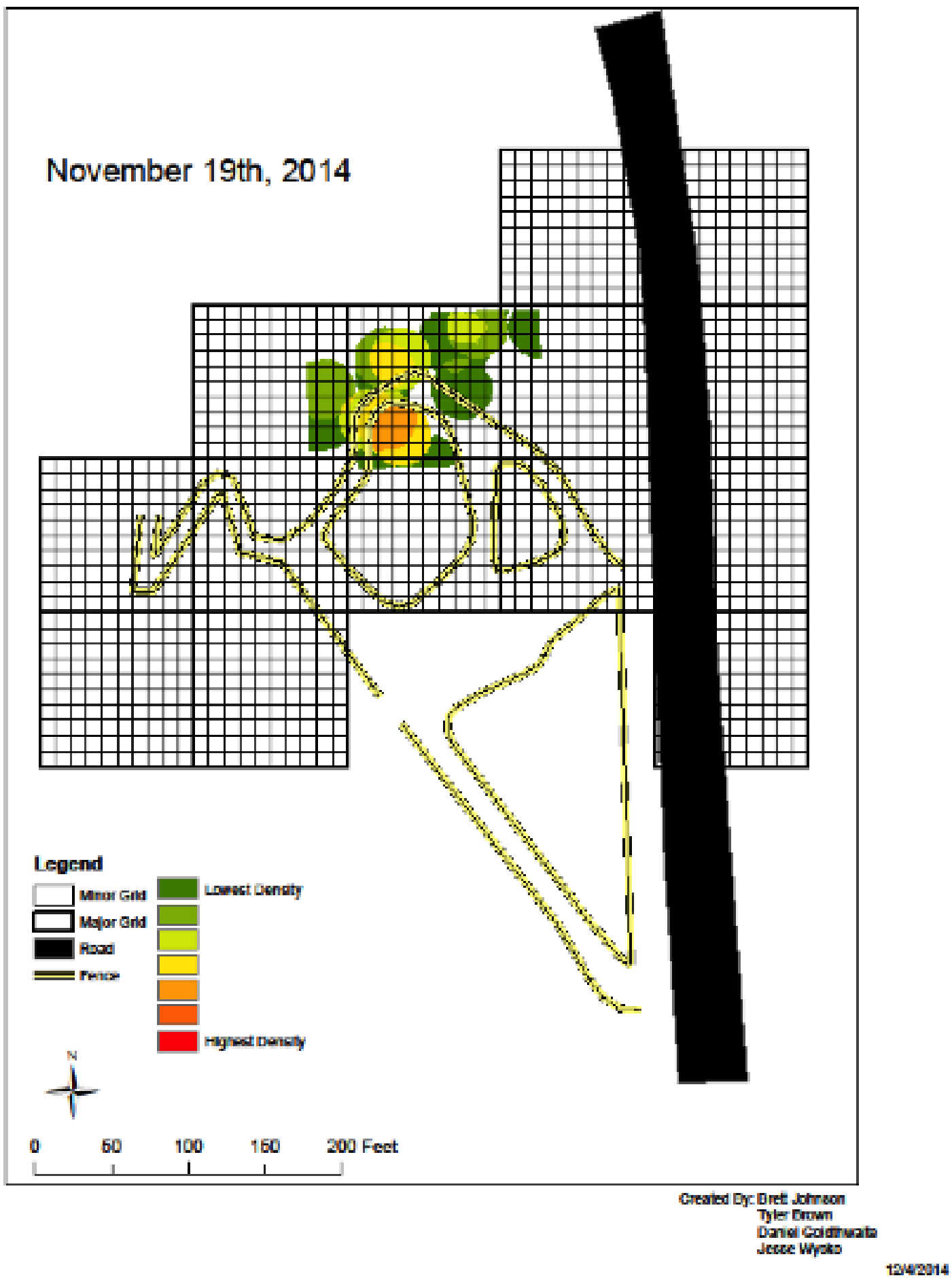




\section{November 2014 - Pismo Monarch Grove Population Density}

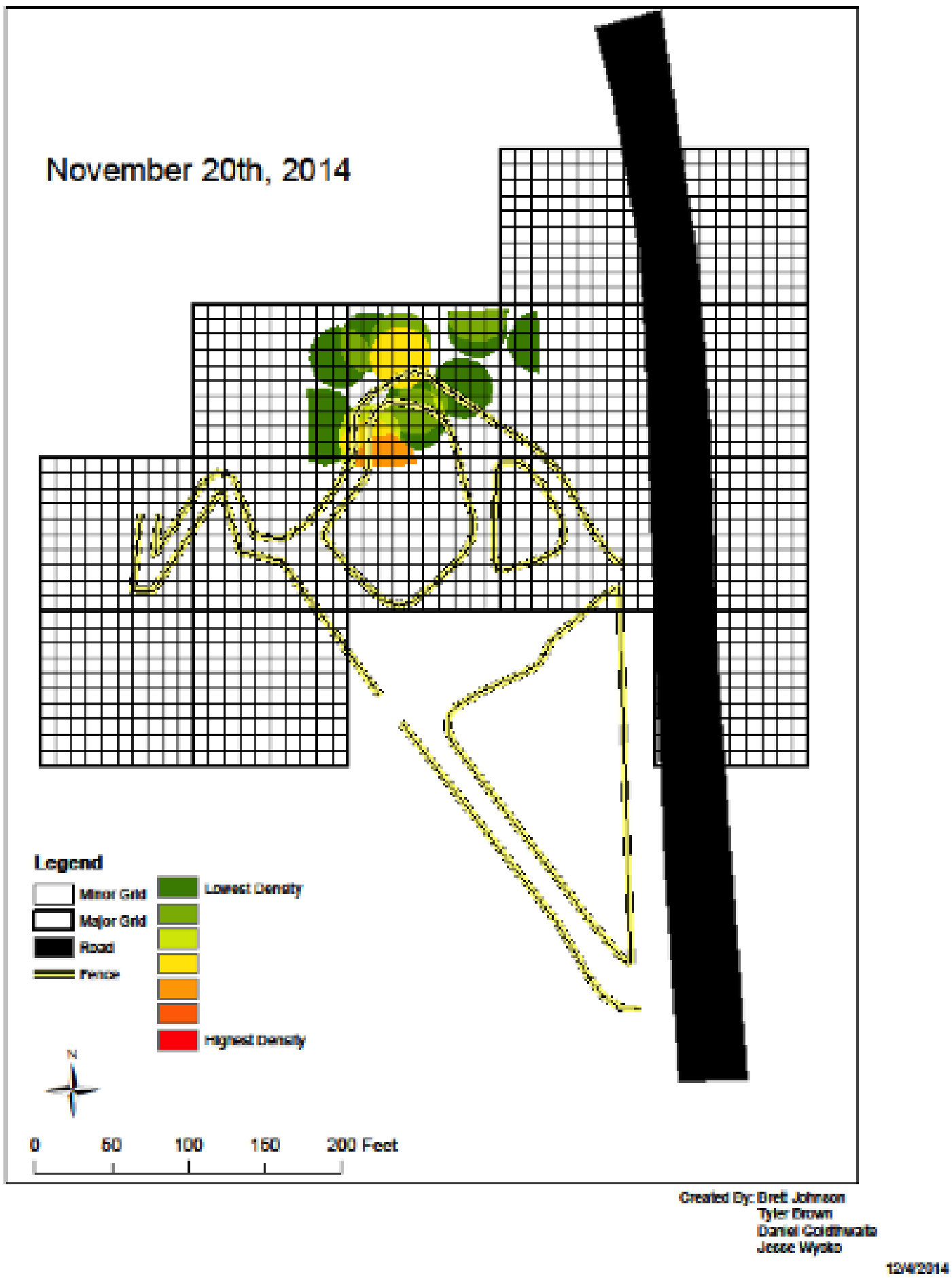




\section{November 2014 - Pismo Monarch Grove Population Density}

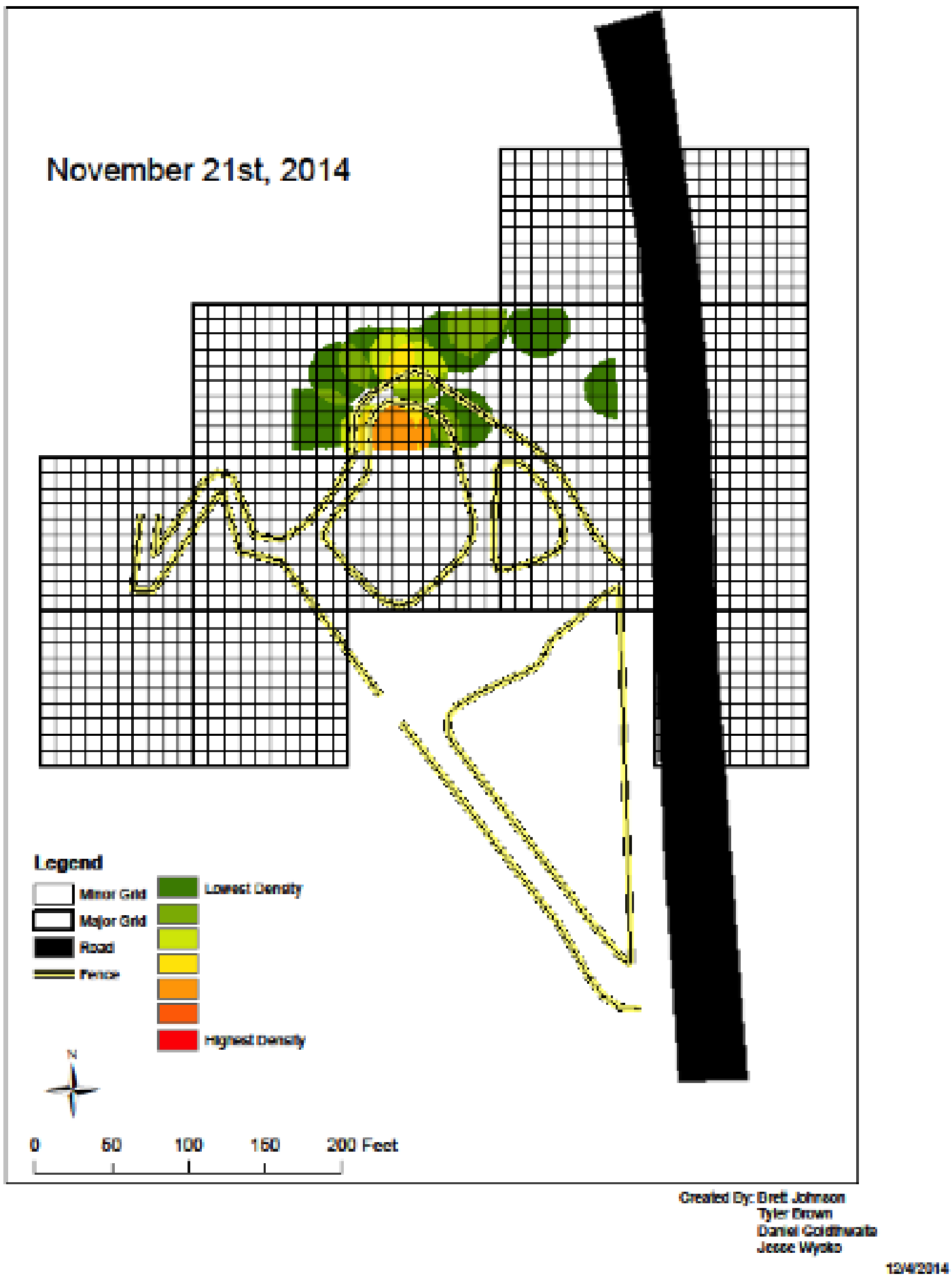


November 2014 - Pismo Monarch Grove Population Density






\section{November 2014 - Pismo Monarch Grove Population Density}

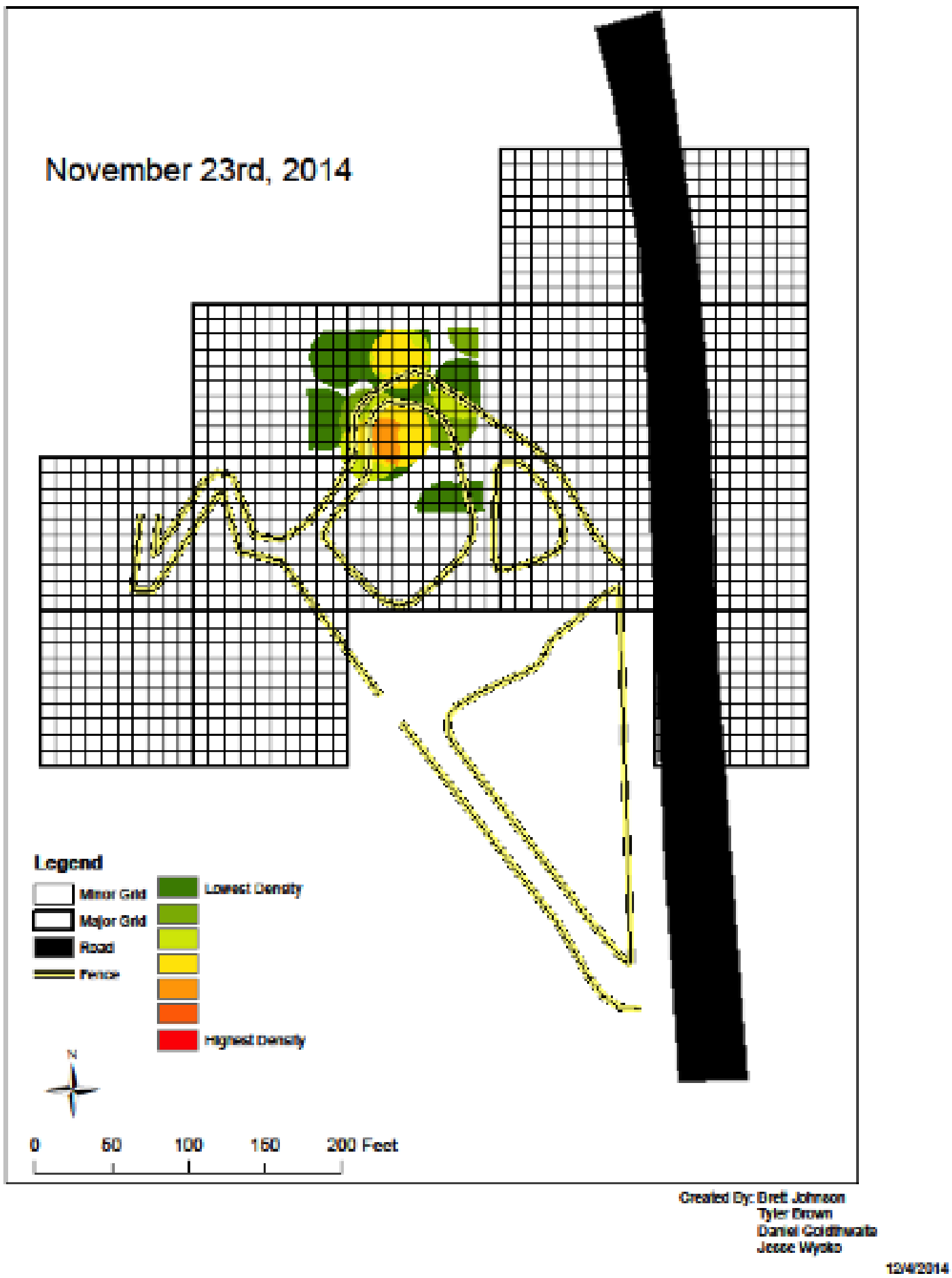




\section{Appendix B}

\section{Over-wintering Monareh Butterfy Project Monarch Count Data Sheet}

DATF IIfifoH STRFAME Prom ÿObserves 1 Ohwervernime(s) Bitt Sohnim

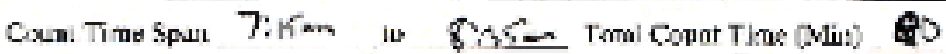

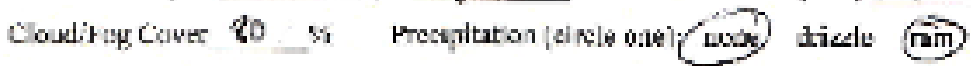
Ienp (c) Wind (iin:4) W'ixal (Teuutirr) 3 Tizd dirostion (yostotit) $\left(-40 \cdot 4 j^{\circ}\right)$

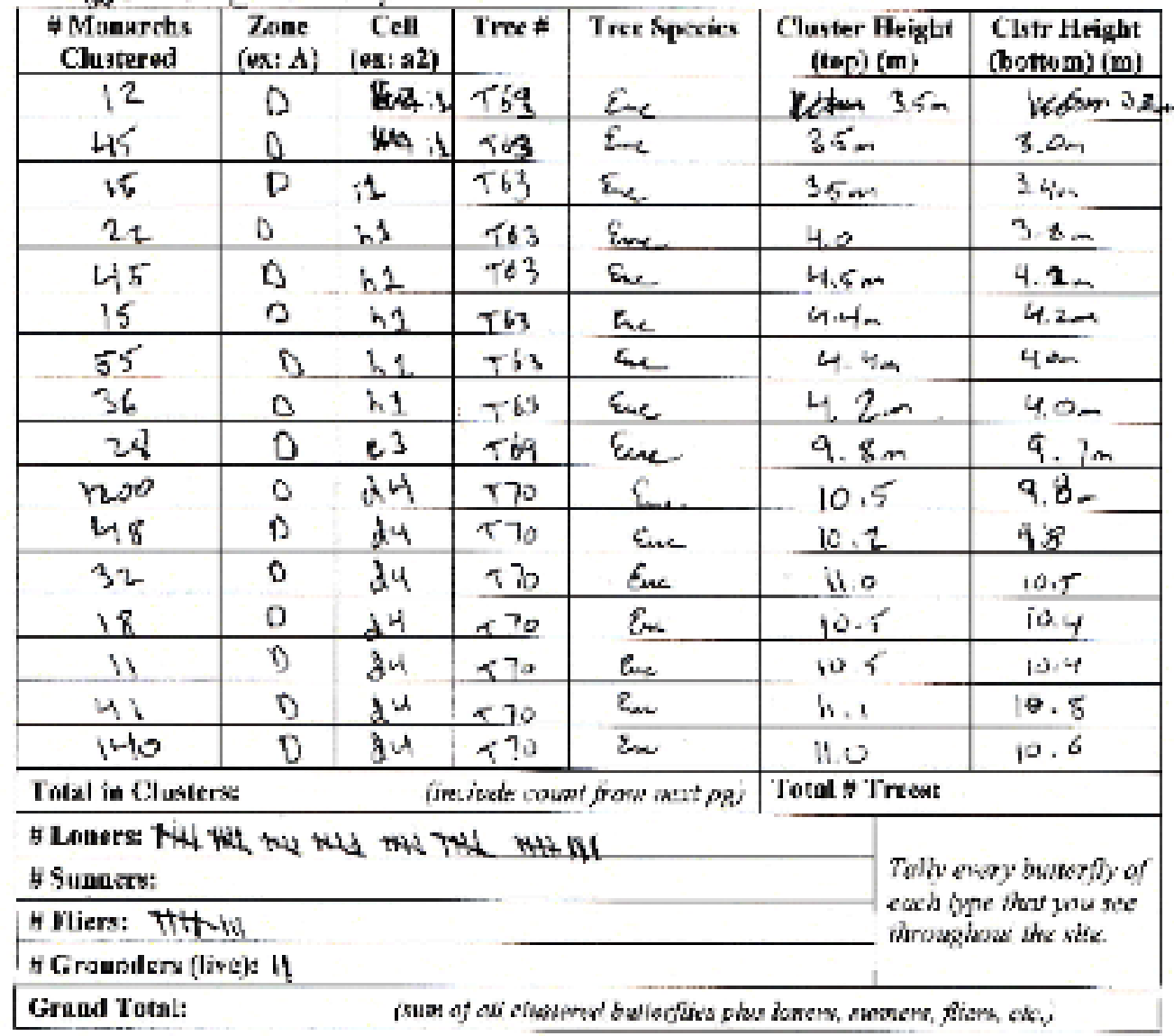

\section{H Mating Munarsho: T}

\# Dead Mlonuruhe:

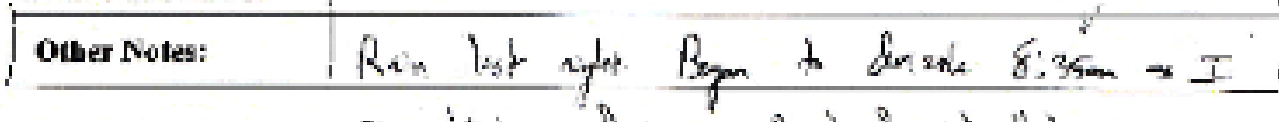

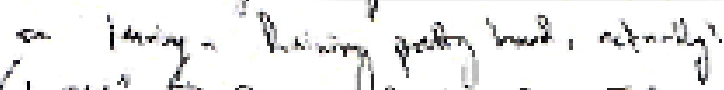

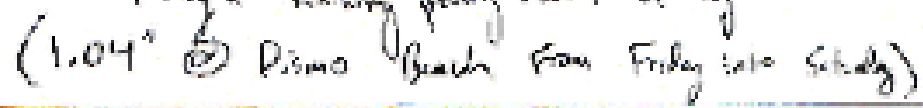




\begin{tabular}{|c|c|c|c|c|c|c|}
\hline $\begin{array}{l}\text { "Menarchs } \\
\text { Clustered }\end{array}$ & $\begin{array}{c}\text { Zone } \\
(\operatorname{man} \wedge)\end{array}$ & $\begin{array}{c}\text { Cell } \\
\left(0 x: x_{2}^{2}\right)\end{array}$ & Tree 4 & Tree spediss & $\begin{array}{c}\text { Cluosur Height } \\
\text { (bsp) (m) }\end{array}$ & \multirow{3}{*}{$\begin{array}{c}\text { Clstr Ieight } \\
\text { (bottom) (1a) } \\
9.8 \\
10,0\end{array}$} \\
\hline 260 & 0 & $\mathrm{CH}^{4}$ & $T T 0$ & Ener & -10.4 & \\
\hline 55 & $D$ & $c^{u_{1}}$ & 170 & Eve & 10,2 & \\
\hline 28 & b & $\mathrm{CH}$ & +70 & Ever & 100 & 9.7 \\
\hline 30 & 0 & 64 & İ7o & $\varepsilon$ & 10.0 & 9.2 \\
\hline 65 & $C$ & $: 8$ & $\langle 3|$ & Gence & 6.8 & 6.8 \\
\hline 14 & $c$ & 18 & 781 & Ever. & 6.5 & 6.7 \\
\hline 40 & $C$ & $i s$ & T है & Ear & 7.4 & 73 \\
\hline 15 & $c$ & 1 & 781 & En & 25 & 7.ب \\
\hline 18 & $c$ & 18 & -981 & Es & 72. & 7.1 \\
\hline 33 & $C$ & 88 & -81 & E.c. & 77 & 20 \\
\hline 30 & $c$ & $i 9$ & $T \$ 1$ & $\varepsilon_{n}$. & 6.8 & 6.7 \\
\hline 110 & $C$ & $\ln 3$ & 794 & En & 8.1 & tax $7^{\circ}$ \\
\hline 80 & $C$ & 68 & $T+T_{1}$ & Ense & 7.8 & -727 \\
\hline 12 & $c$ & $n 8$ & 744 & Ex & 7.8 & 77 \\
\hline 40 & 0 & 60 & $T+0$ & $C_{y p}$ & 8.9 & $7 t$ \\
\hline 35 & 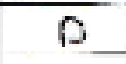 & blo & $r\left(\infty_{0}\right)$ & $\cos p$ & 8.2 & $8 \cdot=$ \\
\hline 12 & $D$ & bid & T100 & & 8.2 & 8.1 \\
\hline 18 & D & bis & $T=0$ & $C_{3}$ & 8.4 & 8.3 \\
\hline 330 & $D$ & $c^{9}$ & 100 & & 12,0 & 1,6 \\
\hline 2.10 & 12 & $\mathrm{ca}$ & $-\tau i a$ & $\cos$ & 12.2 & $1, .7$ \\
\hline 45 & $D$ & 69 & Tov & $\operatorname{lng} \theta$ & 12.2 & 12.0 \\
\hline 95 & 0 & 89 & $\sqrt{100}$ & $c_{a p}$ & 10.3 & 13.1 \\
\hline 22 & $I$ & 2.5 & $T^{n}-12$ & Ens. & 12.2 & 11.9 \\
\hline 16 & $I$ & 95 & THan & Era & 12,0 & 11.9 \\
\hline & & & & & & \\
\hline & & & & & & \\
\hline & & & & & & \\
\hline & & & & & & \\
\hline & & & & & & \\
\hline & & & & & & \\
\hline
\end{tabular}

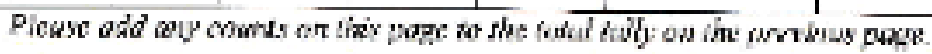




\section{Over-wintering Mounurch Butterfly Project Monareh Count Data Sheet}

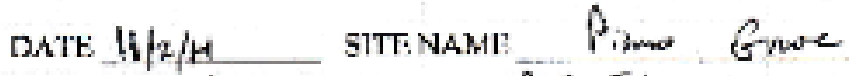

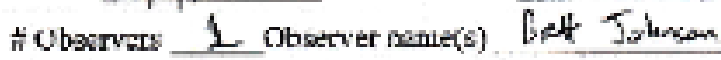



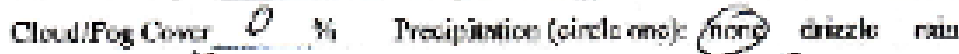

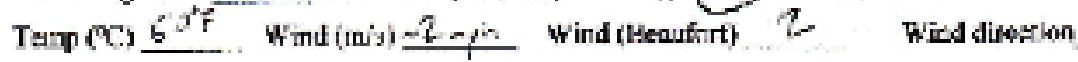

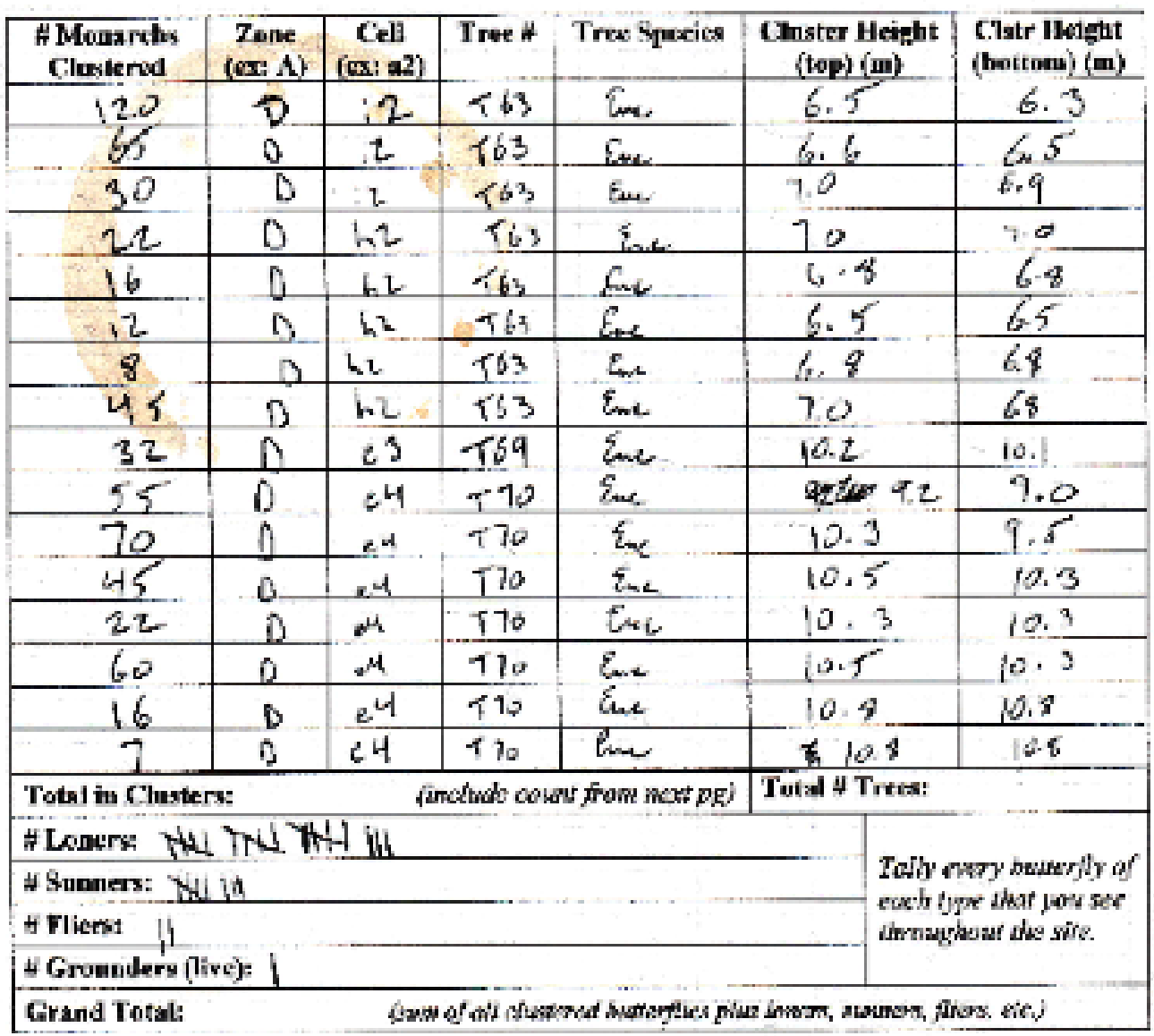

H. Mating Mouaschs:

ff Dead Monarehs:

Other Notes:

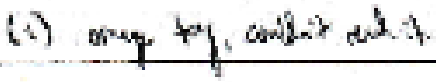




\begin{tabular}{|c|c|c|c|c|c|c|}
\hline $\begin{array}{l}\text { AMowarchs } \\
\text { Clustered }\end{array}$ & $\begin{aligned} \text { Zone } \\
\text { (ux; } A)\end{aligned}$ & $\begin{array}{c}\text { Cell } \\
\left(\mathrm{cx} ; \mathrm{a}_{2}\right) \\
\end{array}$ & Tree \# & Tree spetes & $\begin{array}{c}\text { Chatgr Height } \\
\text { \{top }(\mathrm{m})\end{array}$ & $\begin{array}{l}\text { Clatr Hejeht } \\
(\text { beittum) (m) }\end{array}$ \\
\hline 350 & 0 & 24 & $\{10$ & Ene & 11.0 & 12.8 \\
\hline 60 & $\mathbb{R}$ & $\mu$ & $>-T_{0}$ & 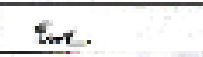 & 1. 2 & 110 \\
\hline 55 & $B$ & 24 & $t+0$ & $\sqrt{\ln 2}$ & 11.5 & $11 .<$ \\
\hline $4-150$ & D & $\mathrm{EH}$ & $T 70$ & $\varepsilon_{2}$ & 12.0 & 160 \\
\hline$i 30$ & 0 & we & $\pi_{0}$ & s. & 11.6 & 11.2 \\
\hline 18 & $D$ & $r_{4}$ & +10 & 2 & 1.6 & 11.6 \\
\hline 28 & 1) & $20 e^{4}$ & 178 & Sace & 10.7 & 16.8 \\
\hline 20 & $D$ & $\cos \mathrm{C}^{4}$ & 70 & Esc & 9.2 & $9 a$ \\
\hline 60 & D & a. $c^{4}$ & $T 70$ & Ear & 9.3 & 9.1 \\
\hline 45 & 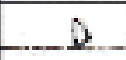 & $c^{4}$ & $r x$ & $E_{\text {esc }}$ & 7.4 & 9,7 \\
\hline 115 & 0 & $\mathrm{c}^{4}$ & 73 & Ene & i. 8 & 9.4 \\
\hline 120 & 0 & 24 & $\tau 70$ & $2 m$ & 10.0 & 4.5 \\
\hline 55 & 0 & 64 & $T_{\text {To }}$ & Ens & 9.8 & 9.7 \\
\hline 10 & $B$ & $\mathrm{CH}$ & $\pi x_{0}$ & Eu & $10 \mathrm{t}$ & 10.1 \\
\hline 30 & D & 64 & $7 ? 0$ & Ser b & 1., 3 & 10.2 \\
\hline 55 & $\hat{D}$ & $e^{+1}$ & -770 & $2-4$ & 4. 4 & 9.6 \\
\hline 22 & D & $c^{4}$ & 770 & $\varepsilon-c$ & 10.0 & 10.0 \\
\hline 325 & 0 & da & $T(0)$ & Cy & 16.0 & 15.0 \\
\hline 250 & $\mathrm{~B}$ & 810 & noso & Gyp & 12.0 & 11.0 \\
\hline 50 & B & . & Tha & aft & 13.5 & 13,0 \\
\hline $3 \alpha$ & D & clo & Tia & c & 13.8 & 13.0 \\
\hline$E 5$ & 0 & Clo & $T i \infty$ & Cu & 12.9 & 13.6 \\
\hline 15 & $\mathrm{O}$ & 010 & $T 100$ & & 13,2 & $1 \leq 0$ \\
\hline 80 & $c$ & .8 & 191 & $\cos$ & 8.1 & 8.0 \\
\hline 25 & C & in & 78 & 6 & g. & 8.0 \\
\hline 20. & $c$ & $i t h$ & $r 84$ & Ene & 7.9 & 78 \\
\hline 50 & $c$ & .8 & $T 81$ & Ears. & 9.2 & 9.0 \\
\hline 站尔 & $c$ & 3 & $T 81$ & in. & 10.5 & 10,4 \\
\hline 130 & $\mathrm{c}$ & 9 & 731 & $\varepsilon$ & 8.0 & 3.2 \\
\hline 60 & c & 71 & 7it. & $E_{n c}$ & 88 & 86 \\
\hline 160 & $c$ & 8 & 78 & are & 11.0 & V.t \\
\hline 175 & $C$ & hq & T.4 & $\mathrm{Gm}$ & 8. 4 & T. \\
\hline 200 & $c$ & ins & $x^{4}$ & $\operatorname{Enc} c$ & 93 & 92 \\
\hline 150 & $M$ & $e 5$ & T1月2 & $E x$ & 100 & 75 \\
\hline \multicolumn{7}{|c|}{ 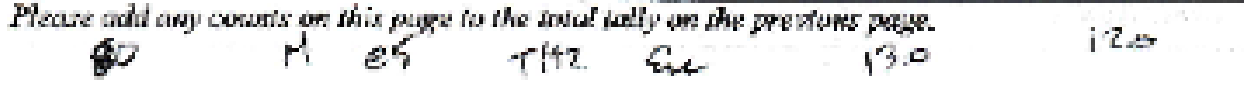 } \\
\hline
\end{tabular}




\section{Over-wintering Monarch Butterfly Project Monurch Count Data Sbeet}

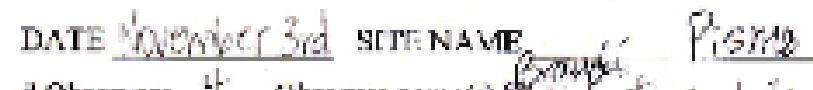

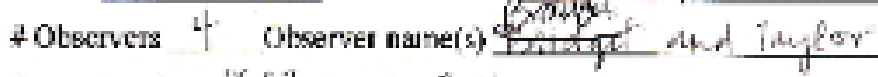

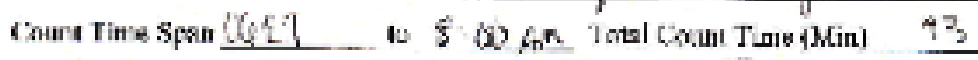

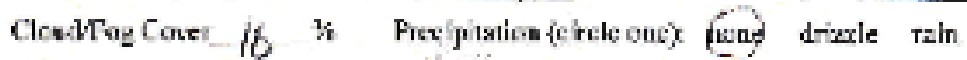

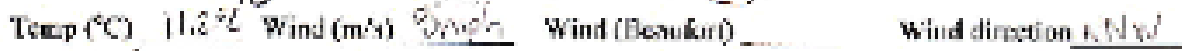

579

\begin{tabular}{|l|l}
\hline \# Meating Monarchy: & \\
\hline Mend Mon arehs: \\
Other Netcs: \\
\hline
\end{tabular}









\section{Over-wintering Monareh Butlerfy Projert Menarch Count Data Sheet}

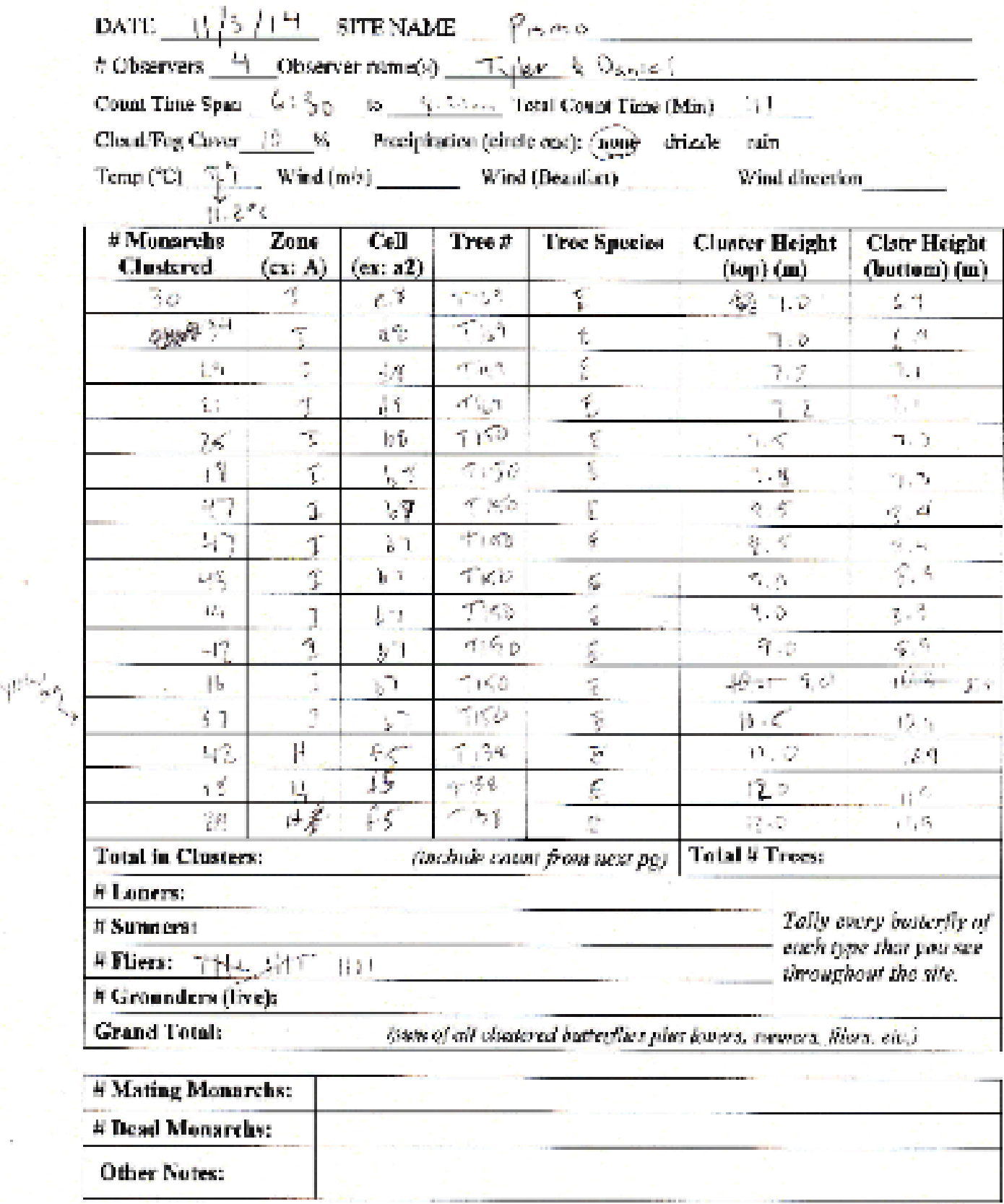




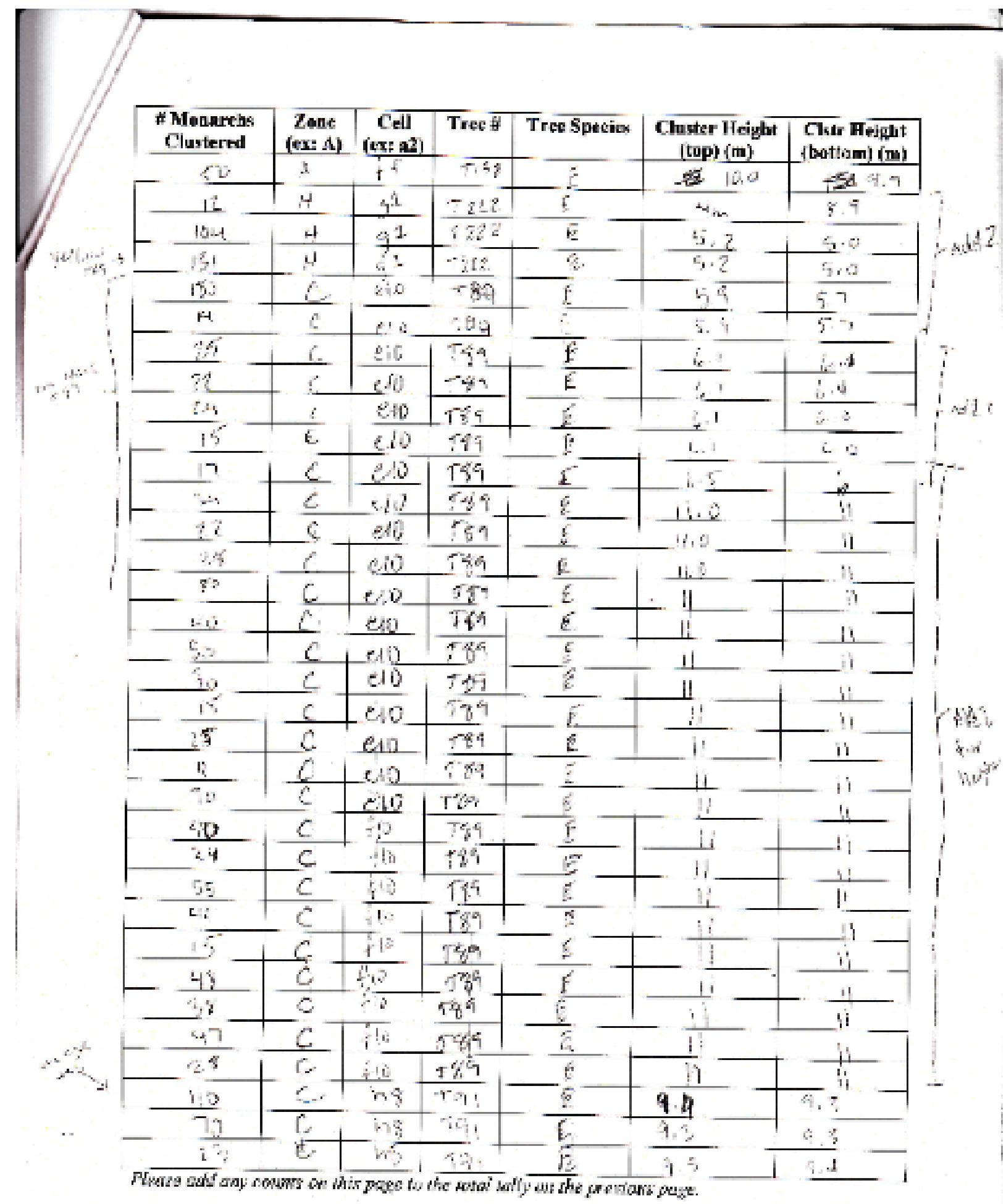




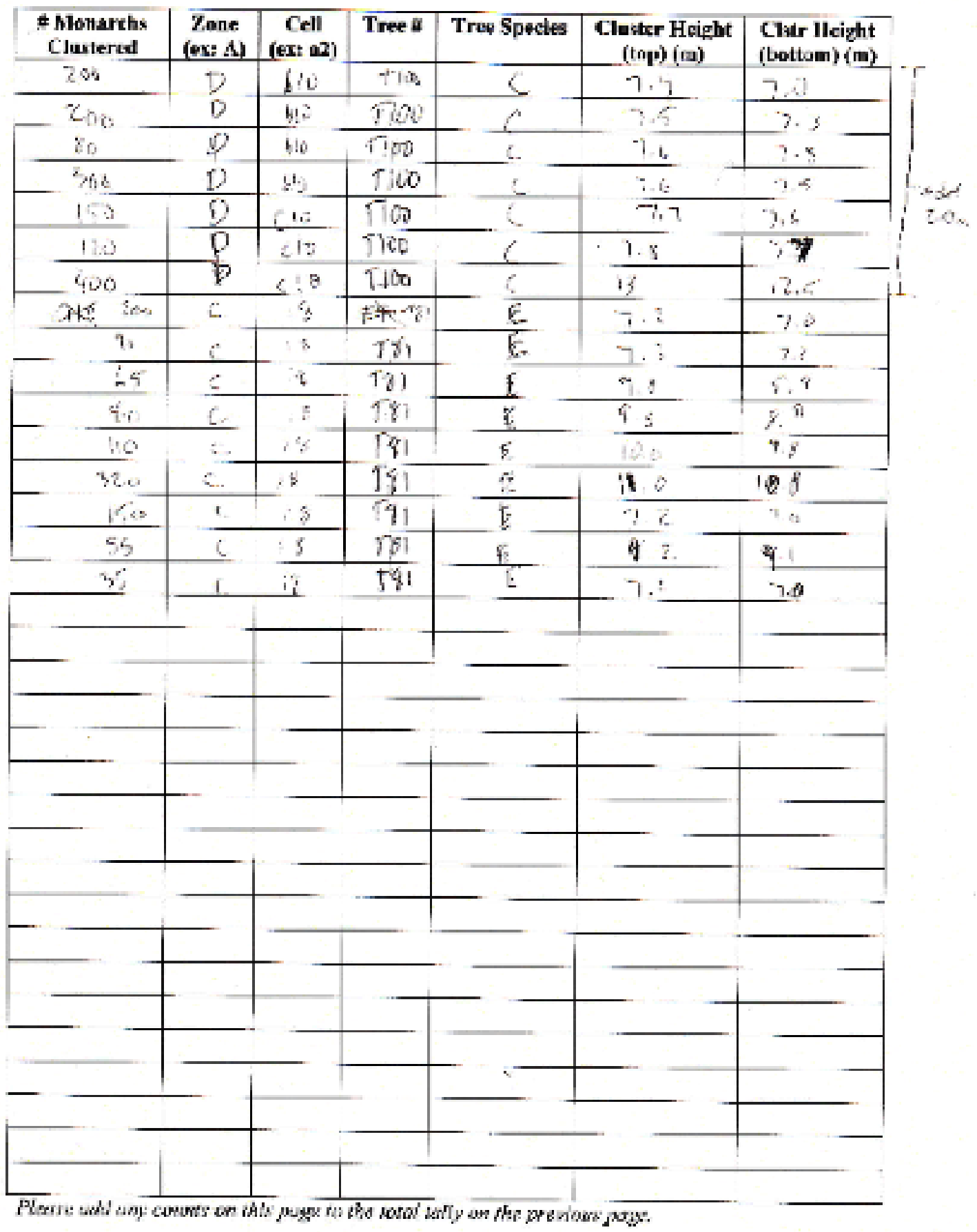




\section{SErited a \\ Over-wintering Monareh Bntterfly Project Monarch Count Data Sheet}

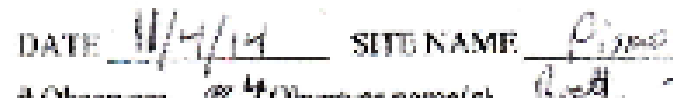

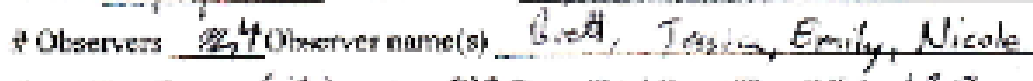

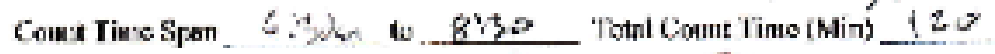

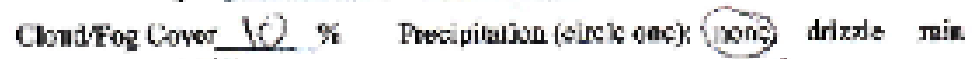

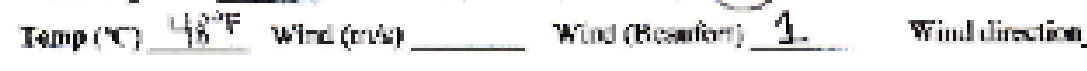

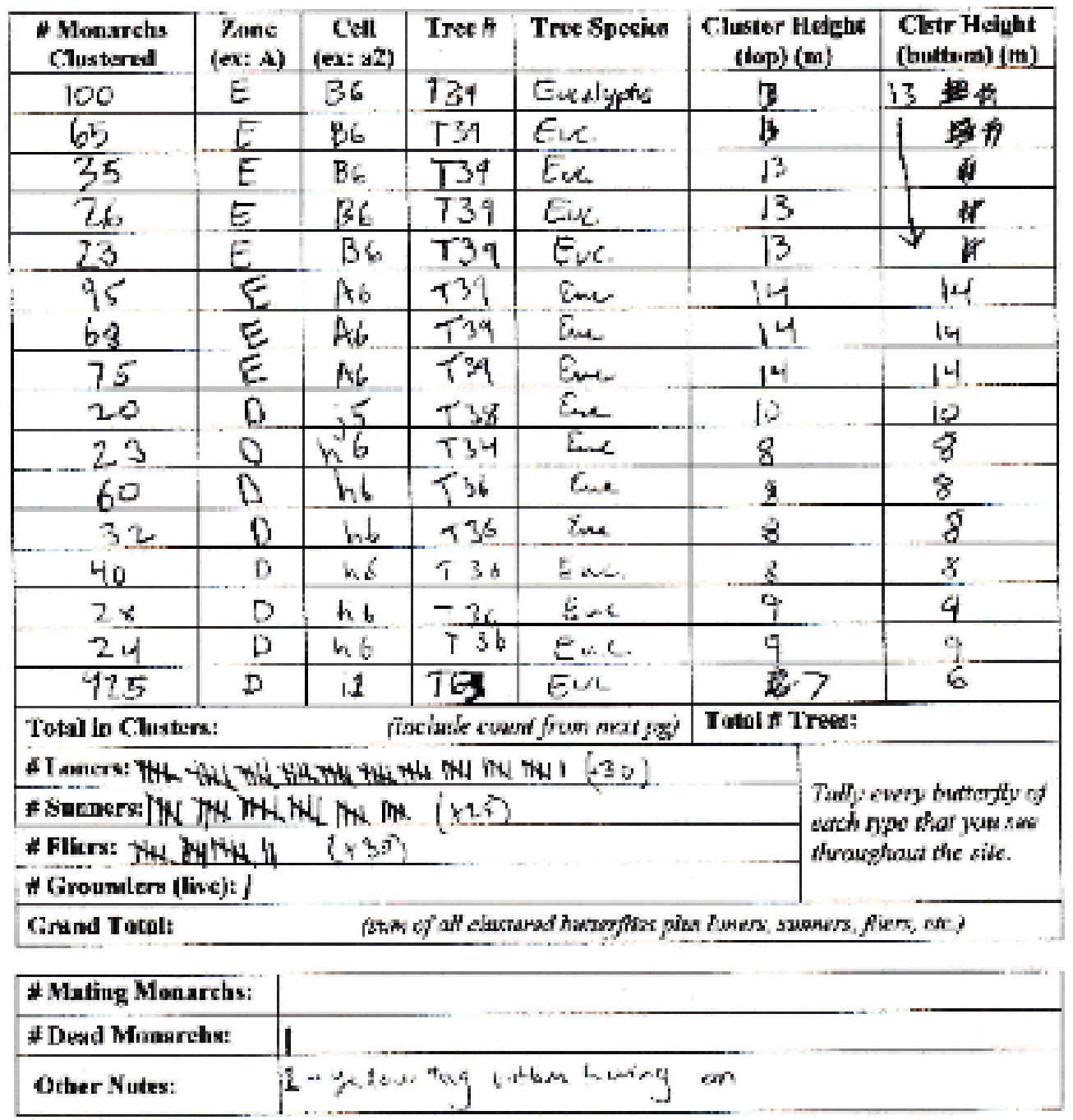




\begin{tabular}{|c|c|c|c|c|c|c|}
\hline $\begin{array}{l}\text { "Monarehs } \\
\text { Clistured }\end{array}$ & $\begin{array}{c}\text { Zone } \\
(m: \text { A) }\end{array}$ & $\begin{array}{c}\text { Cell } \\
\left(\in \times x^{2}\right)\end{array}$ & Tree * & True Species & \begin{tabular}{|c|}
$\begin{array}{c}\text { Clusiter Height } \\
\text { (top) (m) }\end{array}$ \\
\end{tabular} & $\begin{array}{l}\text { Clvir Height } \\
\text { (bottem) (m) }\end{array}$ \\
\hline 300 & $D$ & is & $T=3$ & Eve. & 10 & 9 \\
\hline 425 & $D$ & 1 & TC. 3 & $E \cup C$. & 9 & 8 \\
\hline 175 & $D$ & 1 & $\sqrt{63}$ & Exe. & 11 & $k$ \\
\hline 200 & $D$ & i1 & $T 63$ & Eve. & 8 & $\bar{\delta}$ \\
\hline 130 & $D$ & h1 & 763 & $E_{U C}$. & 9 & 8 \\
\hline 60 & P & $i=1$ & 163 & Eve. & 8 & 8 \\
\hline 850 & $D$ & 69 & Too & $C_{y p r e s s}$ & if & 16 \\
\hline 150 & $D$ & $C 5$ & $T \times \infty$ & $G_{\text {ups }}$ & 17 & 16 \\
\hline 175 & $D$ & C. & T100 & $c_{\text {yer }}$ & 13 & 13 \\
\hline 23 & 0 & cor & $\tau ! \infty$ & Gese & 13 & 13 \\
\hline 575 & $D$ & 49 & 7160 & $\Delta_{4 y}^{n}$ & 12 & 12 \\
\hline 150 & $I A$ & $d t$ & 5100 & Ge & 12 & 12 \\
\hline 425 & $D$ & $d^{4}$ & 170 & $e x$ & 4 & $y$ \\
\hline 900 & 9 & $d 4$ & 170 & $e x$ & 12. & It. \\
\hline 300 & $D$ & A & 720 & eut & $1 i$ & in \\
\hline 40 & D & bs & $T 6^{9}$ & eve & $2 i$ & 21 \\
\hline 30 & D & 15 & $\pi \%$ & cos & 7.1 & 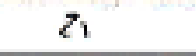 \\
\hline 100 & $D$ & 65 & $T 69$ & eve & 20 & $z=y$ \\
\hline 90 & 2 & 65 & 170 & eut & $n$ & 011 \\
\hline$a_{0}$ & D) & 55 & $T 70$ & Pux & $i$ & II \\
\hline $2 k$ & $\hat{y}$ & 65 & 770 & eyc & 10 & 10 \\
\hline 70 & $\nabla$ & 65 & 770 & pók & is & 10 \\
\hline 55 & 1). & $\Delta T$ & $r>0$ & Puc & yi & if \\
\hline 130 & 6 & is & $T \& 1$. & $E, z_{2}$ & $g$ & $x$ \\
\hline 53 & $c$ & ;9 & $T 81$ & Eus. & 8 & $\&$ \\
\hline 80 & $c$ & 18 & $T \& 1$ & Euc & 9 & 9 \\
\hline 80 & $c$ & 느 & +44 & Evc & 12 & $1-2$ \\
\hline 60 & $c$ & $\mathrm{Ha}^{\circ}$ & T44 & En & 3 & 13 \\
\hline 70 & c & Hqㅗ & T94 & Eus & 13 & 13 \\
\hline 130 & it & $\mathrm{Gr}^{2}$ & $-2^{2} 2$ & Eus & 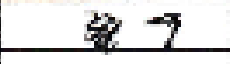 & 7 \\
\hline 20 & 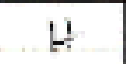 & $G t$ & $T \pm 2 z$ & Fic & 7 & 7 \\
\hline 70 & it & 61 & $F$ Ken & Enes & 10 & 10 \\
\hline 7.90 & 1 & Gl & Filler & Eree & 32 & 12 \\
\hline 80 & Ht & 85 & r, 31 & Ene & 12 & 12 \\
\hline \multicolumn{7}{|c|}{ 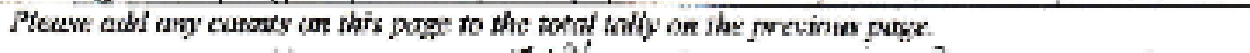 } \\
\hline $\begin{array}{l}50 \\
47\end{array}$ & it & $\begin{array}{l}\text { G9 } \\
\text { C10 }\end{array}$ & $\begin{array}{l}T 186 \\
T 1 \& 4\end{array}$ & $\frac{E_{u}}{\xi+c .}$ & 8 & $\begin{array}{l}5 \\
7\end{array}$ \\
\hline
\end{tabular}


- Entend

\section{Over-wintering Monarch Butterfly Project Monarch Count Data Sheet}

DATE $11 / 5 / M$ SITE NAME Pisno Rerch

y Observers__Obsenver name(s) Tyler, Brand: Tryle

Count Time Spen $6: 15$ to $7: 50$ Total Count Time (Min) 95

CloudFog Cover__ \% Precipitation (circle one) $\%$ noms drizzle min

Tenp $\left({ }^{\circ} \mathrm{C}\right)$ 16.1 Wind (mis) +3 Wind (Beasufor) ___ Wind dirextion_

\begin{tabular}{|c|c|c|c|c|c|c|c|c|}
\hline & $\begin{array}{l}\text { Monarehs } \\
\text { Clustered }\end{array}$ & $\begin{array}{c}\text { Zone } \\
(\mathbf{e x :} A)\end{array}$ & $\begin{array}{c}\text { Cell } \\
\left.\text { (ex: } \mathrm{a}^{2}\right)\end{array}$ & Tree "I & Tree Species & \multicolumn{2}{|c|}{$\begin{array}{l}\text { Cluster Height } \\
\text { (top) (m) }\end{array}$} & $\begin{array}{l}\text { Csstr Height } \\
\text { (bettom) (m) }\end{array}$ \\
\hline$n_{3}$ & 307 & $\xi$ & 27 & 739 & $E$ & \multicolumn{2}{|c|}{13} & $12 \mathrm{~m}$ \\
\hline & 40 & ti & $a b$ & 739 & 2 & \multicolumn{2}{|c|}{12} & $12 \mathrm{~m}$ \\
\hline & 85 & $D$ & $h 6$ & 134 & $\varepsilon$ & \multicolumn{2}{|c|}{1} & $10 \mathrm{~m}$ \\
\hline & 140 & $D$ & h6 & $T 34$ & $E$ & \multicolumn{2}{|c|}{11} & $11 \mathrm{~m}$ \\
\hline & 170 & $D$ & i1 & $T 63$ & $E$ & \multicolumn{2}{|c|}{12} & $12 \mathrm{~m}$ \\
\hline & 200 & $D$ & $i 1$ & $T_{b 3}$ & $E$ & \multicolumn{2}{|l|}{$g$} & $8 m$ \\
\hline & 25 & $\nabla$ & $h^{1}$ & 963 & $E$ & \multicolumn{2}{|c|}{8} & $\delta \mu$ \\
\hline & 180 & $D$ & h1 & 763 & $E$ & \multicolumn{2}{|c|}{15} & $15 \mathrm{M}$ \\
\hline & $9 b$ & D & $h 1$ & 163 & $E$ & \multicolumn{2}{|c|}{13} & $13 \mathrm{~m}$ \\
\hline & 200 & D & $h 1$ & $\pi 3$ & $E$ & \multicolumn{2}{|l|}{11} & $11 \mathrm{~m}$ \\
\hline & 270 & $D$ & d 3 & 170 & $E$ & \multicolumn{2}{|c|}{10} & $10 \mathrm{~m}$ \\
\hline & 65 & D & $d 3$ & $T 70$ & E & \multicolumn{2}{|c|}{14} & $14 \mathrm{~m}$ \\
\hline & 75 & $D$ & $d 4$ & 770 & $\bar{E}$ & \multicolumn{2}{|l|}{ n } & $11 \mathrm{~m}$ \\
\hline & 375 & D & 15 & 570 & $\frac{1}{2}$ & \multicolumn{2}{|l|}{11} & $10 \mathrm{~m}$ \\
\hline & 380 & $D$ & 65 & 170 & $E$ & \multicolumn{2}{|l|}{11} & $10 \mathrm{~m}$ \\
\hline & 215 & D & 65 & 770 & $E$ & \multicolumn{2}{|c|}{10} & $10 \mathrm{~m}$ \\
\hline & \multicolumn{5}{|c|}{ Incinde count from noxt $\mathrm{pg}$ ) } & \multicolumn{3}{|c|}{ Total \# Trees: } \\
\hline & \multicolumn{5}{|c|}{ 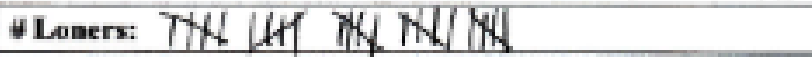 } & \multirow{4}{*}{$\begin{array}{l}\text { Tally ewery butterfity of } \\
\text { each type that jou see } \\
\text { throughout the site. }\end{array}$} & \multirow{4}{*}{\multicolumn{2}{|c|}{$\begin{array}{l}\text { Tally ewery butterfity of } \\
\text { earh igpe that you see } \\
\text { throughout the site. }\end{array}$}} \\
\hline & \multicolumn{5}{|l|}{ y Sunners: } & & & \\
\hline & \#Fliers: & IXX & IUX & & & & & \\
\hline & \multicolumn{5}{|c|}{ \# Graanders (live): $\not b$} & & & \\
\hline & \multicolumn{5}{|c|}{ all clustered bmikerfities oll } & s beers, sw & mers, $f$ : & ra. edc.) \\
\hline
\end{tabular}

\begin{tabular}{|l|l|}
\hline \# Mating Monarchs: & $\varnothing$ \\
\hline W Dead Monarehs: & $\varnothing$ \\
\hline Other Notes: & \\
\hline
\end{tabular}









\section{$\not 1$ Entred}

\section{Over-wintering Monarch Butterfly Project Monarch Count Data Sheet}

DATE $\Perp / 6 / 14$ SITENAME Pismo

4 Observers 3 Observer name(s) Brett, Emily, Nicole

Count Time Spen $6: 20$ w $8: 00$ Total Count Time (Min) 100

Cloud/Fog Coner $10 \%$ Precipitation (circle one): (rongl drizle rain

Temp ('C) 3.5 Wind $(\mathrm{m} / \mathrm{s}) \mathrm{Q} . \mathrm{S}^{\mathrm{m} / \mathrm{s}}$ Wind (Benafort) ___ Wind direction NE

\begin{tabular}{|c|c|c|c|c|c|c|c|}
\hline $\begin{array}{c}\text { A Monarehs } \\
\text { Clustered }\end{array}$ & \begin{tabular}{|c|} 
Zone \\
(ex: A)
\end{tabular} & \begin{tabular}{|c|} 
Cell \\
(ex: $\left.a_{2}\right)$
\end{tabular} & Tree A & Tree Species & \multicolumn{2}{|c|}{$\begin{array}{l}\text { Cluster Height } \\
\text { (top) (m) }\end{array}$} & $\begin{array}{l}\text { Clstr Height } \\
\text { (bottom) (m) }\end{array}$ \\
\hline $.55,55(55)$ & $E$ & 66 & $T 40$ & Ex. & \multicolumn{2}{|c|}{$\frac{1}{9}$} & 9 \\
\hline $22,25(24)$ & $E$ & 66 & 740 & Ene & \multicolumn{2}{|c|}{10} & $10^{\circ}$ \\
\hline $40,60(15)$ & $E$ & $b 6$ & Tho & $\varepsilon_{x_{x}}$ & \multicolumn{2}{|c|}{10} & 10 \\
\hline $10,13(12)$ & E & $a b$ & T40 & Ene & \multicolumn{2}{|c|}{6} & 6 \\
\hline $24,30(27)$ & E & 96 & T40 & Ene & \multicolumn{2}{|c|}{6} & 6 \\
\hline $20,22(21)$ & E & 96 & 140 & Ene & \multicolumn{2}{|c|}{6} & 6 \\
\hline $40,50(45)$ & $E$ & 96 & 140 & Ene & II & & 11 \\
\hline $60,70(65)$ & $E$ & .05 & $T 38$ & Eve & \multicolumn{2}{|c|}{$\frac{11}{8}$} & 8 \\
\hline 75,80771$)$ & 0 & he & $T 34$ & Eve & \multicolumn{2}{|c|}{9} & 9 \\
\hline $120,170(\mathrm{~m} / \mathrm{s})$ & $\Delta$ & h6 & T3- & Esc. & \multicolumn{2}{|l|}{10} & 10 \\
\hline $12,22(22)^{\prime}$ & $D$ & h6 & 134 & Ene & \multicolumn{2}{|l|}{10} & 10 \\
\hline $24,25(25)$ & D & he & 134 & Ene & \multicolumn{2}{|l|}{10} & 10 \\
\hline$(0,105,(10)$ & D & $h_{0}$ & $T 34$ & Euc & \multicolumn{2}{|l|}{10} & 10 \\
\hline $50,55 \quad(53)$ & D & 46 & T34 & Ew & \multicolumn{2}{|l|}{10} & 10 \\
\hline $80,80 \quad(30)$ & D & $h 6$ & 734 & Ek & \multicolumn{2}{|l|}{$\pi$} & II \\
\hline $1 / 19 \quad(10)$ & D & 46 & 734 & En & \multicolumn{2}{|l|}{ 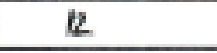 } & L \\
\hline \multicolumn{5}{|c|}{ nclude conum from mex } & \multicolumn{3}{|c|}{ Total Hi Trees: } \\
\hline \multicolumn{5}{|c|}{ 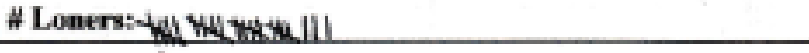 } & & \\
\hline \# Sunnerss Thaty & & & & & \multirow{3}{*}{$\begin{array}{l}\text { Tally every buaterlly of } \\
\text { each type that you see } \\
\text { throughous the stre. }\end{array}$} & & \\
\hline \multicolumn{5}{|c|}{ 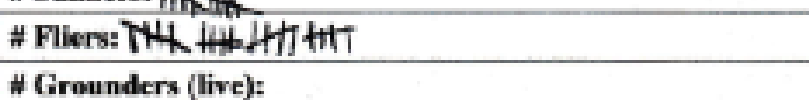 } & & & \\
\hline \multicolumn{5}{|c|}{ \# Grounders (live): } & & & \\
\hline Grand Total: & & & $f a l l c h$ & butherple & at loner: & ner. & ers, act) \\
\hline
\end{tabular}

\begin{tabular}{|c|c|}
\hline \# Mating Monarchs: & 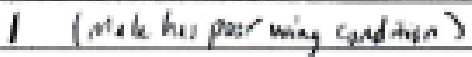 \\
\hline \# Dead Monarehs: & \\
\hline Other Notes: & \\
\hline
\end{tabular}




\begin{tabular}{|c|c|c|c|c|c|c|}
\hline $\begin{array}{l}\text { Monarchs } \\
\text { Clustered }\end{array}$ & $\begin{array}{c}\text { Zene } \\
(\mathrm{ex}: \mathrm{A})\end{array}$ & $\begin{array}{c}\text { Cell } \\
(\mathrm{ex}: 22)\end{array}$ & Tree \# & Tree Species & $\begin{array}{l}\text { Clester Height } \\
\text { (top) (m) }\end{array}$ & $\begin{array}{l}\text { Clstr Height } \\
\text { (bottom) (m) }\end{array}$ \\
\hline $5065 \quad \operatorname{kno}(5 v)$ & $D$ & hb & T34 & EvL & 11 & II \\
\hline 45,46 (15) & $D$ & $h_{6}$ & $T 34$ & Eve & 11 & II \\
\hline $100,100 \quad(100)$ & $D$ & $h 6$ & 734 & Eve & 12 & 12 \\
\hline $675,600(635)$ & D & i1 & $T 63$ & Eve & 47 & 6 \\
\hline $50,60(55)$ & 0 & if & $\mathrm{T} 63$ & Ene & 6 & 6 \\
\hline $80,110(95)$ & 0 & $i 1$ & $T 63$ & $\varepsilon$ & 6 & 6 \\
\hline $70,60(75)$ & D & i1 & $T 63$ & euc & 7 & 46 \\
\hline $65,65(65)$ & 0 & $h 1$ & +63 & Ene & 6 & $\$ 6$ \\
\hline $260,300(20)$ & $D$ & i1 & $T 63$ & Ene & 11 & 10 \\
\hline $1000,1100(1099)$ & D & i1 & $T 63$ & Ene & 13 & 10 \\
\hline $400,450(425)$ & $D$ & i2 & $T 63$ & Ec & II & 9 \\
\hline 92,80 (86) & D & 24 & $T 70$ & Ev & 86 & 5 \\
\hline (15) & $H$ & h1 & fle & $\varepsilon_{2}$ & 6 & 6 \\
\hline $140,180(160)$ & D & $d y$ & T70 & Eve & 67 & 65 \\
\hline$(250)$ & $C$ & 48 & Tatz $z x$ & Ene & 11 & 10 \\
\hline$(125)$ & C & 68 & Tates & Ene & 12 & 10 \\
\hline$(275)$ & C & 48 & Thun & Ene & 13 & 11 \\
\hline $400450(425)$ & $D$ & $d y$ & TYO & Euc & 10 & 9 \\
\hline (35) & C & $i 9$ & $T 81$ & Eve & 7 & 7 \\
\hline$(300)$ & C & $; 8$ & $T \$ 1$ & $\varepsilon_{c}$ & 12 & II \\
\hline $420,550(485)$ & 0 & 23 & $T 70$ & Eme & 12 & 10 \\
\hline (15) & $c$ & is & $T 81$ & Gne & 8 & 8 \\
\hline $525600(575)$ & D & $d^{3}$ & 770 & Euc & II & 10 \\
\hline $200,210(240)$ & D & $d^{4}$ & 169 & Evc & 19 & 19 \\
\hline $0,90 \quad(80)$ & $D$ & $d^{h}$ & 769 & Euc & 19 & 19 \\
\hline $600,700(650)$ & 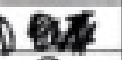 & c9 & 7700 & $C_{p p}$ & 10 & 1 \\
\hline $400,450 \quad(175)$ & D & Cd & 7100 & $\mathrm{Cyp}_{\mathrm{f}}$ & 11 & 10 \\
\hline $270,350 \quad(300)$ & $D$ & (9) & 1100 & up & 11 & II \\
\hline $220,250(233)$ & D & C9 & noo & Cyp & 13 & 13 \\
\hline $325331 \times 0(333)$ & D & C9 & T100 & cyp & 1617 & 16 \\
\hline $20,300(200)$ & D & $C 3$ & Tาo & Enc & 12 & 14 \\
\hline & & & & & & \\
\hline & & & & & & \\
\hline
\end{tabular}

Please add any coumts on this page to the fotal tally on the previons page. 


$$
\text { If extred }
$$

\section{Over-wintering Monarch Butterfly Project Monarch Count Data Sheet}

DATE $11 / 7 / 14$ SITENAME $0.5 \mathrm{~mm}$

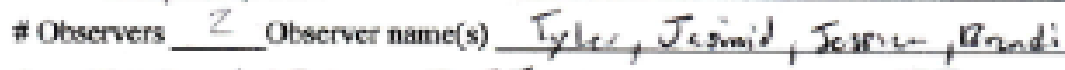

Count Time Span $6: 20$ * 7:35 Toual Count Time (Min) 75

Cloud/Fog Cover 50\% \% Precipitation (sircle one): nons drizzle rain

Temp ("C) 14.2 Wind (m/s) 0.1 Wind (Beaufort) ___ Wind direction

\begin{tabular}{|c|c|c|c|c|c|c|}
\hline $\begin{array}{l}\text { Meanarchs } \\
\text { Clustered }\end{array}$ & $\begin{array}{c}\text { Zome } \\
\text { (ex: A) }\end{array}$ & $\begin{array}{c}\text { Cell } \\
\text { (ex: a2) }\end{array}$ & Tree A & Tree Speeies & $\begin{array}{l}\text { Cluster Height } \\
\text { (top) (m) }\end{array}$ & $\begin{array}{l}\text { Clstr Height } \\
\text { (bottom) (m) }\end{array}$ \\
\hline$\Leftrightarrow 4.5$ & $E$ & 01 & 7556 & $E$ & 15 & 14 \\
\hline 270 & 1 & $c 1$ & T严 65 & $\varepsilon$ & 14 & 14 \\
\hline 300 & $E$ & $\mathrm{ca}$ & $t^{2} 24$ & $E$ & 9 & 9 \\
\hline 210 & D & $i 2$ & $T / 3$ & $E$ & 12 & 12 \\
\hline 115 & $D$ & $i 2$ & 165 & $-\ell$ & 1 & 11 \\
\hline 255 & D & $i 2$ & 713 & $I$ & 7 & 7 \\
\hline 265 & $D$ & $d 4$ & 770 & $E$ & 8 & 198 \\
\hline 230 & $D$ & $d 5$ & 170 & $E$ & 1 & 10 \\
\hline 160 & D & 25 & 170 & $E$ & स 9 & 正 \& \\
\hline 162 zef & D & $d 5$ & .70 & $E$ & 8 & 8 \\
\hline 100 & D) & $\mathrm{Cr}$ & 169 & 5 & 15 & 15 \\
\hline 285 & $D$ & d4 & T69 & $\epsilon$ & $\$ 5 / 0$ & 10 \\
\hline 100 & $D$ & $d 4$ & $T 69$ & $E$ & $\|$ & 11 \\
\hline 31 & $P$ & d 3 & 769 & $E$ & 11 & 11 \\
\hline 45 & $D$ & d 3 & 169 & $E$ & 11 & f \\
\hline 135 & L & $; 9$ & 783 & $E$ & 7 & 7 \\
\hline \multicolumn{5}{|c|}{ nchude count from next pg) } & \multicolumn{2}{|l|}{ Total \# Trees: } \\
\hline \multicolumn{5}{|l|}{ H Lonersi: } & & \multirow{4}{*}{$\begin{array}{l}\text { Tally every butterfly of } \\
\text { each tope that you see } \\
\text { throughout the sitc. }\end{array}$} \\
\hline \multicolumn{5}{|l|}{ \# Sunners: || } & Tallye & \\
\hline \multirow{2}{*}{\multicolumn{5}{|c|}{$\begin{array}{l}\text { \# Fliers: }|||| \mid \\
\text { | Grounders (live): }\end{array}$}} & througs & \\
\hline \multirow{2}{*}{\multicolumn{4}{|c|}{$\begin{array}{l}\text { f Grounders (live): } \\
\text { Grand Totul? }\end{array}$}} & & & \\
\hline & & trame & of all chuster & red buttegfiks pia & o bowers samers $\beta i$ & ars estej \\
\hline
\end{tabular}

\begin{tabular}{|l|l|}
\hline A Mating Menarehs: & \\
\hline \# Dead Monarehs: & \\
\hline Other Notes: & \\
\hline
\end{tabular}




\begin{tabular}{|c|c|c|c|c|c|c|}
\hline $\begin{array}{l}\text { Monarehs } \\
\text { Clustered }\end{array}$ & $\begin{array}{l}\text { Zone } \\
\text { (ex: A) }\end{array}$ & $\begin{array}{c}\text { Cell } \\
(\mathrm{ex:} a 2)\end{array}$ & Tree "II & Tree Speeies & $\begin{array}{l}\text { Cluster Height } \\
\text { (top) (m) }\end{array}$ & $\begin{array}{l}\text { Clstr Height } \\
\text { (bottom) (m) }\end{array}$ \\
\hline 115 & C & $; 9$ & 783 & $\hat{c}$ & 9 & 9 \\
\hline 700 & c & i9 & 183 & $E$ & $\neq 10$ & 69 \\
\hline 150 & $\mathrm{H}$ & h8 & $T 94$ & $\bar{E}$ & 15 & 14 \\
\hline 75 & $H$ & h8 & T94 & $E$ & 14 & 14 \\
\hline 170 & $H$ & 98 & Ta4 & $E$ & 13 & 13 \\
\hline 75 & $\mathrm{H}$ & 98 & T94 & $E$ & 13 & 13 \\
\hline 7 & & & & & & \\
\hline & & & & & & \\
\hline & & & & & & \\
\hline & & & & & & \\
\hline & & & & & & \\
\hline & & & & & & \\
\hline & & & & & & \\
\hline & & & & & & \\
\hline & & & & & & \\
\hline  & & & & & & \\
\hline & & & & & & \\
\hline & & & & & & \\
\hline & & & & & & \\
\hline 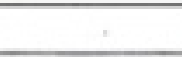 & & & & & & \\
\hline & & & & & & \\
\hline & & & & & & \\
\hline & & & & & & \\
\hline & & & & & & \\
\hline & & & & & & \\
\hline & & & & & & \\
\hline & & & & & & \\
\hline & & & & & & \\
\hline & & & & & & \\
\hline & & & & 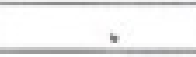 & & \\
\hline & & & & & & \\
\hline & & & & & & \\
\hline & & & & & & \\
\hline
\end{tabular}

Please add any cownts an this page to the total tally on the prewious page. 


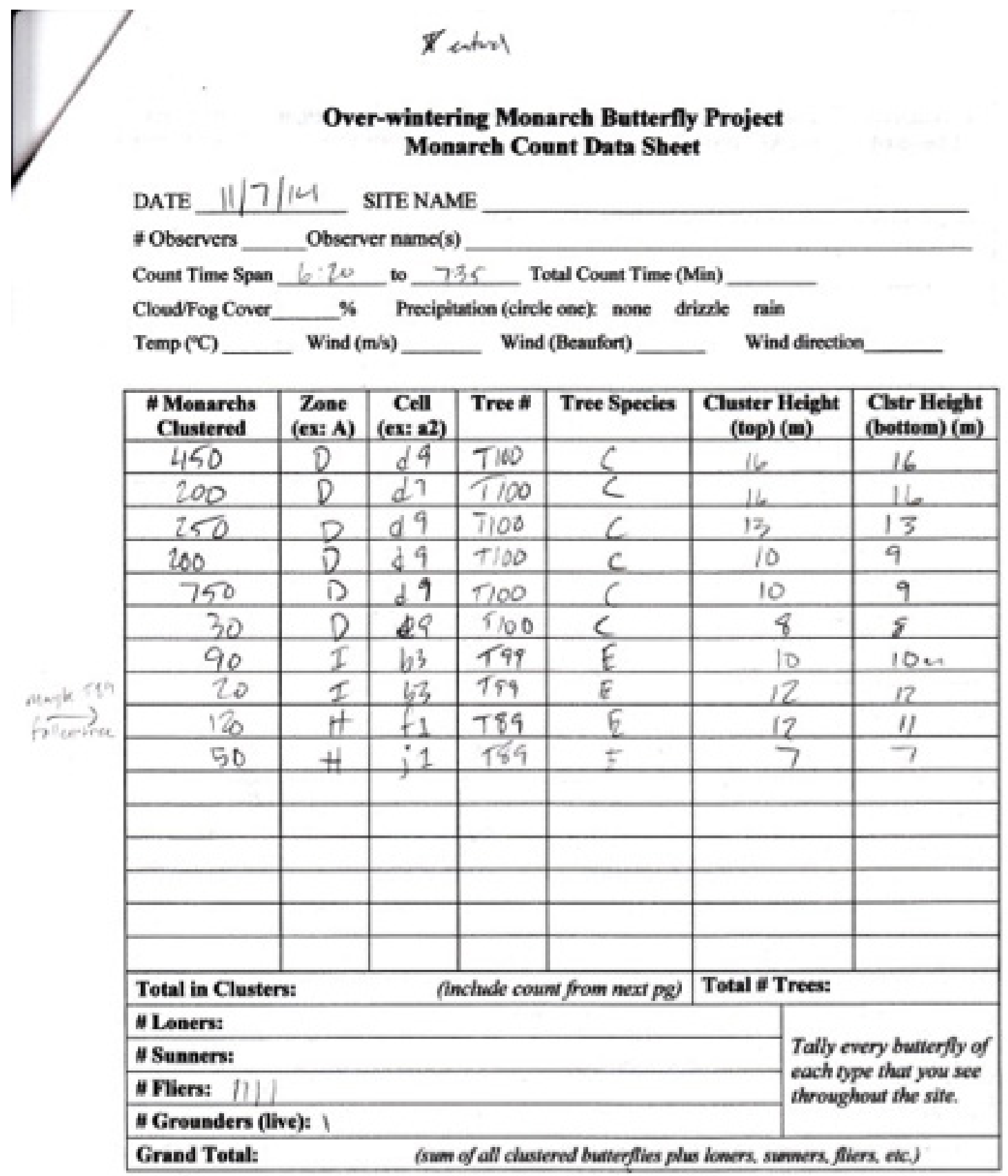

\begin{tabular}{|l|l|}
\hline \# Mating Monarehs: & \\
\hline H Dead Menarehs: & \\
\hline Other Notest & \\
\hline Ull ov th 9,54100 \\
\hline \\
\hline
\end{tabular}




$$
\text { Xestered }
$$

\section{Over-wintering Monarch Butterfly Project Monareh Count Data Sheet}

DATE $11-8-141$ STE NAME Pisme

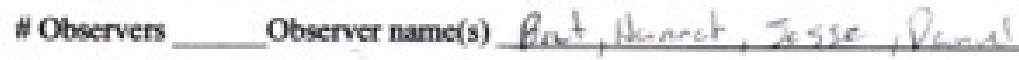

Count Time Span 6:15 to 5:002 Total Count Time (Min)

Cloud/Fog Cover low \% Procipitation (circle one): none) drizsle rain

Temp $\left({ }^{\circ} \mathrm{C}\right) \mathrm{B}, 2$ Wisd $(\mathrm{m} / \mathrm{s}) \_$Wind (Beaufort) $\_$Wind direction 0

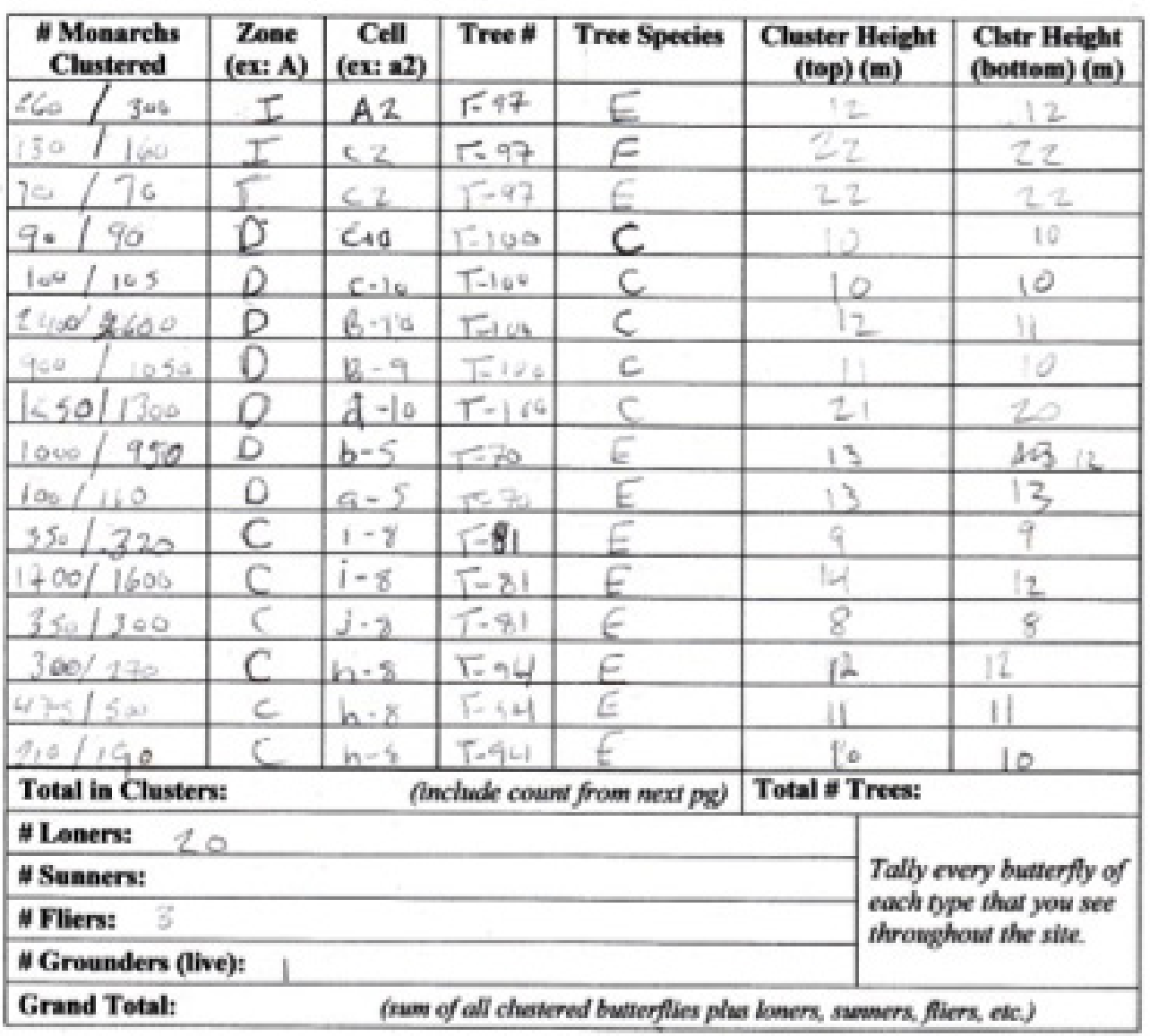

\begin{tabular}{|l|l|}
\hline Wating Monarchs: & \\
\hline W Dead Monarchs: & \\
\hline Other Netes: & \\
\hline
\end{tabular}




\section{2 enturet \\ Over-wintering Monarch Butterfly Project Monarch Count Data Sheet}

DATE II) $8 / 14$ SITE Name Pirno

"Observers 4 Observer name(s) Buelt, Howneh, Jesse, Dand

Count Time Span 6:20n to 8 m Total Count Time (Min) Tan so

Cloud Fog Cover $100 \%$ Procipitation (cirele one): (none) drizzle rain

Temp (CC) 13.2 Wind (m/s) $\mathcal{O}$ Wind (Beaufort) ___ Wind diroction_a $\mathrm{K} /$

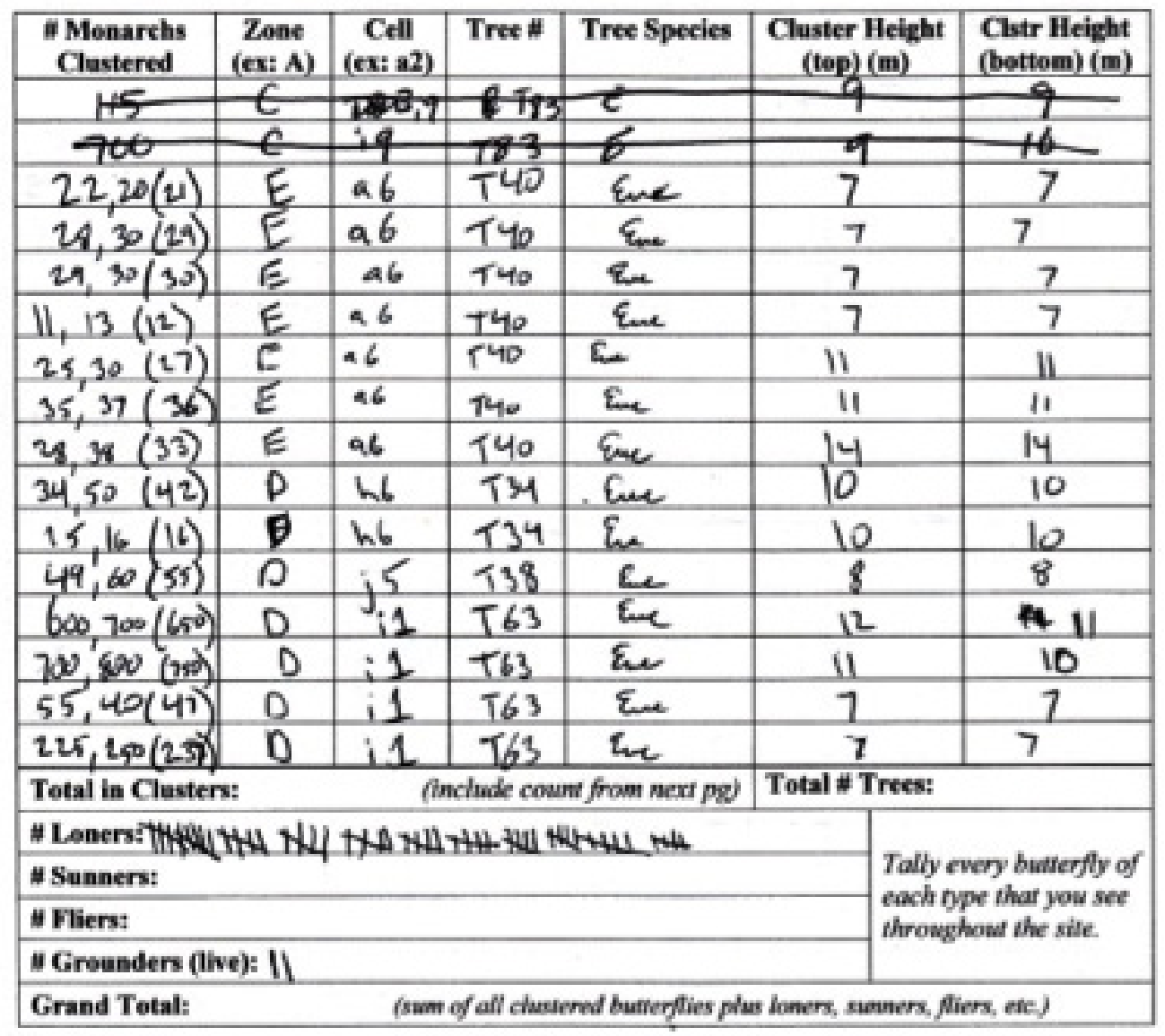

\begin{tabular}{|c|c|}
\hline " Mating Monarehs: & \\
\hline in Dead Menarchs: & \\
\hline Other Notes: & my fialos wt waining. Gy? \\
\hline
\end{tabular}




\begin{tabular}{|c|c|c|c|c|c|c|}
\hline $\begin{array}{l}\text { "Monarehs } \\
\text { Clustered }\end{array}$ & \begin{tabular}{|c|} 
Zone \\
(ex: A)
\end{tabular} & $\begin{array}{c}\text { Cell } \\
(\text { (ex: a2) }\end{array}$ & Tree "\# & Tree Species & $\begin{array}{l}\text { Cluster Height } \\
\text { (top) (m) }\end{array}$ & $\begin{array}{l}\text { Clstr Height } \\
\text { (bottom)(m) }\end{array}$ \\
\hline$(24,20,20(2)$ & 0 & $h 1$ & $T 63$ & Enc & 8 & 8 \\
\hline $350,300(3 \mathrm{~m})$ & 0 & C2 & $T_{69}$ & Ene & 24 & 23 \\
\hline $100,130(45)$ & $D$ & c1 & 169 & Ev & 23 & 23 \\
\hline $28,35(32)$ & 0 & e2 & T69 & Es & n & "1 \\
\hline $110,90(100)$ & D & $d z$ & $T 70$ & Ene & II & $i$ \\
\hline $85,66(75)^{\prime}$ & $n$ & $d_{3}$ & 770 & \&e & 10 & 10 \\
\hline 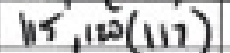 & D & $d 3$ & 170 & Ene & 10 & 10 \\
\hline $200,150(n n)$ & D & du & 720 & En & 11 & 11 \\
\hline $40,55(38)$ & 0 & $\mathrm{c4}$ & 570 & Ence & 9 & 9 \\
\hline $50,6(55)$ & C & Shlo & collim & Ene & 7 & 7 \\
\hline & & & & & & \\
\hline & & & & & & \\
\hline & & & & & & \\
\hline & & & & & & \\
\hline & & & & & & \\
\hline & & & & & & \\
\hline & & & & & & \\
\hline & & & & & & \\
\hline & & & & & & \\
\hline & & & & & & \\
\hline & & & & & & \\
\hline & & & & & & \\
\hline & & & & & & \\
\hline & & & & & & \\
\hline & & & & & & \\
\hline & & & & & & \\
\hline & & & & & & \\
\hline & & & & & & \\
\hline & & & & - & & \\
\hline & & & & & & \\
\hline & & & & & & \\
\hline
\end{tabular}

Please add any counts an this page to the total tally on the previous page. 


\section{REntad. \\ Over-wintering Monarch Butterfly Project Monareh Count Data Sheet}

DATE $1+q 14$ STIE Name Pisma

\#Observers 4 Observer name(s) Dened, Hannah, Nacule, Emily. Count Time Span 6:15 to \& Total Count Time (Min).

CloudFog Cover las \% Procipitation (circle one): (none) drisele rain

Temp ("C) 12.2 Wind (m/s) $\quad 0.2$ Wind (Beaufort) ___ Wind diroction_E

\begin{tabular}{|c|c|c|c|c|c|c|c|}
\hline $\begin{array}{l}\text { Wonarchs } \\
\text { Clustered }\end{array}$ & $\begin{array}{c}\text { Zone } \\
(\mathrm{ex:}: \mathrm{A})\end{array}$ & $\begin{array}{c}\text { Cell } \\
\left(\mathrm{ex} ; \mathrm{x}_{2}\right)\end{array}$ & Tree H & Tree Species & \multirow{2}{*}{\multicolumn{2}{|c|}{$\begin{array}{c}\begin{array}{c}\text { Cluster Height } \\
\text { (tep) }(\mathbf{m})\end{array} \\
\text { i? }\end{array}$}} & $\begin{array}{l}\text { Clstr Height } \\
\text { (bottom) }(m)\end{array}$ \\
\hline $30^{\circ} / 27$ & $I$ & $A Z$ & Tiq & $E$ & & & 12 \\
\hline $110 / 140$ & $I$ & $\Delta 2$ & T99 & $E$ & \multicolumn{2}{|l|}{13} & 13 \\
\hline $180 / 170$ & $I$ & $\mathrm{Cz}$ & $T 79$ & $E$ & \multicolumn{2}{|c|}{22} & 22 \\
\hline $90 / 70$ & $I$ & $\mathrm{Cl}_{2}$ & Trq & $E$ & \multicolumn{2}{|c|}{22} & 22 \\
\hline$6 0 \longdiv { 5 0 }$ & $I$ & $C_{2}$ & 199 & $E$ & \multicolumn{2}{|l|}{22} & 22 \\
\hline$1 0 0 \longdiv { 8 0 }$ & 0 & $\mathrm{Cll}$ & Tion & C & \multicolumn{2}{|l|}{10} & ic \\
\hline $400 / 150$ & 0 & $\mathrm{Clu}$ & Titu & C & \multicolumn{2}{|l|}{12} & a \\
\hline $55 / 50$ & 0 & Bin & Tioe & $c$ & \multicolumn{2}{|l|}{ u } & to \\
\hline $625 / 650$ & D & $\mathrm{Bq}$ & Tina & $c$ & \multicolumn{2}{|l|}{$\mu$} & 10 \\
\hline $1600 / 1500$ & 0 & dio & Ties & c & \multicolumn{2}{|l|}{21} & 20 \\
\hline $190 / 210$ & $D$ & duc & The & $c$ & \multicolumn{2}{|l|}{$z_{1}$} & 20 \\
\hline $200 / 160$ & $C$ & $h-8$ & Ta4 & $E$ & \multicolumn{2}{|c|}{10} & 10 \\
\hline $500 / 550$ & C & $h-8$ & $T 94$ & $E$ & \multicolumn{2}{|l|}{11} & 11 \\
\hline $350 / 350$ & C & $h-z$ & T94 & $E$ & \multicolumn{2}{|l|}{$n$} & 12 \\
\hline $300 / 250$ & c. & $i-7$ & 181 & E & \multicolumn{2}{|l|}{8} & 8 \\
\hline$2 5 8 \longdiv { 2 9 0 }$ & $c$ & $1-9$ & $\Gamma 81$ & $E$ & \multicolumn{2}{|l|}{ ร } & 9 \\
\hline \multicolumn{2}{|c|}{ Total in Clusters: } & \multicolumn{3}{|c|}{ (include count from next pg) } & \multicolumn{3}{|c|}{ Total i Trees: } \\
\hline \multicolumn{5}{|l|}{ \# Loners: } & & \multirow{4}{*}{\multicolumn{2}{|c|}{$\begin{array}{l}\text { Tally every bualerfly of } \\
\text { each type that you see } \\
\text { throwghout the site. }\end{array}$}} \\
\hline \multicolumn{6}{|l|}{ " Sunners: } & & \\
\hline \multicolumn{5}{|l|}{ " Fliers: 1} & & & \\
\hline \multicolumn{5}{|c|}{ (n Grounders (live): } & & & \\
\hline \multicolumn{2}{|l|}{ Grand Total: } & \multicolumn{6}{|c|}{ (stum of all clustered butrerflies phas loners. sumern. filers, etc) } \\
\hline
\end{tabular}

\begin{tabular}{|l|l|}
\hline W Mating Monarchs: & \\
\hline W Dead Monarehs: & \\
\hline Other Notes: & \\
\hline
\end{tabular}




\begin{tabular}{|c|c|c|c|c|c|c|}
\hline $\begin{array}{l}\text { Monarchs } \\
\text { Clustered }\end{array}$ & $\begin{array}{c}\text { Zone } \\
(\mathbf{e x :} \text { A) }\end{array}$ & $\begin{array}{c}\text { Cell } \\
\text { (ex: az) }\end{array}$ & Tree * & Tree Species & $\begin{array}{l}\text { Cluster Height } \\
\text { (top) }(m)\end{array}$ & $\begin{array}{l}\text { Clstr Height } \\
\text { (bottom) (m) }\end{array}$ \\
\hline $1400 / 1200$ & $C$ & $i-\gamma$ & $T^{-81}$ & $E$ & 12 & 12 \\
\hline 80170 & c & $e-q$ & $T-92$ & $E$ & 9 & 9 \\
\hline 45.1500 & $D$ & Q -4 & $T=69$ & $E$ & Le & 20 \\
\hline & & & & & & \\
\hline & & & & & & \\
\hline & & & & & & \\
\hline & & & & & & 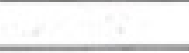 \\
\hline & & & & & 76 & \\
\hline & & & & 5 & & $\overline{1}$ \\
\hline & & & & & & \\
\hline & & & & & & \\
\hline & & & & & & \\
\hline & & & & & & \\
\hline & & & & - & & \\
\hline & & & & & & \\
\hline & & & & & & \\
\hline & & & & & & \\
\hline & & & & & & \\
\hline & & & & & & 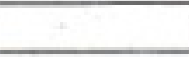 \\
\hline & & & & & & \\
\hline & & & & & & \\
\hline & & & & & & \\
\hline & & & & & & \\
\hline & & & & & & \\
\hline & & & & & & \\
\hline & & & & & & \\
\hline & & & & & 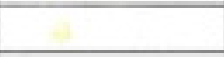 & \\
\hline & & & & & & \\
\hline & & & & & & \\
\hline & & & & 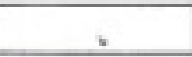 & & \\
\hline & & & & & & \\
\hline & & & & & & \\
\hline & & & & & & \\
\hline
\end{tabular}

Please add any counts on this page to the total tally on the previous page. 


\section{Entrow. \\ Over-wintering Monarch Butterfly Project Monareh Count Data Sheet}

DATE $11 / 9 / 14$ SITE NAME

\# Observers 12 Observer name(s) Enily, Nick

Count Time Span 6:20 to 7:57 Total Count Time (Min)

Cloudfog Cover $100 \%$ Precipintion (circle one): drizzle min

Temp ( $(\mathrm{C}) 12.2$ Wind $(\mathrm{m} / \mathrm{s}) .2$ Wind (Beaufort) ___ Wind direction $\mathrm{S}$

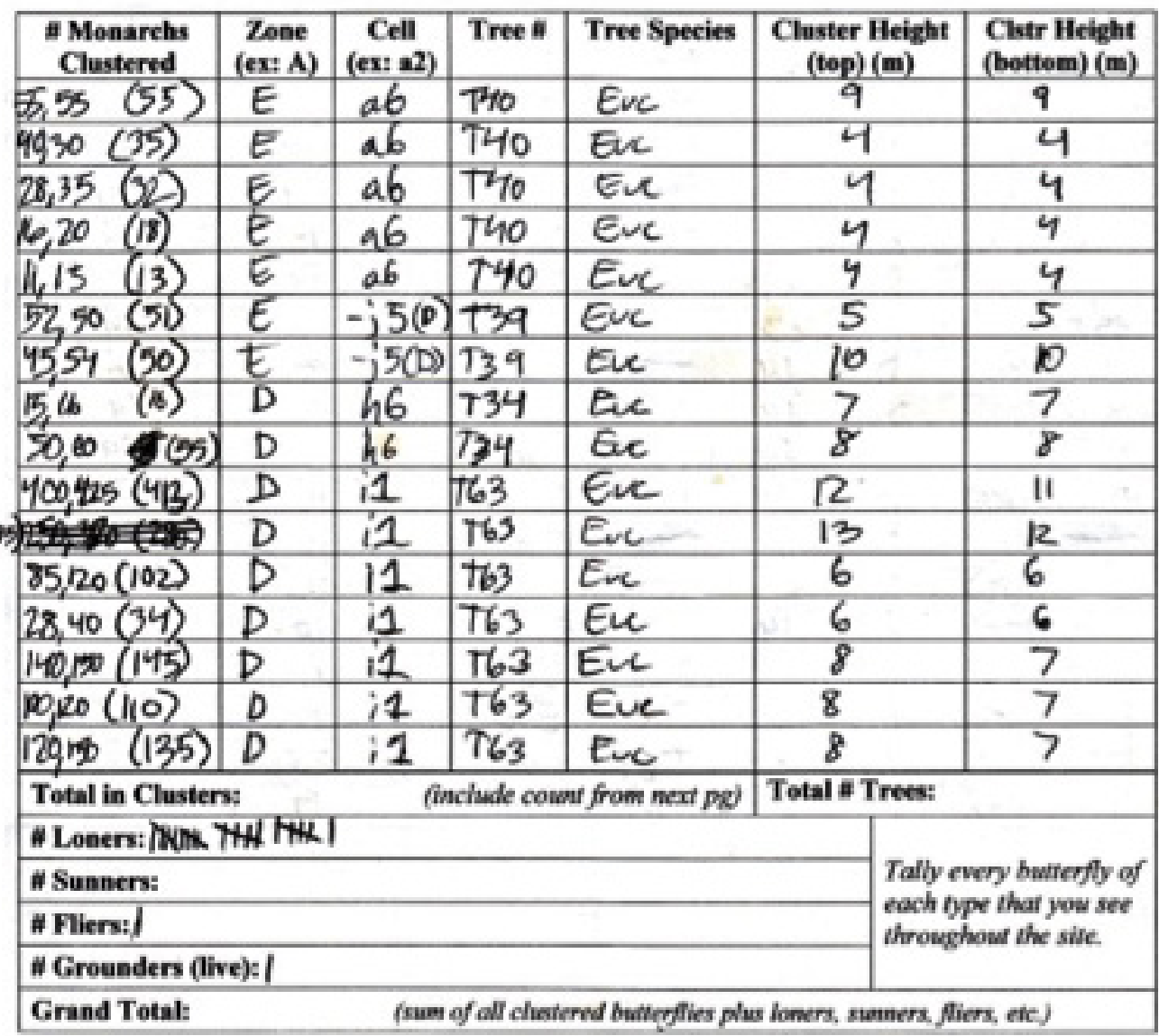

\begin{tabular}{|l|l|}
\hline W Mating Monarehs: & \\
\hline Dead Monarehs: & \\
\hline Other Notes: & \\
\hline
\end{tabular}

I dryonfly 


\begin{tabular}{|c|c|c|c|c|c|c|}
\hline $\begin{array}{l}\text { Monarebs } \\
\text { Clustered }\end{array}$ & $\begin{array}{c}\text { Zone } \\
\text { (ex: A) }\end{array}$ & $\begin{array}{c}\text { Cell } \\
(\mathbf{e x :}: 22)\end{array}$ & Tree \# & Tree Species & $\begin{array}{l}\text { Cluster Height } \\
\text { (top) (m) }\end{array}$ & $\begin{array}{l}\text { Cstr Height } \\
\text { (bottom) (m) }\end{array}$ \\
\hline $80,80(80)$ & D & if & T63 & Euc & 6 & 6 \\
\hline $150,200(175)$ & D & if & T63 & Euc & II & to \\
\hline $16,16(16)$ & D & 291 & T63 & Euc & 8 & 8 \\
\hline $60,83(72)$ & $D$ & $e 3$ & $T 70$ & Ex & II & 10 \\
\hline $140,115(127)$ & D & $e^{3}$ & 170 & Eve & 11 & II \\
\hline $30,290(35)$ & D & e3 & 769 & Euc & 11 & 11 \\
\hline $120,120(120)$ & D & e3 & $T 70$ & Eur & 10 & 10 \\
\hline $100 / 100(100)$ & D & e3 & 170 & Euc & 13 & $R$ \\
\hline $100,100(170)$ & D & $c^{3}$ & 170 & EuC & 13 & 13 \\
\hline $100,110 \quad(105)$ & D & e3 & $T 70$ & Eve & 13 & 13 \\
\hline 160,170 (165) & $D$ & $e 3$ & $770^{\circ}$ & Eu & 14 & 13 \\
\hline $4550(n>)$ & D & $e 3$ & 170 & $E_{x}$ & 14 & 14 \\
\hline $18,16(17)$ & $D$ & $e^{3}$ & $T 70$ & Euc & 14 & 14 \\
\hline $125145(135)$ & D & .3 & $T \pi 0$ & Euc & 14 & 14 \\
\hline $45,50(47)$ & $D$ & e3 & T70 & Euc & 14 & 14 \\
\hline $\mid 10,110(\mathbf{b})$ & D & 24 & 170 & Eu & 10 & 10 \\
\hline $25,32(28)$ & D & $d^{4}$ & T70 & Euc & 9 & 9 \\
\hline $35,40(37)$ & D & 24 & 770 & Euc & 9 & 9 \\
\hline $90,120(105)$ & D & $d^{4}$ & $T 70$ & Evc & II & II \\
\hline $2075(72)$ & D & 24 & T70 & Eve & 11 & 11 \\
\hline $60,50(5 n)$ & D & 24 & 770 & Euc & R & 12 \\
\hline $180,170(175)$ & D & $d^{4}$ & 770 & Eve & 10 & 9 \\
\hline $230,210(235)$ & D & 24 & $T>0$ & Eve & 10 & 9 \\
\hline $80,80(80)$ & D & d4 & $T 70$ & Eve & 10 & 9 \\
\hline $175.180(177)$ & $D$ & $d 4$ & $T 69$ & Euc & 20 & 20 \\
\hline $35,50(43)$ & $D$ & $d 4$ & $T 69$ & Euc & 17 & 17 \\
\hline & & & & & & \\
\hline & & & & & & \\
\hline & & & & & & \\
\hline & & & & - & & \\
\hline & & & & & & \\
\hline & & & & & & \\
\hline & & & & & & \\
\hline
\end{tabular}

Please add any counts on this page to the total tally on the previous page. 


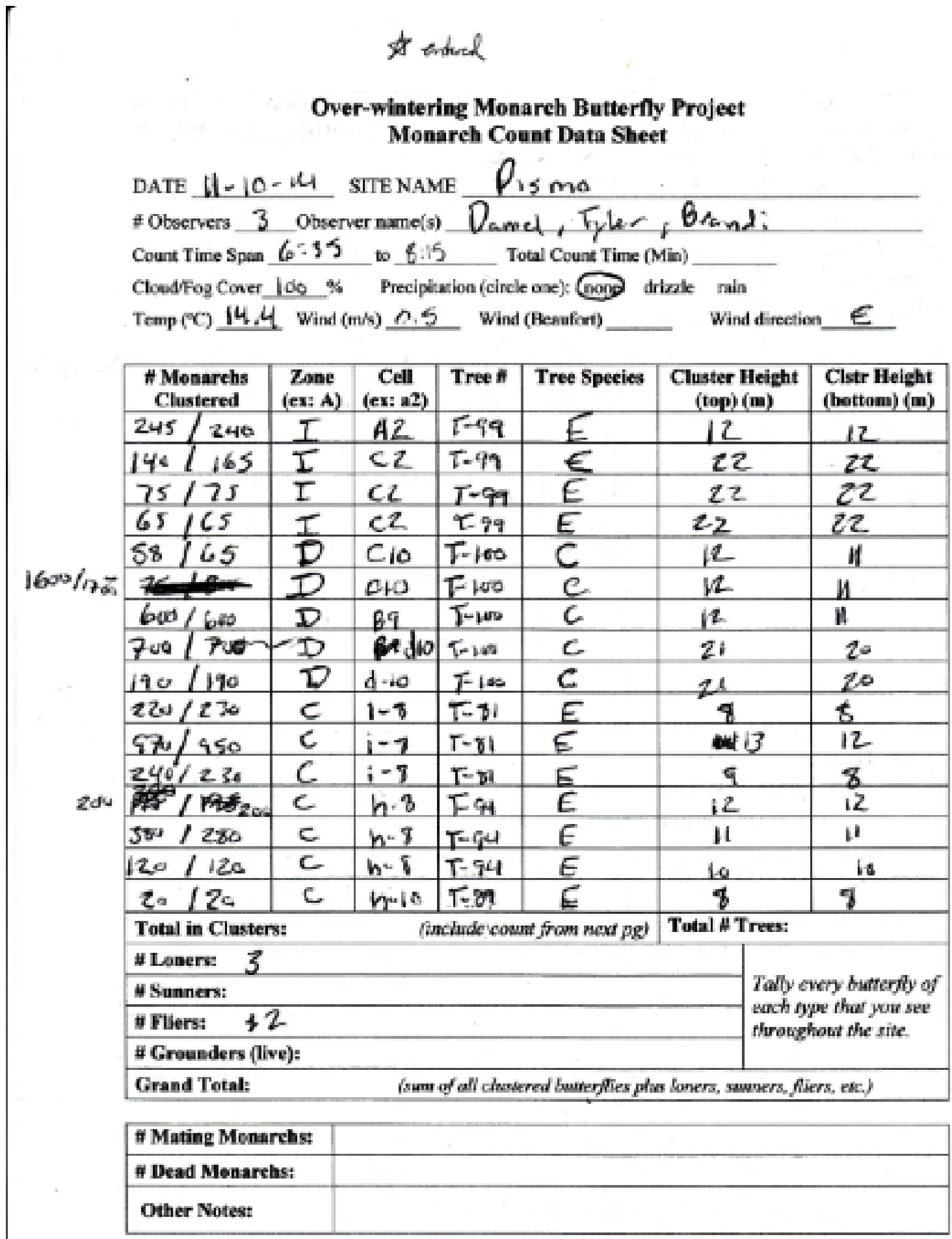




\begin{tabular}{|c|c|c|c|c|c|c|}
\hline $\begin{array}{l}\text { Wonarebs } \\
\text { Clustered }\end{array}$ & $\begin{array}{c}\text { Zone } \\
\text { (ex: A) }\end{array}$ & $\begin{array}{c}\text { Cell } \\
(\mathbf{e x t} a 2)\end{array}$ & $\begin{array}{r}\text { Tree " } \\
70\end{array}$ & Tree $\mathrm{Species}$ & $\begin{array}{l}\text { Cluster Height } \\
\text { (tep) (m) }\end{array}$ & $\begin{array}{l}\text { Clstr Height } \\
\text { (bottem) (m) }\end{array}$ \\
\hline 6a 1650 & $D$ & $b-5$ & $T=$ & $E$ & 10 & 10 \\
\hline $30^{\circ} / 320$ & 0 & $E-4$ & $T=90$ & $\vec{E}$ & 11 & u \\
\hline 250135 & 0 & d. 4 & $T-62$ & $E$ & 22 & $2 z$ \\
\hline $300 / 360$ & $P$ & $c-5$ & $T-69$ & $E$ & 22 & 22 \\
\hline $350 / 400$ & 0 & $i=1$ & $T .63$ & 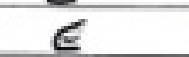 & 12 & 12 \\
\hline $35 / 13 \%$ & 0 & $i-2$ & $T-63$ & $E$ & H & 11 \\
\hline $350 / 320$ & $D$ & $i-2$ & $T \cdot 63$ & $E$ & 8 & 7 \\
\hline $450 / 50^{\circ}$ & $E$ & $G=4$ & $T=42$ & $E$ & 9 & 9 \\
\hline $10 / 130$ & $E$ & $5-4$ & $T-42$ & $E$ & 16 & 16 \\
\hline $5=145$ & 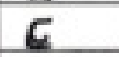 & $9 \cdot 4$ & T.un & E & 16 & 16 \\
\hline $250 / 260$ & $E$ & $a \cdot-4$ & $T_{42}$ & $E$ & 21 & 21 \\
\hline $60 / 60$ & $E$ & 6.6 & $T=40$ & $E$ & 8 & 8 \\
\hline $40 / 50$ & $E$ & $b-6$ & $T=40$ & $E$ & 12 & 12 \\
\hline 1 & $E$ & $6-6$ & Eve & $E$ & & \\
\hline & & & & & 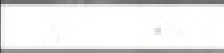 & \\
\hline & & & & & & \\
\hline & & & & & & \\
\hline & & & & & & \\
\hline & & & & & & \\
\hline & & & & & & \\
\hline & & & & & & \\
\hline & & & & & & \\
\hline & & & & & & \\
\hline & & & & & & \\
\hline & & & & & & \\
\hline & & & & & & \\
\hline & & & & & & \\
\hline & & & & & & \\
\hline & & & & & & \\
\hline & & & & & & \\
\hline & & & & - & & \\
\hline & & & & & & \\
\hline & & & & & & \\
\hline & & & & & & \\
\hline Please add any. & ints o & spag & le to & $y$ on the pr & us page. & \\
\hline
\end{tabular}




\section{Over-wintering Monareh Butterfly Project Monarch Count Data Sheet}

DATE $11 / 11 / 4$ SITE NAME

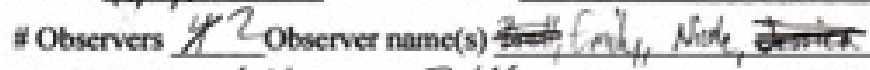

Coumt Time Span 6:21

to $7: 46$

Total Count Time (Min) 85

Cloud/Fog Cover $80 \%$ Procipitation (eircle one): 1 drizzle nin Temp (cC) 186 Wind (m/s) 0 Wind (Beaufort) 0 Wind direction

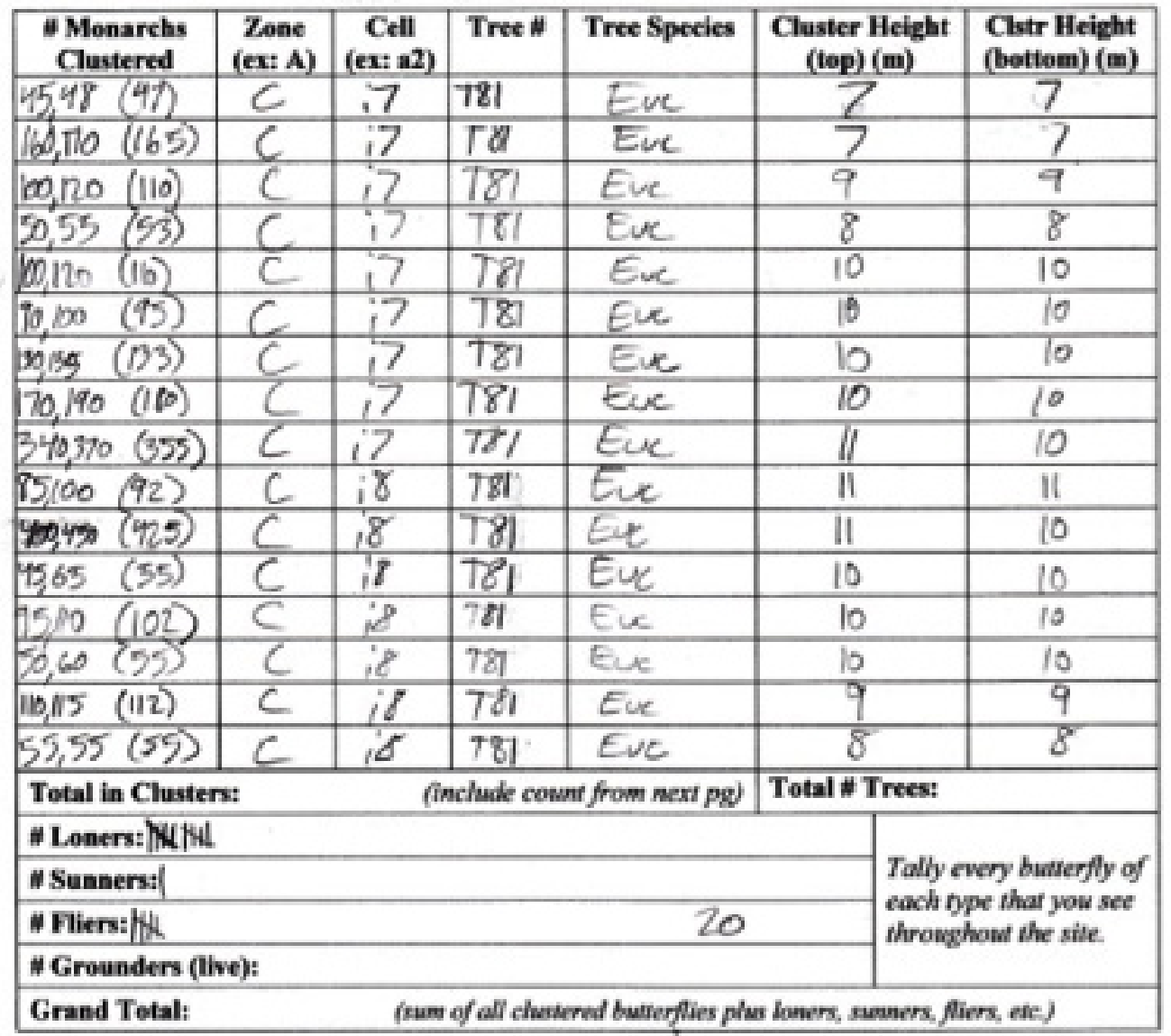

\begin{tabular}{|l|l|}
\hline W Mating Monarchs: & \\
\hline W Dead Monarchs: & \\
\hline Other Notes: & \\
\hline
\end{tabular}




\begin{tabular}{|c|c|c|c|c|c|c|}
\hline $\begin{array}{l}\text { "Monarehs } \\
\text { Clustered }\end{array}$ & $\begin{array}{c}\text { Zone } \\
\text { (ex: A) }\end{array}$ & $\underset{\left(\operatorname{cxs} \mathbf{n}^{2}\right)}{\text { Cenll }}$ & Tree "I & Tree Species & $\begin{array}{c}\text { Cluster Height } \\
\text { (top) (m) }\end{array}$ & $\begin{array}{l}\text { Clstr Height } \\
\text { (bottom) (m) }\end{array}$ \\
\hline $155,160 \quad(152)$ & c & $i 8$ & $T 8$ & Ex & 9 & 8 \\
\hline $90,46(43)$ & C & it & T81 & Ex & 8 & 8 \\
\hline 0,160 (155) & C & 8 & $T 81$ & Euc & 8 & 8 \\
\hline $170156(128)$ & C & 18 & 781 & Euc & 8 & 7 \\
\hline $190,190(190)$ & C & is & 781 & Eve & 7 & 6 \\
\hline $45,45(45)$ & C & if & 781 & Eu & 6 & 6 \\
\hline $50,60(55)$ & C & it & 781 & Evc & 7 & 7 \\
\hline $5.75(63)$ & C & $i 8$ & 781 & Eu & 6 & 6 \\
\hline $15,50(47)$ & C & $i 8$ & 781 & Eue & 6 & 6 \\
\hline $60,75(70)$ & C & 8 & $T 81$ & Eu & 7 & 7 \\
\hline $150,120(160)$ & C & h7 & Taq & Euc & 11 & II \\
\hline $160,175(168)$ & $C$ & h7 & $T 94$ & Eve & II & II \\
\hline $10,110(115)$ & C & h7 & 794 & $E_{V C}$ & II & 10 \\
\hline $7080 \quad(75)$ & C & in & $T 94$ & Exc & 10 & 9 \\
\hline $00,200 \quad(170)$ & C & h8 & $T M$ & Ex & 9 & 9 \\
\hline $160,175(168)$ & C & 48 & $T 94$ & Euc. & 9 & 9 \\
\hline $55,60(57)$ & C & $h_{B}$ & 194 & Euc & 9 & 9 \\
\hline $95,120(112)$ & C & 48 & 794 & Euc & 9 & 9 \\
\hline $40,60(50)$ & C & hr & 794 & Eve & 10 & 10 \\
\hline $30,40 \quad(35)$ & c & ho & 794 & Euc & 8 & 8 \\
\hline 40,70 (5) & C & 18 & $T q_{4}$ & Euc & 8 & 8 \\
\hline $45, \%(47)$ & C & 18 & T94 & Evc & 8 & 8 \\
\hline $55,70(63)$ & C & hi & 194 & Eue & 9 & 9 \\
\hline & & & & & & \\
\hline & & & & & & \\
\hline & & & & & & \\
\hline & & & & & & \\
\hline & & & & & & \\
\hline & & & & & & \\
\hline & & & & . & & \\
\hline & & & & & & \\
\hline & & & & & & \\
\hline
\end{tabular}




\section{Over-wintering Monarch Butterfly Project Monarch Count Data Sheet}

DATE $\perp 1 / 11 / 14$ SITE NAME

"Observers 4 Observer name(s) Bnett, Emily, ofevie, Jessica

Count Time Span 6:21 to 7:46

Total Count Time (Min) 85

CloudFog Cover $80 \%$ Procipitation (circle one): drizzle rain

Temp (CC) 18.6 Wind ( $\mathrm{m} / \mathrm{s}) \_$Wind (Beaufort) $0 \quad$ Wind diretion.

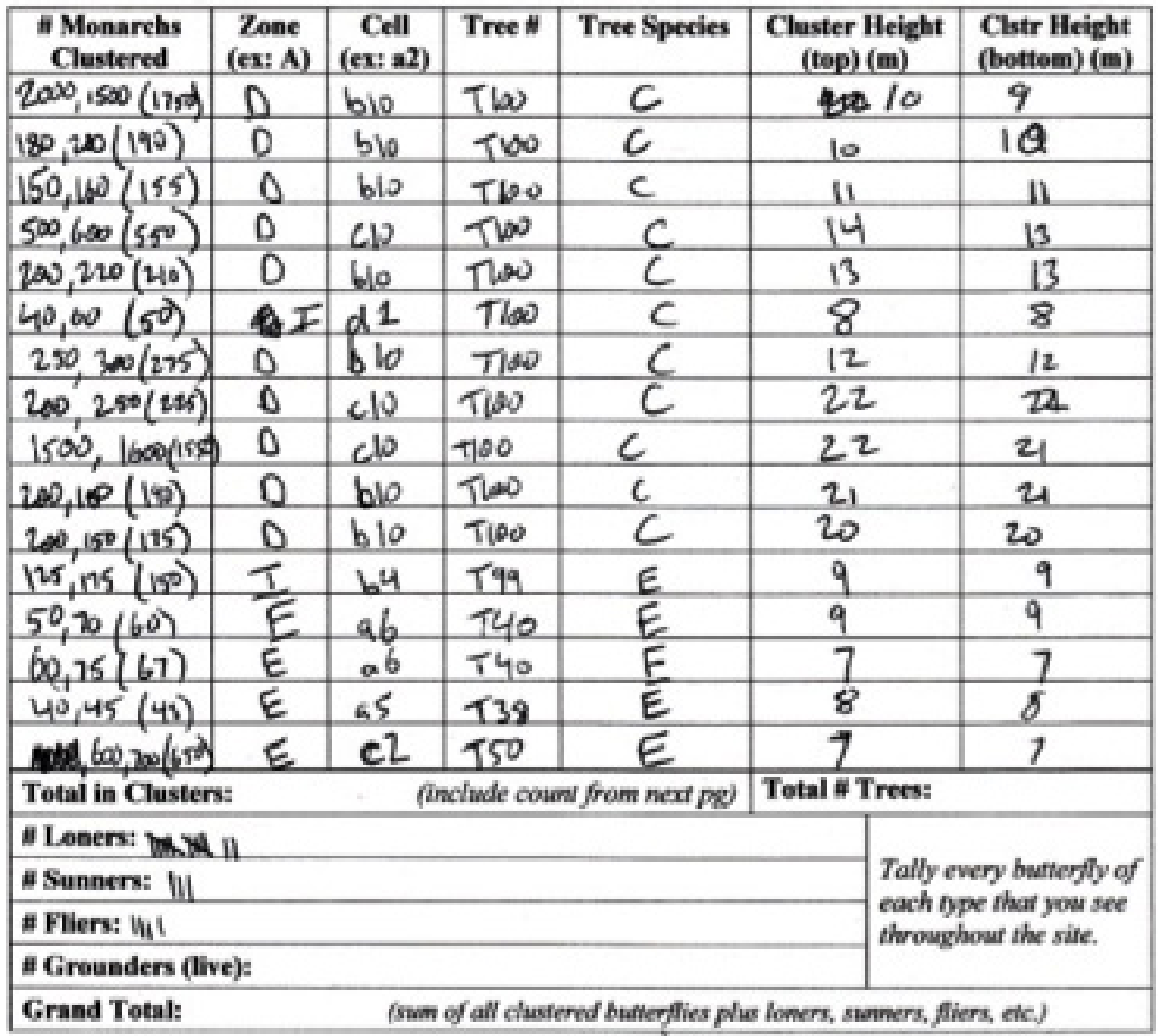

\begin{tabular}{|l|l|}
\hline II Mating Menarehs: & \\
\hline inead Monarehs: & \\
\hline Other Notes: & \\
\hline
\end{tabular}

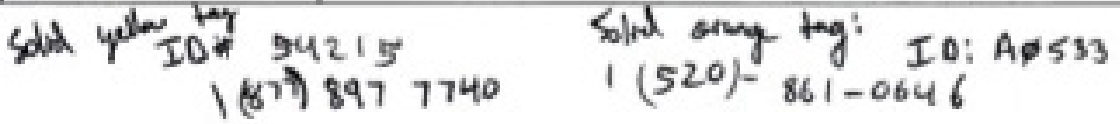




\begin{tabular}{|c|c|c|c|c|c|c|}
\hline $\begin{array}{l}\text { Wonarchs } \\
\text { Clustered }\end{array}$ & $\begin{array}{c}\text { Zone } \\
(\mathrm{ex}: \mathrm{A})\end{array}$ & $\begin{array}{c}\text { Cell } \\
(\mathbf{e x :}: 2)\end{array}$ & Tree in & Tree Species & $\begin{array}{l}\text { Cluster Height } \\
\text { (top) (m) }\end{array}$ & $\begin{array}{l}\text { Clstr Height } \\
\text { (bottom) (m) }\end{array}$ \\
\hline $120,10(110)$ & $E$ & C 1 & 159 & $E$ & 7 & 1 \\
\hline$(100)$ & $E$ & $c_{1}$ & $15 q$ & E & 1.1 & 10 \\
\hline$(100)$ & $E$ & $\mathrm{Cl}$ & TS9 & E & 11 & 10 \\
\hline$(80)$ & $E$ & $\mathrm{Cl}^{\prime}$ & TS9 & E & 11 & 10 \\
\hline$(250)$ & E D & 11 & T63 & E & 8 & 7 \\
\hline$(150)$ & P & is & $T 63$ & $E$ & 27 & 2 \\
\hline$(80)^{\prime}$ & $D$ & is & $T 63$ & $E$ & 7 & 7 \\
\hline $300,300(\pi)$ & 0 & $c^{2}$ & $T_{69}$ & E & 23 & 23 \\
\hline $150,170(167)$ & D & $\mathrm{Cl}^{2}$ & T69 & $E$ & 23 & 23 \\
\hline $50,50(50)$ & $D$ & h1 & $T 69$ & $E$ & 24 & 24 \\
\hline $200,200(200)$ & D & $d y$ & $T 70$ & $E$ & 12 & 12 \\
\hline $400,300(35)$ & 0 & 24 & $T 70$ & E & 10 & 10 \\
\hline $\left.200,10^{\circ}(11)^{\circ}\right)$ & D & $d y$ & 170 & E & 10 & 10 \\
\hline $30,40(x)$ & 0 & 24 & $T 70$ & E & 10 & 10 \\
\hline 200 & $I$ & b3 & 199 & $E$ & 10 & 10 \\
\hline 100 & $I$ & 63 & T44 & $E$ & 12 & 12 \\
\hline & & & & & & \\
\hline & & & & & & \\
\hline & & & & & & \\
\hline & & & & & & \\
\hline  & & & & & & \\
\hline & & & & & & \\
\hline & & & & & & \\
\hline & & & & & & \\
\hline & & & & & & \\
\hline & & & & & & \\
\hline & & & & & & \\
\hline & & & & & & \\
\hline & & & & & & \\
\hline & & & & & & \\
\hline & & & & $=$ & & \\
\hline & & & & & & \\
\hline & & & & & & \\
\hline & & & & & & \\
\hline
\end{tabular}

Please add any cownts an this page to the fotal fally on the previous page.

80554 - orange tag 


\section{Over-wintering Monarch Butterfly Project Monarch Count Data Sheet}

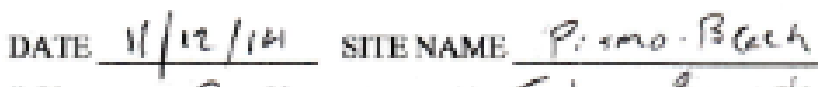

\# Observers 3 Observer name(s) Tyler, Brand: Jese

Count Time span 6 : $: 30$ to $7: 45$ Total Count Time (Mim) 75

Cloud Fog Cover_ $30 \%$ Precipitation (circle onc): enos drizzle / min

Temp $\left({ }^{\circ} \mathrm{C}\right) \quad 15 \cdot \overline{3}$ Wind (mis) $\quad 0.0$ Wind (Beaufort) ___ Wind direction



\begin{tabular}{|l|l|}
\hline \# Mating Monarchs: & \\
\hline \# Dead Monarehs: & \\
\hline Other Notes: & \\
\hline
\end{tabular}

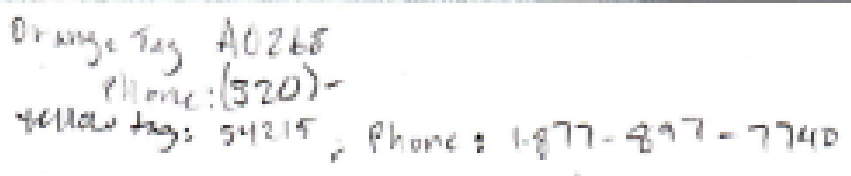




\begin{tabular}{|c|c|c|c|c|c|c|}
\hline $\begin{array}{l}\text { Monarehs } \\
\text { Clustered }\end{array}$ & $\begin{array}{c}\text { Zone } \\
\text { (ex: A) }\end{array}$ & $\begin{array}{c}\text { Cell } \\
(\mathrm{ex}: \mathrm{a} 2)\end{array}$ & Tree \# & Tree Species & $\begin{array}{l}\text { Cluster Height } \\
\text { (tep) (m) }\end{array}$ & $\begin{array}{l}\text { Cistr Height } \\
\text { (bottom) (m) }\end{array}$ \\
\hline $150 / 145$ & D & $b^{4}$ & $T b 9$ & $\Sigma$ & 70 & 20 \\
\hline $675 / 600$ & $D$ & $e^{4}$ & 570 & f & 11 & 10 \\
\hline $376 / 350$ & D & $e 4$ & $T 70$ & E & 9 & 9 \\
\hline $350 / 325$ & D & $e s$ & 170 & I & 9 & 9 \\
\hline $450 / 480$ & 0 & 64 & $T 10$ & E & 9 & 9 \\
\hline $100 / 75$ & $D$ & $a_{4}$ & 770 & $E$ & 9 & 9 \\
\hline $200 / 150$ & $c$ & is & 181 & $E$ & 8 & 8 \\
\hline $16 e^{2} / 1300$ & $C$ & 98 & $T 81$ & E & 12 & 10 \\
\hline $378 / 340$ & $c$ & 19 & $T 81$ & है & 8 & 8 \\
\hline $370 / 380$ & $c$ & $i 8$ & 791 & E & 11 & 11 \\
\hline$4 1 0 \longdiv { 3 8 0 }$ & c & is & 191 & $L$ & 4 & 9 \\
\hline $170 / 190$ & $C$ & h8 & 791 & $\varepsilon$ & 9 & 9 \\
\hline $250 / 240$ & c & 18 & 791 & 6 & 12 & 12 \\
\hline $120 \% 100$ & $C$ & hi & 791 & t & 10 & 10 \\
\hline & & & & & & \\
\hline & & & & & & \\
\hline & & & & & & \\
\hline & & & & & & \\
\hline & & & & & & \\
\hline & & & & & & \\
\hline & & & & & & \\
\hline & & & & & & \\
\hline & & & & & & \\
\hline & & & & & & \\
\hline & & & & & & \\
\hline & & & & & & \\
\hline & & & & & & \\
\hline & & & & & & \\
\hline & & & & & & \\
\hline & & & & & & \\
\hline & & & & $\checkmark$ & & \\
\hline & & & & & & \\
\hline & & & & & & \\
\hline
\end{tabular}




\section{Over-wintering Monarch Butterfly Project Monareh Count Data Sheet}

DATE $11 / 13 / / A$ SITE NAME

\# Observers 3 Observer name(s) Brett, $\mu_{\text {ool }}$, Emply

Count Time Span 6:36r to 8:05 Total Count Time (Min) ta 91

Cloud/Fog Cover $100 \%$ Precipitation (circle one)

Temp (C) 13.6 Wind $(\mathrm{m} / \mathrm{s}) \mathrm{O}$ Wind (Beaufort) 0 Wind dinection 새/t

\begin{tabular}{|c|c|c|c|c|c|c|c|c|}
\hline & $\begin{array}{l}\text { "Monarehs } \\
\text { Clustered }\end{array}$ & $\begin{array}{c}\text { Zone } \\
\text { (ex: A) }\end{array}$ & $\begin{array}{c}\text { Cell } \\
(e x: 22)\end{array}$ & Tree $\mathrm{N}$ & Tree Species & \multirow{2}{*}{\multicolumn{2}{|c|}{$\begin{array}{c}\begin{array}{c}\text { Cluster Heizht } \\
\text { (tep) (m) }\end{array} \\
9 \\
\end{array}$}} & $\begin{array}{l}\text { Clstr Height } \\
\text { (bottem) (m) }\end{array}$ \\
\hline & $40,45(92)$ & $E$ & $a 5$ & $7 \% 0$ & Eve & & & 9 \\
\hline & 20.37 (96) & $E$ & $a^{5}$ & $7 \%$ & Eve & \multicolumn{2}{|c|}{6} & 6 \\
\hline & $45,45 \quad(45)$ & D & 15 & 128 & Euc & \multicolumn{2}{|l|}{7} & 7 \\
\hline oroux & $50,60 \quad(55)$ & D & h6 & 134 & Eve & \multicolumn{2}{|l|}{6} & 6 \\
\hline$A 0480$ & $520 \quad(18)$ & $D$ & h6 & $T 34$ & Eve & \multicolumn{2}{|l|}{6} & 6 \\
\hline & $720,150(125)$ & $E$ & $c^{2}$ & 150 & $E$ & \multicolumn{2}{|l|}{4} & 6 \\
\hline & 90 , the $(105)$ & E & 62 & 150 & $E$ & \multicolumn{2}{|l|}{9} & 9 \\
\hline & $35,55(45)$ & E & $c^{2}$ & 150 & $E$ & \multicolumn{2}{|l|}{8} & 8 \\
\hline & $140,175(165)$ & $E$ & $c^{2}$ & 150 & E & \multicolumn{2}{|l|}{9} & 9 \\
\hline & $240,150(245)$ & E & $C 2$ & 150 & $E$ & \multicolumn{2}{|l|}{9} & 9 \\
\hline & $150, k 50(650)$ & $D$ & 12 & 163 & $E$ & \multicolumn{2}{|l|}{9} & 6 \\
\hline & $t 50,500(575)$ & D) & il & 763 & $e v$ & \multicolumn{2}{|l|}{8} & 8 \\
\hline & $00,900(250)$ & D & i2 & 763 & ex & \multicolumn{2}{|l|}{9} & 10 \\
\hline & now, noes $(16 \alpha)$ & $D$ & 14 & $T 70$ & $e x$ & \multicolumn{2}{|l|}{11} & 13 \\
\hline & $255.200(128)$ & D & +4 & $T 70$ & exe & \multicolumn{2}{|l|}{ I } & II \\
\hline & $380,80(425)$ & 1) & 24 & 770 & euc & \multicolumn{2}{|c|}{10} & 10 \\
\hline & \multicolumn{5}{|c|}{\begin{tabular}{|l|} 
Total in Clusters: \\
\end{tabular}} & \multicolumn{3}{|c|}{ Total II Trees: } \\
\hline & \multicolumn{5}{|l|}{ "Loners: H4 11} & & \multirow{4}{*}{\multicolumn{2}{|c|}{$\begin{array}{l}\text { Tally every butterfly of } \\
\text { each type that you see } \\
\text { throughout the site. }\end{array}$}} \\
\hline & \multicolumn{5}{|c|}{ \# Sunners: Xl 11} & & & \\
\hline & \multicolumn{5}{|c|}{\begin{tabular}{|l} 
\# Fliers: YHL (III \\
\# Grounders (live):
\end{tabular}} & \multirow{3}{*}{\multicolumn{3}{|c|}{\begin{tabular}{l|l} 
& throughout the site. \\
loners, sumers, fliers, etc)
\end{tabular}}} \\
\hline & \multicolumn{5}{|c|}{ \# Grounders (live): } & & & \\
\hline & \multicolumn{5}{|l|}{ Grand Total: } & & & \\
\hline
\end{tabular}

\begin{tabular}{|c|c|}
\hline Hating Monarehs: & \\
\hline Dead Menarchs: & \\
\hline Other Notes: & very light dizule $\triangle 7$ an \\
\hline
\end{tabular}




\begin{tabular}{|c|c|c|c|c|c|c|}
\hline $\begin{array}{l}\text { Wonarchs } \\
\text { Clustered }\end{array}$ & $\begin{array}{c}\text { Zone } \\
(e x: A)\end{array}$ & $\begin{array}{c}\text { Cell } \\
(\mathrm{ex}: \approx 2)\end{array}$ & Tree \# & Tree Species & $\begin{array}{c}\text { Cluster Height } \\
\text { (top) }(m)\end{array}$ & $\begin{array}{l}\text { Clstr Height } \\
\text { (bottom)(m) }\end{array}$ \\
\hline 450,600 (525) & $D$ & $d^{4} 4$ & 761 & Euc & 21 & 21 \\
\hline $75,200(188)$ & $D$ & $C^{4}$ & 769 & Evc & 21 & 21 \\
\hline $90,120(105)$ & $D$ & $e^{9}$ & 7108 & Cop & 12 & 12 \\
\hline $35,50(\pi)$ & D & eq & Tlor & Cyp & II & II \\
\hline $70,10 \quad(75)$ & D & eq & $7 / 08$ & Cyp & 12 & II \\
\hline $2000,2300(\mathrm{vr})$ & $D$ & b12 & $T 100$ & 6 & 12 & 11 \\
\hline $20,260(240)$ & 0 & 610 & 5100 & C & 12 & 12 \\
\hline $350,450(000)$ & D & 610 & $T 100$ & $C$ & 12 & 12 \\
\hline $760,650(675)$ & 0 & 610 & Tion & c & 14 & 14 \\
\hline $1040,1400(12 x)$ & $D$ & 210 & T100 & C & 21 & 20 \\
\hline $20,260(240)$ & $D$ & $\mathrm{ClO}$ & $T 100$ & $C$ & 20 & 20 \\
\hline $60,60(70)$ & $I$ & c1 & $T(a)$ & $C$ & b & 10 \\
\hline $110,150 \quad(130)$ & C & 18 & $T 81$ & EN & 8 & 8 \\
\hline $7980(75)$ & $C$ & 18 & 781 & Eur & 9 & 9 \\
\hline $200,220(225)$ & c & is & 781 & Euc & 12 & 10 \\
\hline 215250 (232) & $C$ & 9 & 781 & Eve & 8 & 8 \\
\hline $130,160(175)$ & $c$ & 19 & 781 & Euc & 9 & 8 \\
\hline$\frac{169 / 50(168)}{360.420(270)}$ & C & 4 & 72 & Eve & $n$ & 10 \\
\hline$\frac{350,420(310)}{240,220(2 \times 0)}$ & c & $h 8$ & 794 & Euc & 11 & 10 \\
\hline$\frac{240,520(230)}{270,320(295)}$ & C & hs & 194 & Ene & 9 & 9 \\
\hline$\frac{270,320(295)}{175,200(192)}$ & $C$ & h8 & T94 & Ene & 9 & 8 \\
\hline$\frac{175,200(192)}{80,80(50)}$ & $C$ & hs & 194 & are & 13 & 13 \\
\hline$\frac{80,80 \quad(00)}{220,220(220)}$ & I & $c 2$ & 799 & Eve & 22 & 22 \\
\hline $220,220(220)$ & $I$ & b3 & 799 & Eve & R & 12 \\
\hline & & & & & & \\
\hline & & & & & & \\
\hline & & & & & & \\
\hline & & & & & & \\
\hline & & & & & & \\
\hline & & & & & & \\
\hline & & & & & & \\
\hline & & & & & & \\
\hline & & & & & & \\
\hline
\end{tabular}

Please add any counts an this page to the total fally on the previous page. 


\section{Over-wintering Monarch Butterfly Project Monareh Count Data Sheet}

DATE $11 / 14 / 14$ SITE NAME Pismo

\#Observers 4 Observer name(s) Tyler, Brand; Sasmid, Jessica

Count Time Span 6:30 to Total Count Time (Min)

Cloud/Fog Cover $60 \%$ Precipitation (circle one) $\subset$ Tans) drizzle min

Temp ( $\left.{ }^{\circ} \mathrm{C}\right)+15 \cdot \tau^{\circ} \mathrm{LW}$ ind $(\mathrm{m} / \mathrm{s}) \_$ Wind (Besufort)

Wind direction

\begin{tabular}{|c|c|c|c|c|c|c|c|}
\hline $\begin{array}{l}\text { "Monarehs } \\
\text { Clestered }\end{array}$ & $\begin{array}{c}\text { Zone } \\
(\boldsymbol{e x :} \mathbf{A})\end{array}$ & $\underset{\left(\mathbf{c x :} \mathbf{a z}_{2}\right)}{\text { Cell }}$ & Tree \#I & Tree Species & \multicolumn{2}{|c|}{$\begin{array}{l}\text { Cluster Height } \\
\text { (top) (m) }\end{array}$} & $\begin{array}{l}\text { Clstr Height } \\
\text { (bottom) (m) }\end{array}$ \\
\hline $275 / 240$ & $I$ & h1 & 109 & 24 & \multicolumn{2}{|l|}{20} & 20 \\
\hline $100 / 125$ & $I$ & 1 & T109 & $\frac{6}{2}$ & \multicolumn{2}{|c|}{20} & 20 \\
\hline $150 / 180$ & D & $t 10$ & $T 100$ & $\bar{c}$ & \multicolumn{2}{|l|}{10} & 10 \\
\hline $1400 / 1250$ & D & c 9 & T100 & $c$ & \multicolumn{2}{|l|}{ II } & 10 \\
\hline $850 / 700$ & D & $c^{9}$ & $T 100$ & C & \multicolumn{2}{|l|}{13} & 12 \\
\hline $300 / 450$ & $D$ & $c^{\gamma}$ & T100 & $C$ & \multicolumn{2}{|l|}{11} & 11 \\
\hline $1760 / 1750$ & D & c9 & 1100 & $C$ & \multicolumn{2}{|l|}{18} & 17 \\
\hline $160 / 120$ & $I$ & b3 & 199 & E & \multicolumn{2}{|l|}{10} & 10 \\
\hline $120 / 90$ & $I$ & $\mathrm{Cl}$ & Ta9 & $\xi$ & \multicolumn{2}{|l|}{18} & 18 \\
\hline $120 / 135$ & $I$ & $c 2$ & Ta9 & $E$ & \multicolumn{2}{|c|}{16} & 16 \\
\hline $12 / 200$ & C & is & $T 91$ & $E$ & \multicolumn{2}{|l|}{ II } & 11 \\
\hline $320 / 350$ & C & is & $|9|$ & E & \multicolumn{2}{|l|}{9} & 9 \\
\hline $225 / 225$ & C & 18 & $T 91$ & $E$ & \multicolumn{2}{|c|}{12} & 12 \\
\hline$8 0 \longdiv { 7 5 }$ & C & is & 181 & $E$ & \multicolumn{2}{|c|}{8} & 8 \\
\hline $550 / 500$ & C & is & $\pi 81$ & $E$ & \multicolumn{2}{|c|}{12.} & II \\
\hline $125 / 100$ & $\bar{C}$ & 19 & $T \& 1$ & $E$ & \multicolumn{2}{|c|}{8} & 8 \\
\hline \multicolumn{5}{|c|}{ Total in Clusters: } & \multicolumn{3}{|c|}{ Total II Trees: } \\
\hline \multicolumn{5}{|l|}{ \# Loners: } & & \multirow{4}{*}{\multicolumn{2}{|c|}{$\begin{array}{l}\text { Tally every butterfly of } \\
\text { each type that you see } \\
\text { throughout the site. }\end{array}$}} \\
\hline \multicolumn{5}{|l|}{ W Sunners: } & & & \\
\hline \multicolumn{5}{|l|}{ W Fliers: } & & & \\
\hline \multicolumn{5}{|c|}{ W Grounders (live): } & & & \\
\hline \multicolumn{2}{|l|}{ Grand Total: } & fsum & of all chaste & ned butterflies pha & loners, sum & mers, flie & 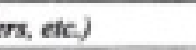 \\
\hline
\end{tabular}

\begin{tabular}{|l|l|}
\hline W Mating Monarchs: & \\
\hline W Dead Monarchst & \\
\hline Other Notes: & \\
\hline
\end{tabular}




\begin{tabular}{|c|c|c|c|c|c|c|}
\hline $\begin{array}{l}\text { Monarehs } \\
\text { Clustered }\end{array}$ & $\begin{array}{c}\text { Zone } \\
\text { (ex: A) }\end{array}$ & $\begin{array}{c}\text { Cell } \\
(\mathrm{cx}: \mathrm{a} 2)\end{array}$ & Tree H & Tree Species & $\begin{array}{c}\text { Cluster Height } \\
\text { (top) (m) }\end{array}$ & $\begin{array}{l}\text { Castr Height } \\
\text { (bottem) (m) }\end{array}$ \\
\hline $120 / 140$ & C & $h 7$ & 791 & $E$ & 4 & 9 \\
\hline $275 / 325$ & $c$ & $; 4$ & $T 7 b$ & C & 17 & 17 \\
\hline $350 / 400$ & C & 3 & $T 76$ & $C$ & 17 & 17 \\
\hline $250 / 275$ & $C$ & j4 & $T 76$ & $c$ & 14 & 14 \\
\hline $225 / 250$ & $D$ & 24 & T70 & $E$ & 8 & 8 \\
\hline $350 / 275$ & D & 64 & 725 & $E$ & 18 & 18 \\
\hline $400 / 350$ & D & C4 & 169 & $\varepsilon$ & 18 & 18 \\
\hline $150 / 130$ & $D$ & C 4 & 769 & $E$ & 15 & 15 \\
\hline $350 \% 475$ & $D$ & $d 4$ & 770 & E & 11 & 11 \\
\hline & & & & & & \\
\hline & & & & & & \\
\hline & & & & & & \\
\hline & & & & & & \\
\hline & & & & & & \\
\hline & & & & & & \\
\hline & & & & & & \\
\hline & & & & & & \\
\hline & & & & & & \\
\hline & & & & & & \\
\hline & & & & & & \\
\hline & & & & & & \\
\hline & & & & & & \\
\hline & & & & & & \\
\hline & & & & & & \\
\hline & & & & 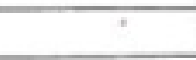 & & \\
\hline & & & & & & \\
\hline & & & & & & \\
\hline & & & & & & \\
\hline & & & & & & \\
\hline & & & & & & \\
\hline & & & & 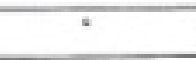 & & \\
\hline & & & & & & \\
\hline & & & & & & \\
\hline
\end{tabular}

Please add any counts on this page to the total tally an the previots page. 


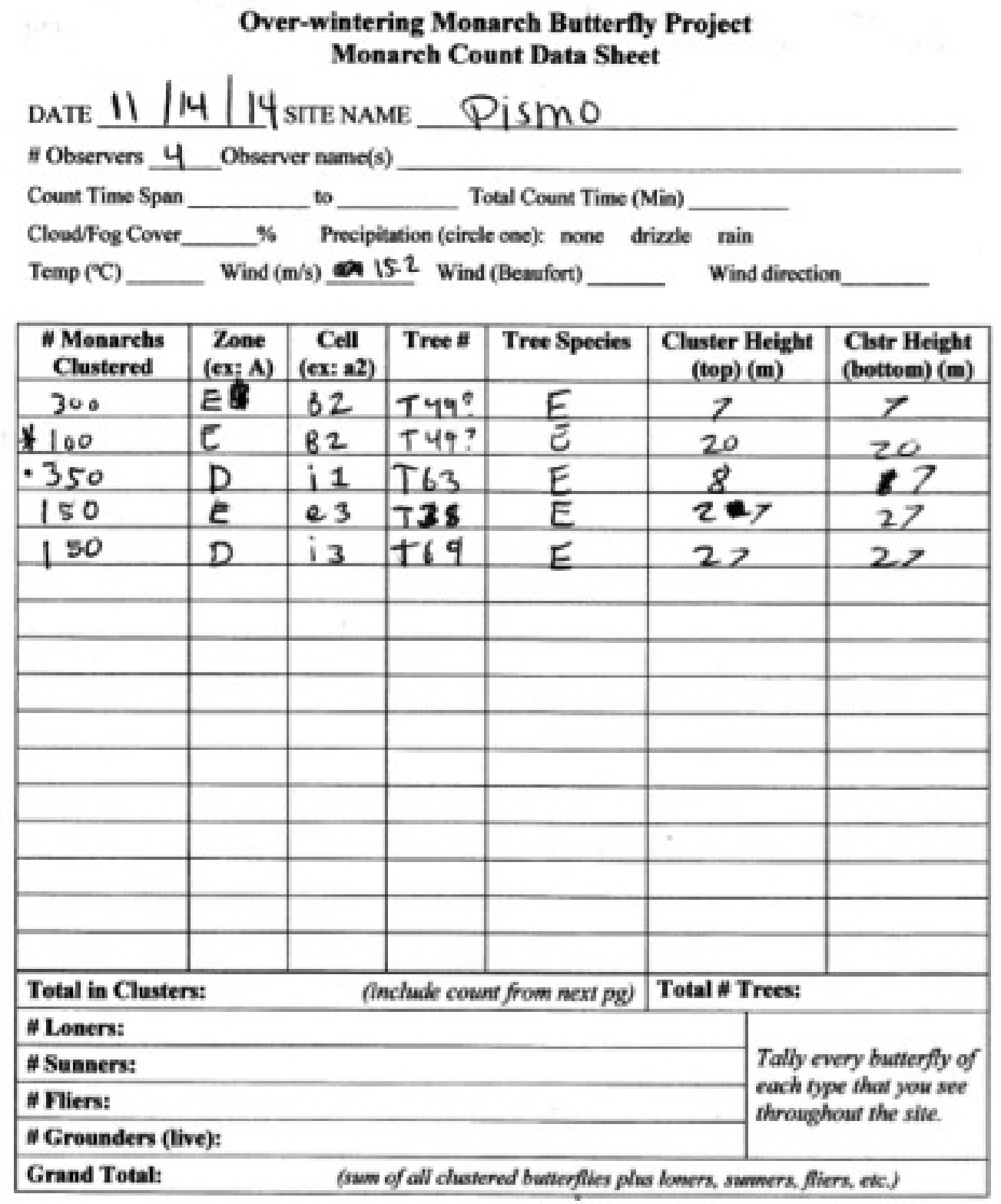

\begin{tabular}{|l|l|}
\hline \# Mating Monarchs: & \\
\hline \# Dead Monarchs: & \\
\hline Other Notes: & \\
\hline \\
* A 0554 - or anye. AO 254 - orany
\end{tabular}


Entent.

\section{Over-wintering Monarch Butterfly Project Monarch Count Data Sheet}

DATE $11 / 15 / 14$ SITENAME PISTO

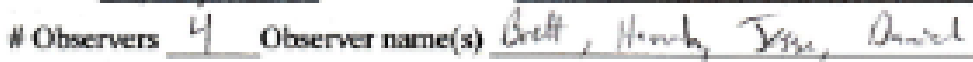

Count Time Span $6: 40 \%$ to 8 om Total Count Time (Min) 80

Cloud $/ \mathrm{Fog}_{\mathrm{C}} \mathrm{Cover} O \mathrm{O} \%$ Precipitation (circle one); (100) drizle rain

Temp (CC) 13.3 Wind (m/s) O.2 Wind (Beaufort)___ Wiad disertion LE

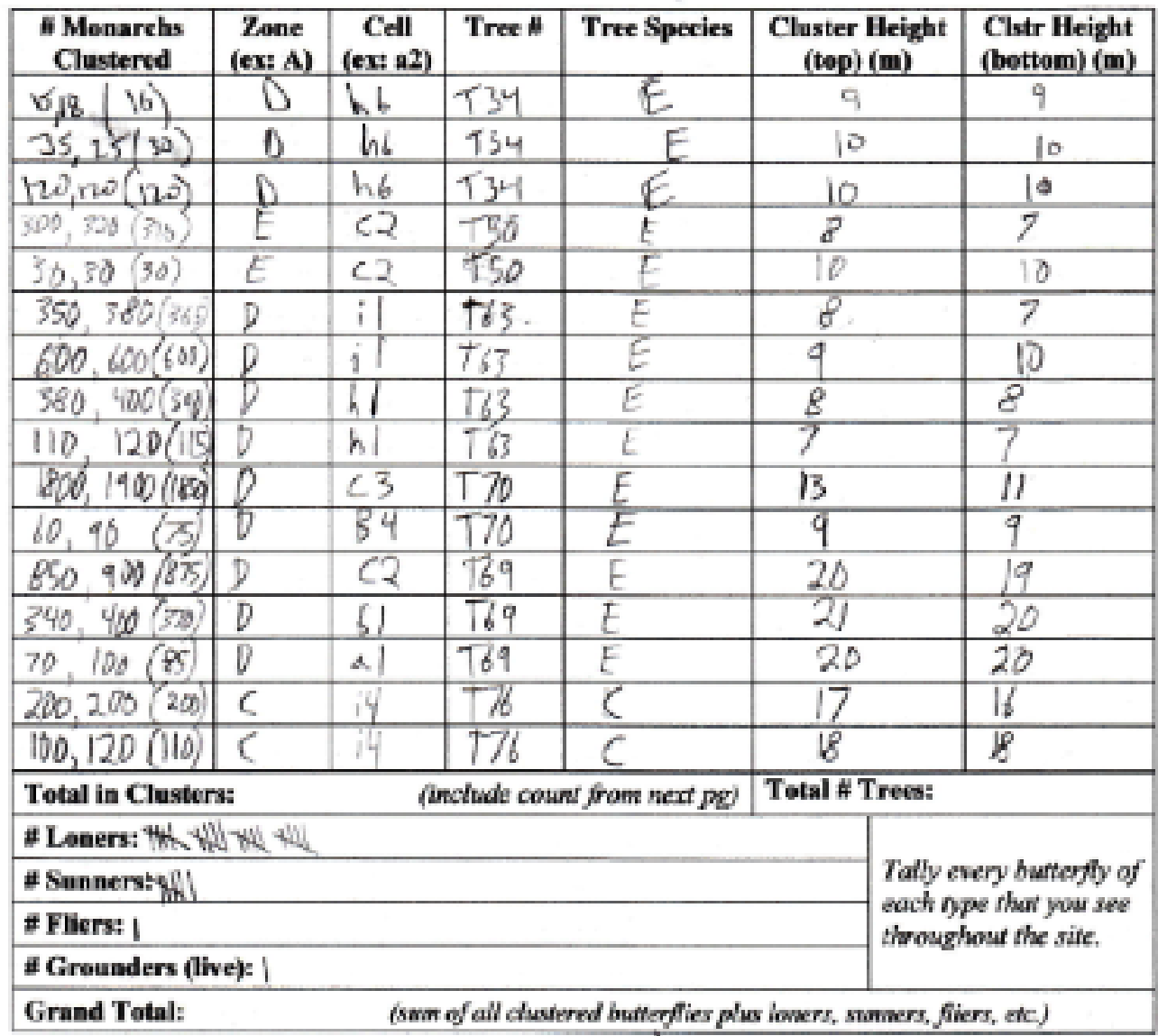

\begin{tabular}{|l|l|}
\hline \# Mating Monarchs: & \\
\hline Dead Menarehs: & 1 \\
\hline Other Notes: & \\
\hline
\end{tabular}




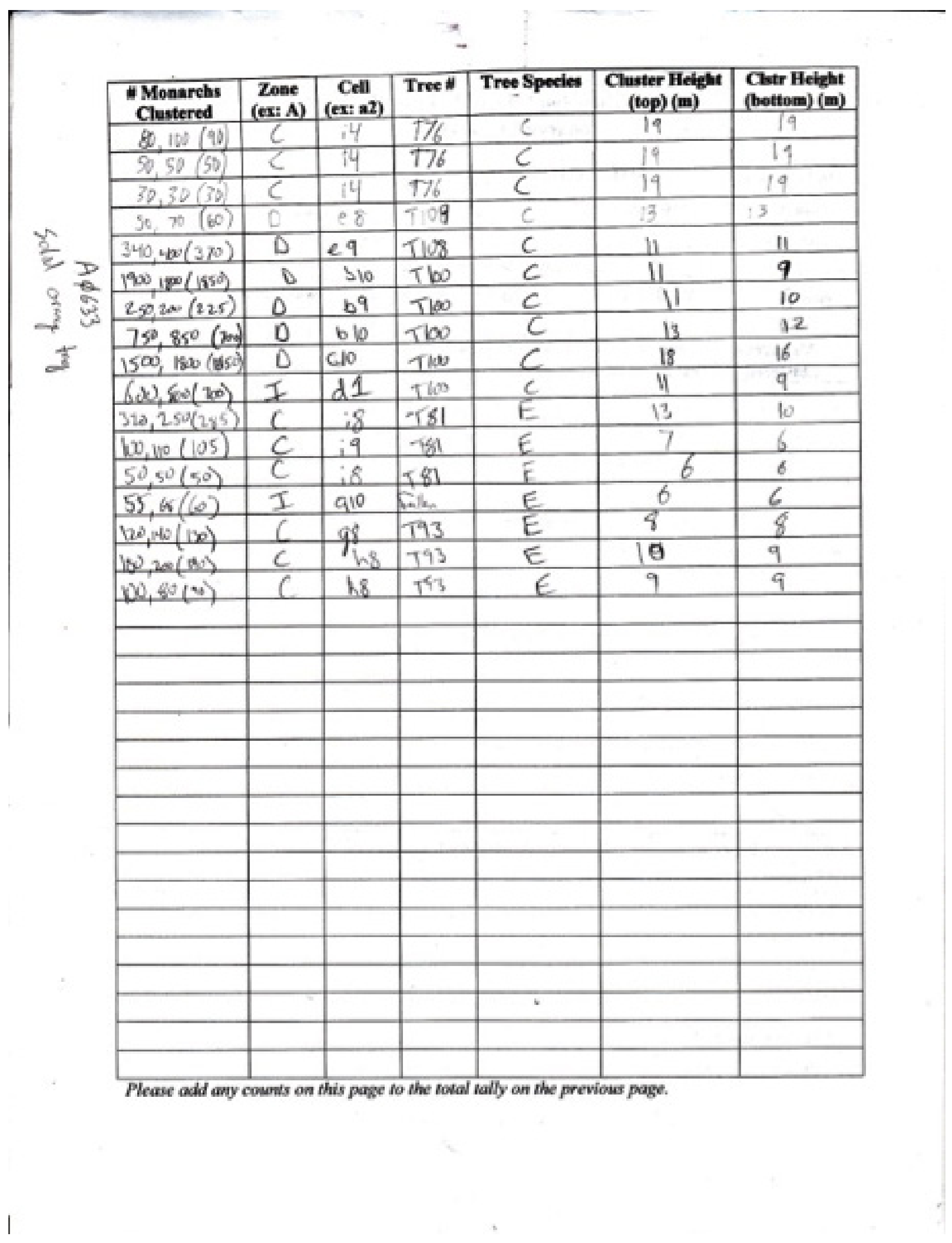


Envind

Over-wintering Monarch Butterfy Project Monarch Count Data Sheet

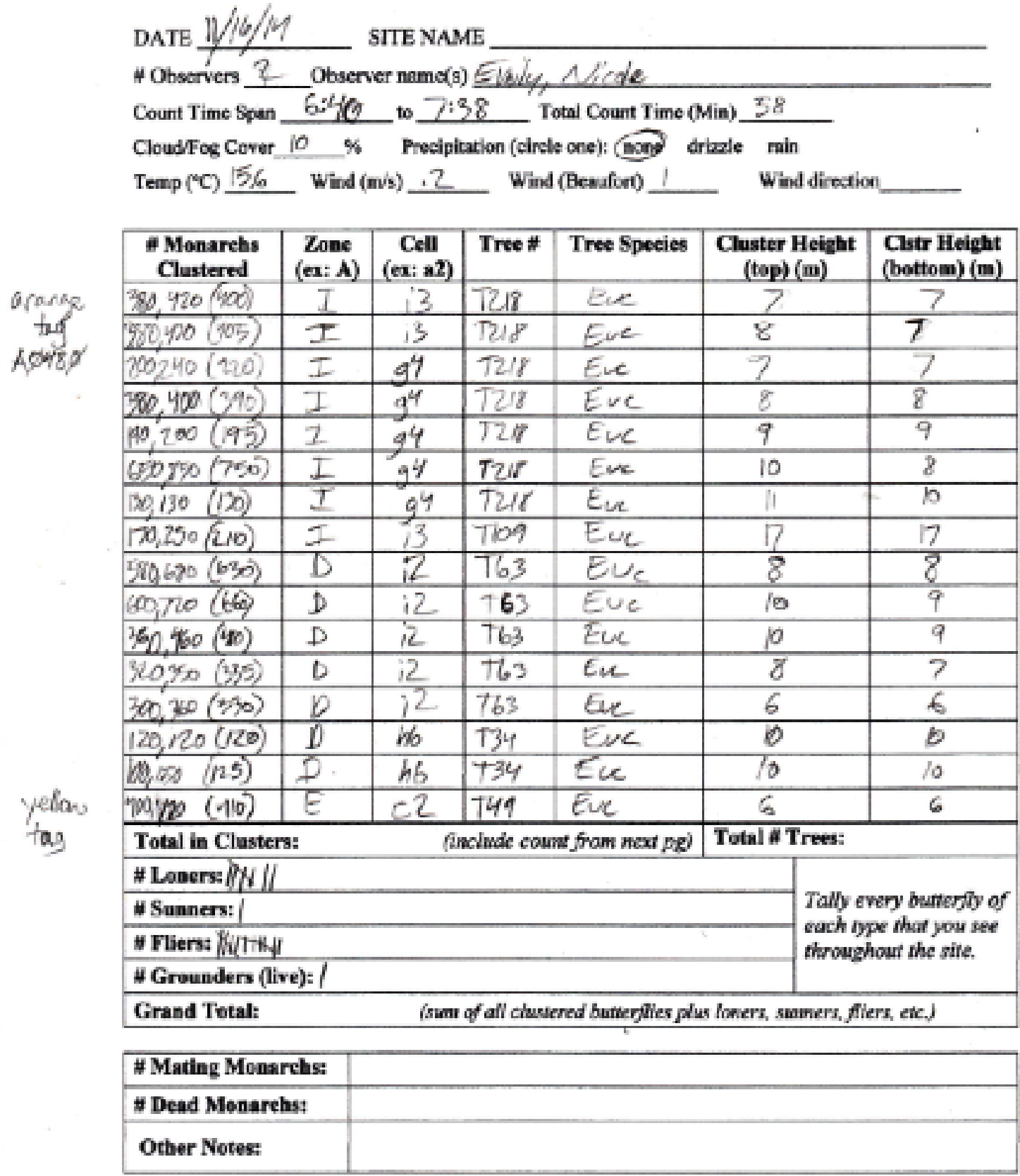




\section{Entid \\ Over-wintering Monarch Butterfly Project Monareh Count Data Sheet}

DATE $11-16-14$ sITE Name Prino

\# Observers 4 Observer name(s) Dnniel, lhanh

Count Time Span $6: 30 \quad$ to 7138 Total Count Time (Min)

Clond/Fog Cover_10 \% Precipitution (eircle one). none drizzle rain

Temp $(\mathrm{C}) \perp \mathrm{S} .6$ Wind $(\mathrm{m} / \mathrm{s})$ _. 0.2 Wind (Benufort) ___ Wind direction_W

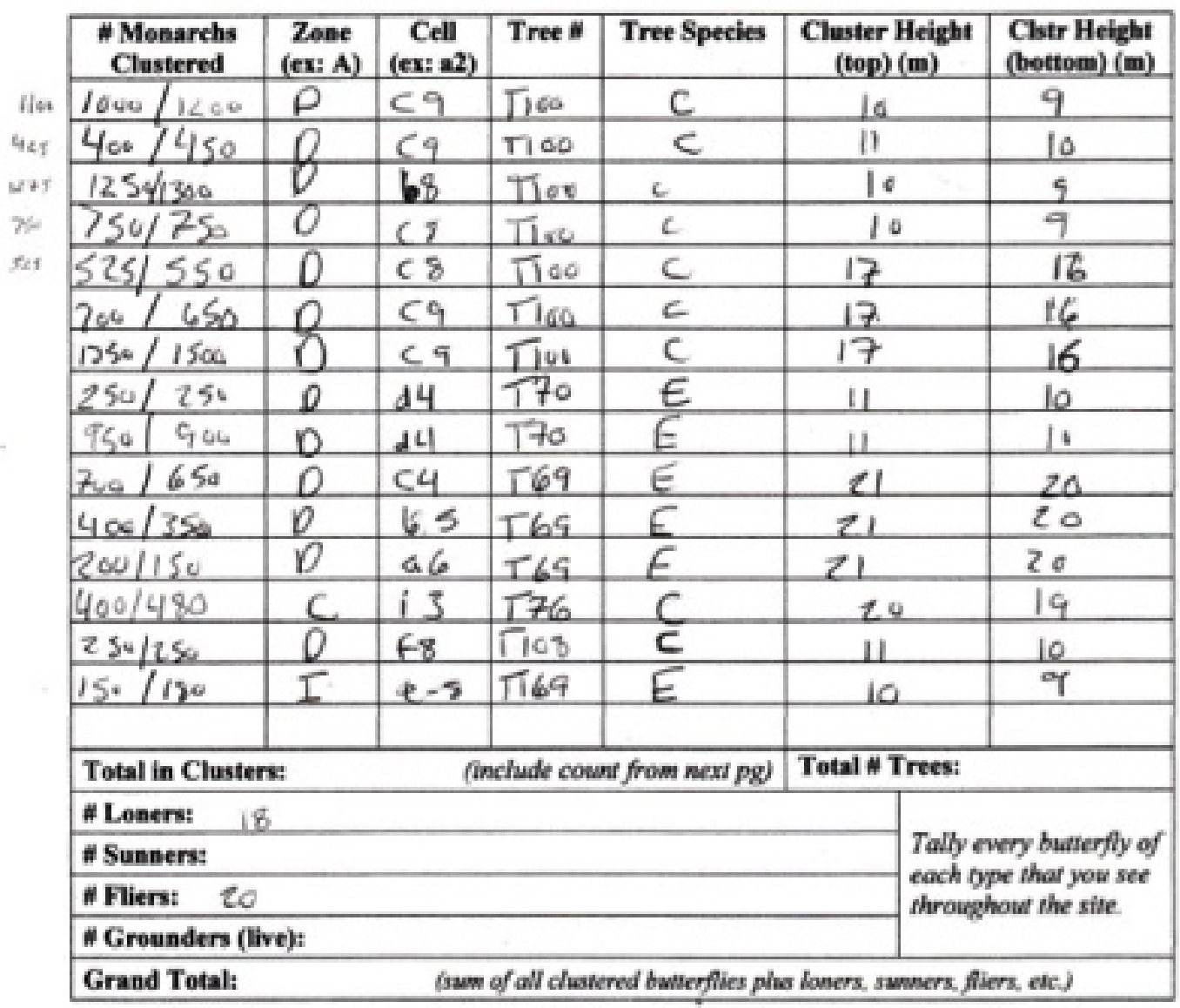

\begin{tabular}{|l|l|}
\hline "Mating Monarchs: & \\
\hline W Dead Monarchs: & \\
\hline Other Notes: & \\
\hline
\end{tabular}




\section{Over-wintering Monarch Butterfly Project Monareh Count Data Sheet}

DATE $11 \mathrm{NDV}$

SITE NAME Pismo

- Observers 3 Observer name(s) Daniel Tayler. Tyer

Count Time Span 00 to $7: 40$

Total Count Time (Min)

ClowdFog Cover_O \% Preciphation (circle one) (bone) drizzle rain Temp (C) $\frac{4.6 \%}{12.24 \mathrm{C}}$ Wind (m/s) 0.2 Wind (Beaufort) ___ Wind direction $\mathrm{N}$

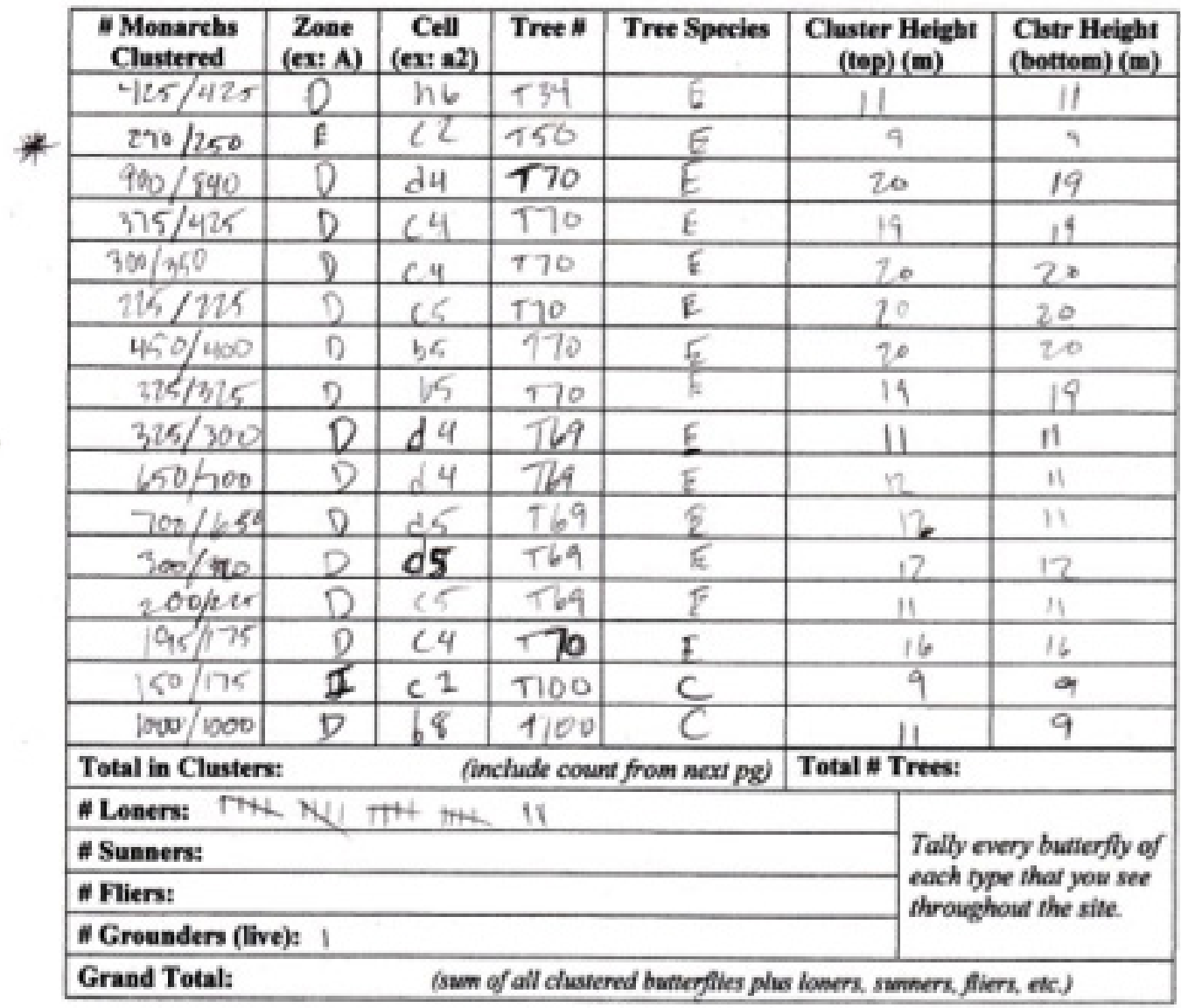

\begin{tabular}{|l|l|}
\hline N Mating Monarchs: & \\
\hline "Dead Monarehs: & \\
\hline Other Notes: & \\
\hline
\end{tabular}




\begin{tabular}{|c|c|c|c|c|c|c|}
\hline $\begin{array}{l}\text { Monarehs } \\
\text { Clustered }\end{array}$ & $\begin{array}{c}\text { Zone } \\
\text { (ex: A) }\end{array}$ & $\begin{array}{c}\text { Cell } \\
(\mathrm{ex:}: \mathbf{2}) \\
\end{array}$ & Tree \# & Tree Species & $\begin{array}{l}\text { Cluster Height } \\
\text { (top) (m) }\end{array}$ & $\begin{array}{l}\text { Cistr Height } \\
\text { (bottom) (m) }\end{array}$ \\
\hline $350 / 400$ & $D$ & 88 & 1100 & $C$ & 11 & 11 \\
\hline $300 / 3$ & $D$ & ct & $T 100$ & $C$ & 12 & 10 \\
\hline $400 / 420$ & $D$ & $d g$ & 7100 & $C$ & 17 & 16 \\
\hline $1306 / 130$ & D & $d^{9}$ & $\pi 100$ & C & 18 & 17 \\
\hline 1350 / tho & $D$ & $d^{9}$ & Two & C & 15 & 17 \\
\hline$3 5 0 \longdiv { 4 0 0 }$ & C & $i 4$ & 776 & C & 16 & 16 \\
\hline $300 / 30$ & C & i4 & $T 76$ & $C$ & 17 & 17 \\
\hline $30 / 210$ & C & 18 & 181 & $E$ & 12 & 12 \\
\hline $250 / 280$ & $c$ & h8 & 194 & $E$ & 12 & 12 \\
\hline $320 / 260$ & $D$ & i1 & 763 & E & 8 & 8 \\
\hline $150 / 150$ & $D$ & $h L$ & $T 63$ & $F$ & 10 & 10 \\
\hline 2001175 & D & $i 2$ & $\pi 3$ & 6 & 11 & 1) \\
\hline $240 / 256$ & $D$ & 94 & 138 & E & 24 & 24 \\
\hline & & & & & & \\
\hline & & & & & & \\
\hline & & & & & & \\
\hline & & & & & & \\
\hline & & & & & & \\
\hline & & & & & & \\
\hline & & & & & & \\
\hline & & & & & & \\
\hline & & & & & & \\
\hline & & & & & & \\
\hline & & & & & & \\
\hline & & & & & & \\
\hline & & & & & & \\
\hline & & & & & & \\
\hline & & & & & & \\
\hline & & & & & & \\
\hline & & & & & & \\
\hline & & & & $\checkmark$ & & \\
\hline & & & & & & \\
\hline & & & & & & \\
\hline
\end{tabular}

Please add ary cownts an this page to the total tally an the prrvious page. 


\section{Over-wintering Monarch Butterfly Project Monareh Count Data Sheet}

DATE $\Perp / 18 / 14$ SITE NAME $\frac{P \text { ismo }}{0}$

" Observers ___ Observer name(s) Brett, Galy, Tesive, Nive

CloudFog Cover, $30 \%$ Precipitation (circle one) Ecos drizzle rain

Temp (C) $\mathrm{So}^{\circ \mathrm{F}}$ Wind (m/s) $\mathrm{O}$ Wind (Beaufort) $\mathrm{O}$ Wind direction

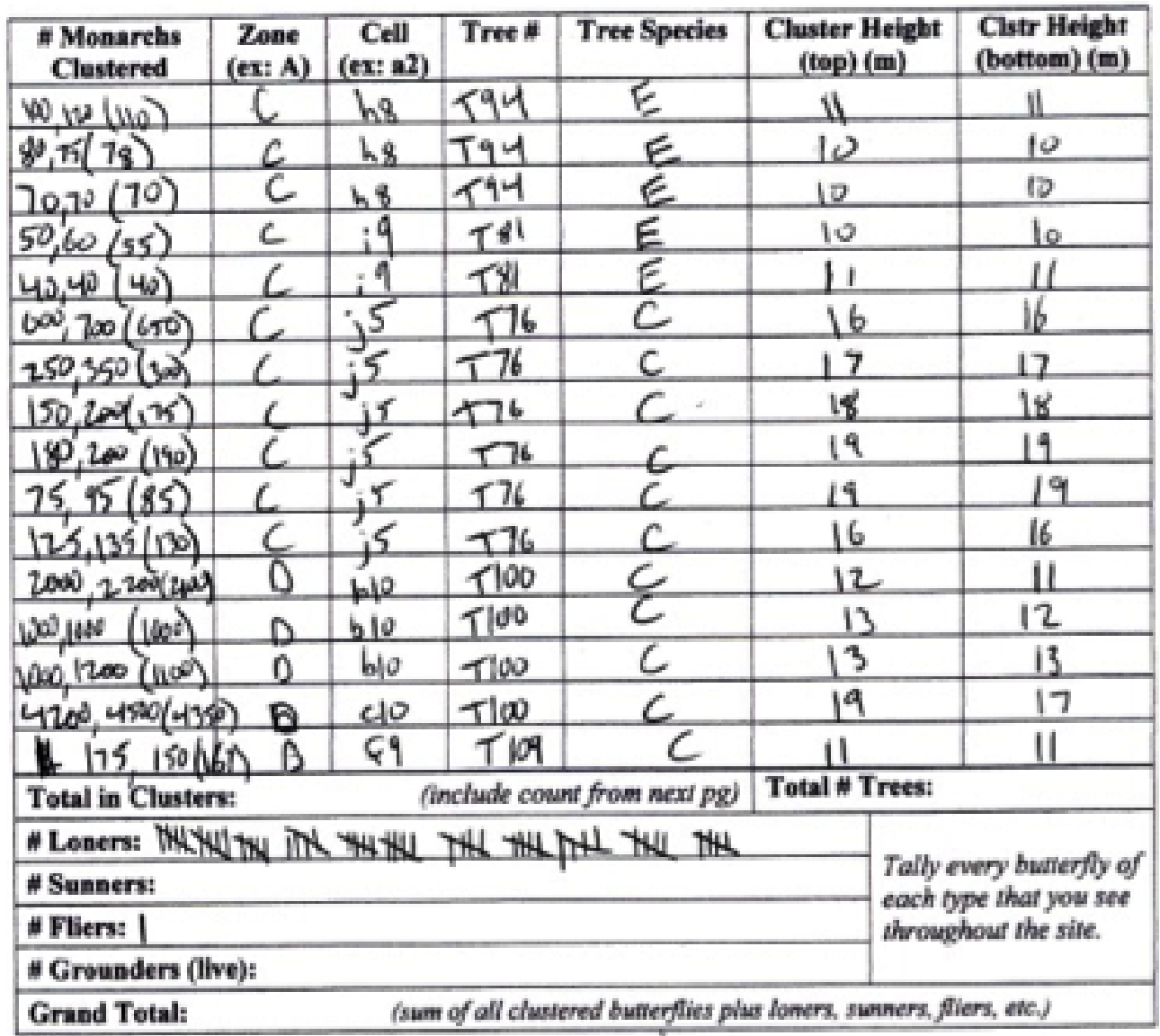

\begin{tabular}{|l|l|}
\hline W Mating Monarchs: & \\
\hline \# Dead Monarehs: & \\
\hline Other Notes: & \\
\hline
\end{tabular}




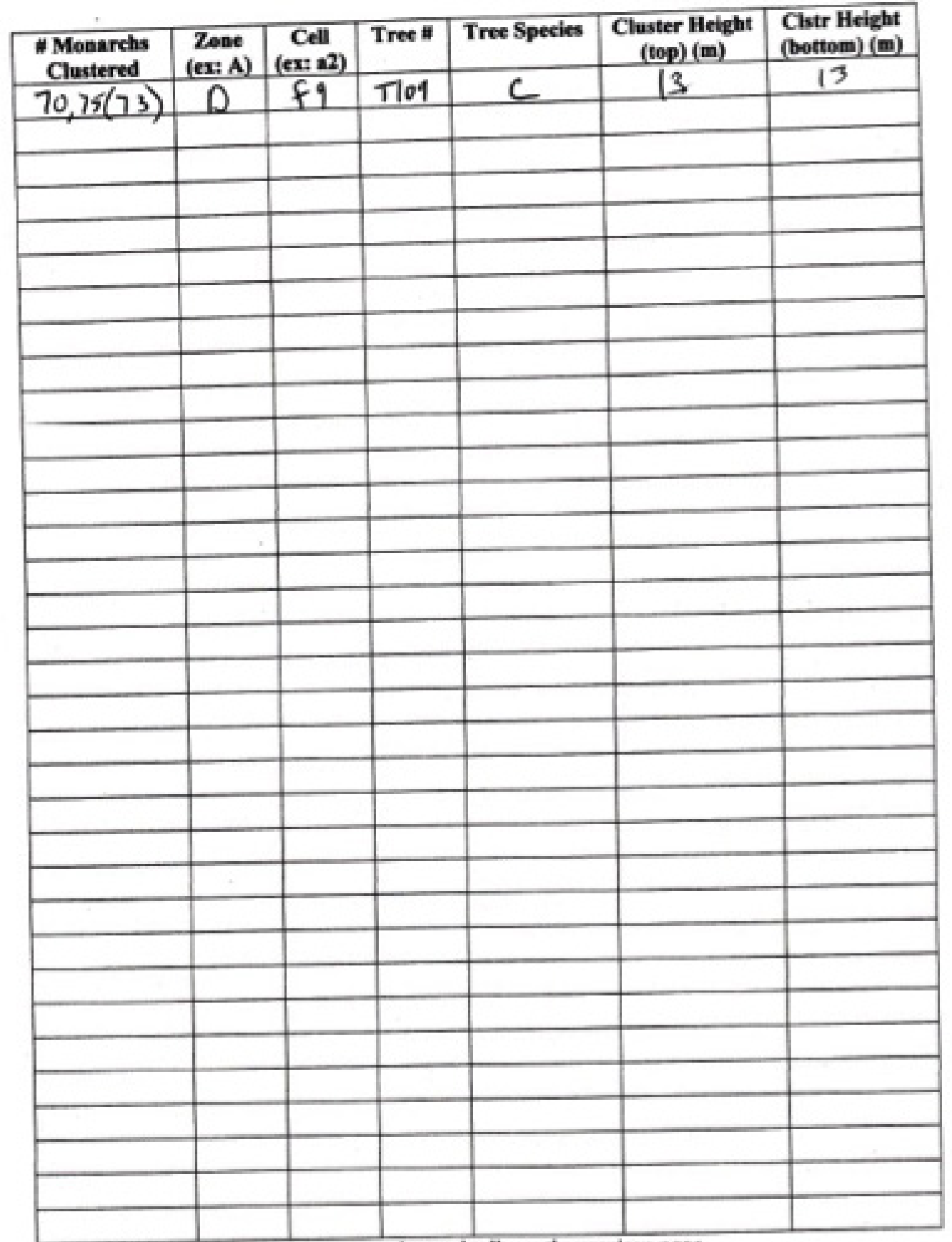

Please add any coumts on this page to the toial tally on the previous page. 


\section{Over-wintering Monarch Butterfly Project Monarch Count Data Sheet}

DATE $1 / / / 8 / / 4$ SITE NAME Piomo

\# Observers Z Observer name(s) Nicole, Enity

Count Time Span $6: 42$ to $7: 33$ Total Count Time (Min) 51

Cloud/Fog Cover_ 30 \% Precipitatice (eircle one) (none) drizzle nim

Temp (C) $\frac{}{5 O^{*}}$ Wind $(\mathrm{m} / \mathrm{s}) \mathrm{O}$ Wind (Beaufort) $\mathrm{O}$ Wind direction

\begin{tabular}{|c|c|c|c|c|c|c|c|}
\hline $\begin{array}{l}\text { Monarehs } \\
\text { Clustered }\end{array}$ & $\begin{array}{c}\text { Zone } \\
(\mathbf{e x :}: \mathbf{A})\end{array}$ & $\begin{array}{c}\text { Cell } \\
(e x: \geq 2)\end{array}$ & Tree $\|$ & Tree Species & \multirow{2}{*}{\multicolumn{2}{|c|}{$\begin{array}{c}\begin{array}{c}\text { Claster Height } \\
\text { (top) (m) }\end{array} \\
12\end{array}$}} & $\begin{array}{l}\text { Clstr Height } \\
\text { (bottom) (m) }\end{array}$ \\
\hline $170,130(130)$ & $I$ & $g^{4}$ & 7218 & Eve & & & 9 \\
\hline $25,30(28)$ & E & $a^{6}$ & 739 & Eve & \multicolumn{2}{|l|}{$\frac{9}{9}$} & 9 \\
\hline $10,120(115)$ & $D$ & 24 & 734 & Eur & \multicolumn{2}{|l|}{9} & 9 \\
\hline $60,86(70)$ & D & $e 4$ & 734 & Eve & \multicolumn{2}{|l|}{$p$} & $b$ \\
\hline $6285(75)$ & D & of & 734 & EUL & \multicolumn{2}{|l|}{$D$} & $b$ \\
\hline $17,180(175)$ & D & ey & 134 & Eve & \multicolumn{2}{|l|}{11} & 10 \\
\hline $25,70(28)$ & D & $e^{4}$ & 734 & Eve & \multicolumn{2}{|l|}{$n$} & 12 \\
\hline $30,400 \quad(390)$ & D & $i 2$ & 763 & Eve & \multicolumn{2}{|l|}{7} & 7 \\
\hline $10,160(150)$ & D & 12 & 763 & Ex & \multicolumn{2}{|l|}{9} & 9 \\
\hline $120560(990)$ & $D$ & 12 & Tb3 & en & \multicolumn{2}{|l|}{ II } & 10 \\
\hline $00,260(220)$ & $E$ & 22 & Ty9 & Euc & \multicolumn{2}{|l|}{7} & 7 \\
\hline $14780(440)$ & D & $d 4$ & 170 & Euc & \multicolumn{2}{|l|}{8} & 8 \\
\hline $600,1700(1650)$ & D & $d 4$ & $T 70$ & Euc & \multicolumn{2}{|l|}{10} & 9 \\
\hline $230,300(265)$ & b & $d^{4}$ & 170 & Euc & \multicolumn{2}{|l|}{8} & 8 \\
\hline $154,155(153)$ & D & $d^{3}$ & T69 & Eu & \multicolumn{2}{|l|}{12} & 12 \\
\hline $550,600(5,75)$ & 0 & $d 3$ & 169 & Ex & \multicolumn{2}{|l|}{20} & 19 \\
\hline \multirow{2}{*}{\multicolumn{5}{|c|}{$\begin{array}{l}\text { Total in Clusters: } \\
\text { " Lonerss } \mathrm{KW} \text { । }\end{array}$}} & \multicolumn{3}{|c|}{ Total | Trees: } \\
\hline & \multicolumn{4}{|c|}{$\begin{array}{l}\text { \# Loners: NXI } \\
\text { W Sunners: }\end{array}$} & & \multirow{4}{*}{\multicolumn{2}{|c|}{$\begin{array}{l}\text { Tally every buatterfly of } \\
\text { each type that you see } \\
\text { throughout the site. }\end{array}$}} \\
\hline W Sunaers: & & & & & & & \\
\hline Wliers: & & & & & & & \\
\hline \multicolumn{5}{|c|}{ \# Grounders (live): / } & \multirow{2}{*}{\multicolumn{3}{|c|}{ uoners, fliers, ete) }} \\
\hline Grand Total: & & & & & & & \\
\hline
\end{tabular}

\begin{tabular}{|l|l|}
\hline W Mating Monarchs: & \\
\hline " Dead Monarchs: & I \\
\hline Other Notes: & \\
\hline
\end{tabular}




\begin{tabular}{|c|c|c|c|c|c|c|}
\hline $\begin{array}{l}\text { Monarehs } \\
\text { Clustered }\end{array}$ & $\begin{array}{c}\text { Zone } \\
\text { (ex: A) }\end{array}$ & $\begin{array}{c}\text { Cell } \\
(\mathrm{ex} ; \mathrm{a2})\end{array}$ & Tree & Tree Species & $\begin{array}{l}\text { Cluster Height } \\
\text { (top) (m) }\end{array}$ & $\begin{array}{l}\text { Clstr Height } \\
\text { (bottom) (m) }\end{array}$ \\
\hline $100,40 \quad(405)$ & $D$ & $\sqrt{3}$ & 769 & Euc & 19 & 18 \\
\hline $320,360(310)$ & D & $2 \sqrt{3}$ & 769 & Enc & 20 & 20 \\
\hline $160,700(180)$ & D & $d^{3}$ & 769 & Eve & 20 & 20 \\
\hline $20,300(265)$ & $D$ & $d^{3}$ & Tbq & Euc & 19 & 19 \\
\hline & & & & & & \\
\hline & & & & & & \\
\hline & & & & & & \\
\hline & & & & & & \\
\hline & & & & & & \\
\hline & & & & & & \\
\hline & & & & & & \\
\hline & & & & & & \\
\hline & & & & & & \\
\hline & & & & & & \\
\hline & & & & & & \\
\hline & & & & & & \\
\hline & & & & & & \\
\hline & & & & & & \\
\hline & & & & & & \\
\hline & & & & & & \\
\hline & & & & & & \\
\hline & & & & & & \\
\hline & & & & & & \\
\hline & & & & & & \\
\hline & & & & & & \\
\hline & & & & & & \\
\hline & & & & & & \\
\hline & & & & & & \\
\hline & & & & & & \\
\hline & & & & & & \\
\hline & & & & & & \\
\hline & & & & & & \\
\hline & & & & & & \\
\hline & & & & & & \\
\hline
\end{tabular}




\section{Over-wintering Monarch Butterfly Projeet Monareh Count Data Sheet}

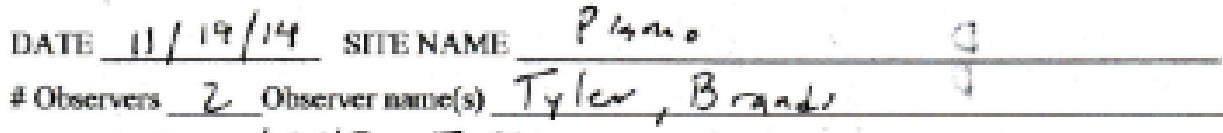
Count Time Spen $6: 45$ to $7: 32$ Total Coust Timx (Min) Cloudfog Cover $95 \%$ Precipitation (eirele anc) actione drizak rain Temp ("C) 12.7 Wind (m/s) 0.00 Wind (Besufart) Wind direction

\begin{tabular}{|c|c|c|c|c|c|c|}
\hline $\begin{array}{l}\text { HMaschs } \\
\text { Clustered }\end{array}$ & $\begin{array}{c}\text { Zone } \\
\text { (ex: } \boldsymbol{A})\end{array}$ & \begin{tabular}{|c|}
$\begin{array}{c}\text { Cell } \\
\text { (ex: a2) }\end{array}$ \\
\end{tabular} & Tree A & Tree Species & \multirow{2}{*}{$\begin{array}{c}\begin{array}{c}\text { Cluster Height } \\
\text { (top) (m) }\end{array} \\
11 \\
\end{array}$} & $\begin{array}{l}\text { Clstr Height } \\
\text { (bottom) (m) }\end{array}$ \\
\hline $250 / 230$ & C & h9 & $T 94$ & $E$ & & 11 \\
\hline $200 / 190$ & $c$ & 18 & $T \times 1$ & É & /1 & /1 \\
\hline $475 / 400$ & $D$ & $c / 0$ & $T 100$ & c & 10 & 10 \\
\hline $2000 / 1750$ & D & by & 7200 & $C$ & 11 & 10 \\
\hline $1450 / 1300$ & D & 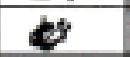 & T100 & $c$ & 11 & 10 \\
\hline $4000 / 3800$ & $D$ & $d \theta$ & $T / 00$ & $<$ & 12 & 16 \\
\hline $600 / 675$ & C & $i 6$ & 776 & C & *⿻17 & 16 \\
\hline $405 / 450$ & D & 65 & T69 & E & 18 & 18 \\
\hline $300 / 350$ & $D$ & $d y=4$ & T69 & E & 17 & 17 \\
\hline $125 / 100$ & D & $84 c 4$ & ra & $E$ & 5 & 15 \\
\hline $260 / 225$ & D & $c 4$ & Tho & E & 9 & 9 \\
\hline $100 / 1050$ & $D$ & $d 4$ & $T 7 D$ & $E$ & i) & 10 \\
\hline $42 \pi / 425$ & D & $d y$ & T70 & $E$ & 9 & 8 \\
\hline $560 / 200$ & D & iz & $T_{63}$ & $\sqrt{2}$ & 12 & 11 \\
\hline $100 / 200$ & 7 & 11 & 763 & $E$ & 10 & 10 \\
\hline $306 / 2 \mathrm{x}$ & D & it & 763 & $E$ & 8 & 8 \\
\hline \multicolumn{2}{|c|}{ Total in Clusiers: } & \multirow{2}{*}{\multicolumn{3}{|c|}{ (inchude conin from next pD) }} & \multicolumn{2}{|l|}{ Total \# Trees: } \\
\hline \multicolumn{3}{|l|}{ I Loners: } & & & \multirow{4}{*}{\multicolumn{2}{|c|}{$\begin{array}{l}\text { Tally avery butterfity of } \\
\text { each type that you see } \\
\text { throughout the sile. }\end{array}$}} \\
\hline \multicolumn{5}{|l|}{ \# Sunners: } & & \\
\hline \multicolumn{5}{|l|}{ \# Fliers: ! } & & \\
\hline \multicolumn{5}{|c|}{ \# Grounders (live): } & & \\
\hline \multicolumn{2}{|l|}{ Grund Total: } & \multicolumn{5}{|c|}{ 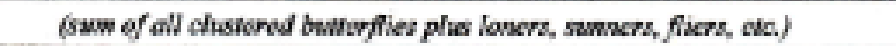 } \\
\hline
\end{tabular}

\begin{tabular}{|l|l|}
\hline \# Mating Manarchs: & \\
\hline \# Dead Monarchs: & \\
\hline Other Notes: & \\
\hline
\end{tabular}




\begin{tabular}{|c|c|c|c|c|c|c|}
\hline $\begin{array}{l}\text { Menarchs } \\
\text { Clustered }\end{array}$ & $\begin{array}{c}\text { Zone } \\
\text { (ex: A) }\end{array}$ & $\begin{array}{c}\text { Cell } \\
(e x: \mathbf{a} 2)\end{array}$ & Tree \# & Tree Species & $\begin{array}{c}\text { Cluster Height } \\
\text { (top) }(\mathbf{m})\end{array}$ & $\begin{array}{l}\text { Cistr Height } \\
\text { (bottom) (m) }\end{array}$ \\
\hline $275 / 25$ & e & $c^{2}$ & $T^{50}$ & $E$ & 8 & 8 \\
\hline $425 / 315$ & D & $h^{6}$ & 134 & E & 9 & 9 \\
\hline $200 / 150$ & D & $h_{3}$ & $T 38$ & $E$ & 23 & 25 \\
\hline $125 / 150$ & 0 & 93 & T69 & $\varepsilon$ & 23 & 23 \\
\hline \multirow{2}{*}{\multicolumn{7}{|c|}{$\frac{1237150}{400 / 350}$}} \\
\hline & & & & & & \\
\hline & & & & & & \\
\hline & & & & & & \\
\hline & & & & & & \\
\hline & & & & & & \\
\hline & & & & & & \\
\hline & & & & & . & \\
\hline & & & & & & \\
\hline & 81 & & & . & & \\
\hline & & & & & & \\
\hline & & & & & & \\
\hline & & & & & & \\
\hline & & & & & & \\
\hline & & & & & & \\
\hline & & & & & & \\
\hline$\cdot$ & & & & & & \\
\hline & & & & 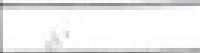 & & \\
\hline & & & & & & \\
\hline & & & & & & \\
\hline & & & & & & \\
\hline & & & & & & \\
\hline & & & & & & \\
\hline & & & & & & \\
\hline & & & & & & \\
\hline & & & & & & \\
\hline & & & & & & \\
\hline & & & & & & \\
\hline & & & & & & \\
\hline & & & & & & \\
\hline
\end{tabular}

Please add any counts on this page to the tolal tally ow the previons page. 


\section{Over-wintering Monarch Butterfly Project Monareh Count Data Sheet}

Date $\frac{1 / 20 / 44}{1}$ sIte name Piono

\# Observers 3 Observer name(s) Bntt, Emily, Nicole

Count Time Span 6.45or to 7:50 Total Count Time (Min) 65

Cloud/Fog Cover $100 \%$ Precipitation (circle one):

Temp (C) $\frac{}{6 \sigma F}$ Wind (m/s) 0.2 Wind (Beaufort) ___ Wind direction $N$

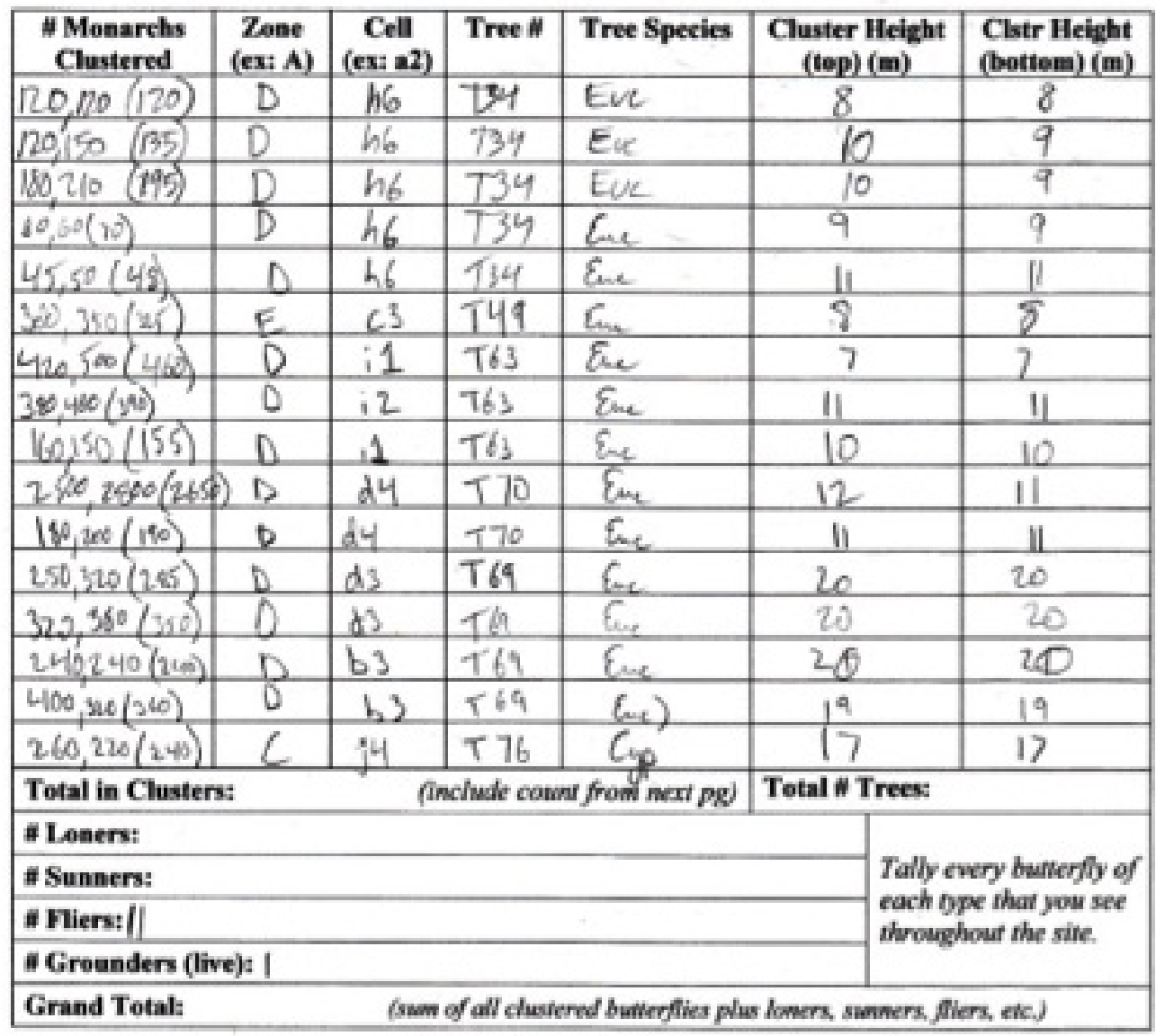

\begin{tabular}{|l|l|}
\hline I Mating Monarchs: & \\
\hline "Dead Monarchs: & \\
\hline Other Notes: & Orange toy \\
\hline
\end{tabular}




\begin{tabular}{|c|c|c|c|c|c|c|}
\hline $\begin{array}{l}\text { Monarehs } \\
\text { Clustered }\end{array}$ & $\begin{array}{c}\text { Zone } \\
\text { (ex: A) }\end{array}$ & $\begin{array}{c}\text { Cell } \\
(\mathbf{e x :} 22)\end{array}$ & Tree "ll & Tree Species & $\begin{array}{l}\text { Cluster Height } \\
\text { (top) (m) }\end{array}$ & $\begin{array}{l}\text { Ckstr Height } \\
\text { (bettom) (m) }\end{array}$ \\
\hline $160,140(150)$ & $C$ & $j 4$ & $T 76$ & $\operatorname{lop}$ & 17 & 17 \\
\hline $80,120(100)^{\prime}$ & $C$ & 4 & $T 76$ & $\mathrm{cop}$ & 18 & 18 \\
\hline $100,120(110)$ & $D$ & es & $T 108$ & ${ }_{c p}$ & 12 & 12 \\
\hline $240,380(200)$ & D & 48 & Ties & $C_{\infty}$ & 10 & 10 \\
\hline $60,75(68)$ & $I$ & c1 & $T 100$ & $d_{\text {cop }}$ & 10 & 10 \\
\hline $1500,1800(1050)$ & D & 610 & 1160 & $G_{p}$ & 10 & 9 \\
\hline $650,690(605)$ & D & 610 & $T 100$ & $G_{p}$ & 11 & 10 \\
\hline $1200,1400(1200)$ & $D$ & 69 & $T 100$ & a & 12 & "I \\
\hline $5000,550(5250)^{\prime}$ & $I$ & c1 & 1100 & Cyp & 20 & 19 \\
\hline $420,28 \times(400)$ & D & $e 7$ & T104 & E.c. & 20 & 20 \\
\hline $50,40(45)$ & $C$ & i9 & T\&1 & Ene & 8 & 8 \\
\hline $120,90(105)$ & $c$ & 1 & 781 & he & 10 & 10 \\
\hline $100,120(110)$ & $c$ & 18 & $T \& 1$ & $\sum$ & 12 & 12 \\
\hline $320,312(-345)$ & C & h8 & T94 & $\varepsilon_{2}$ & $\Perp$ & 10 \\
\hline & & & & & & \\
\hline $26 t_{2}$ & $C$ & h9 & $T 42$ & Gif. & 13 & 11 \\
\hline & & & & & & \\
\hline & & & & & & \\
\hline & & & & & & \\
\hline 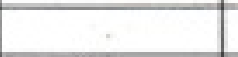 & & & & & & \\
\hline & & & & & & \\
\hline & & & & & $=$ & \\
\hline & & & & & & \\
\hline & & & & & & \\
\hline & & & & & & \\
\hline & & & & & & \\
\hline & & & & & & \\
\hline & & & & & & \\
\hline & & & & & & \\
\hline & & & & & & \\
\hline & & & & & & \\
\hline & & & & & & \\
\hline
\end{tabular}

Please add any counts on this page to the total tally an the previous page. 


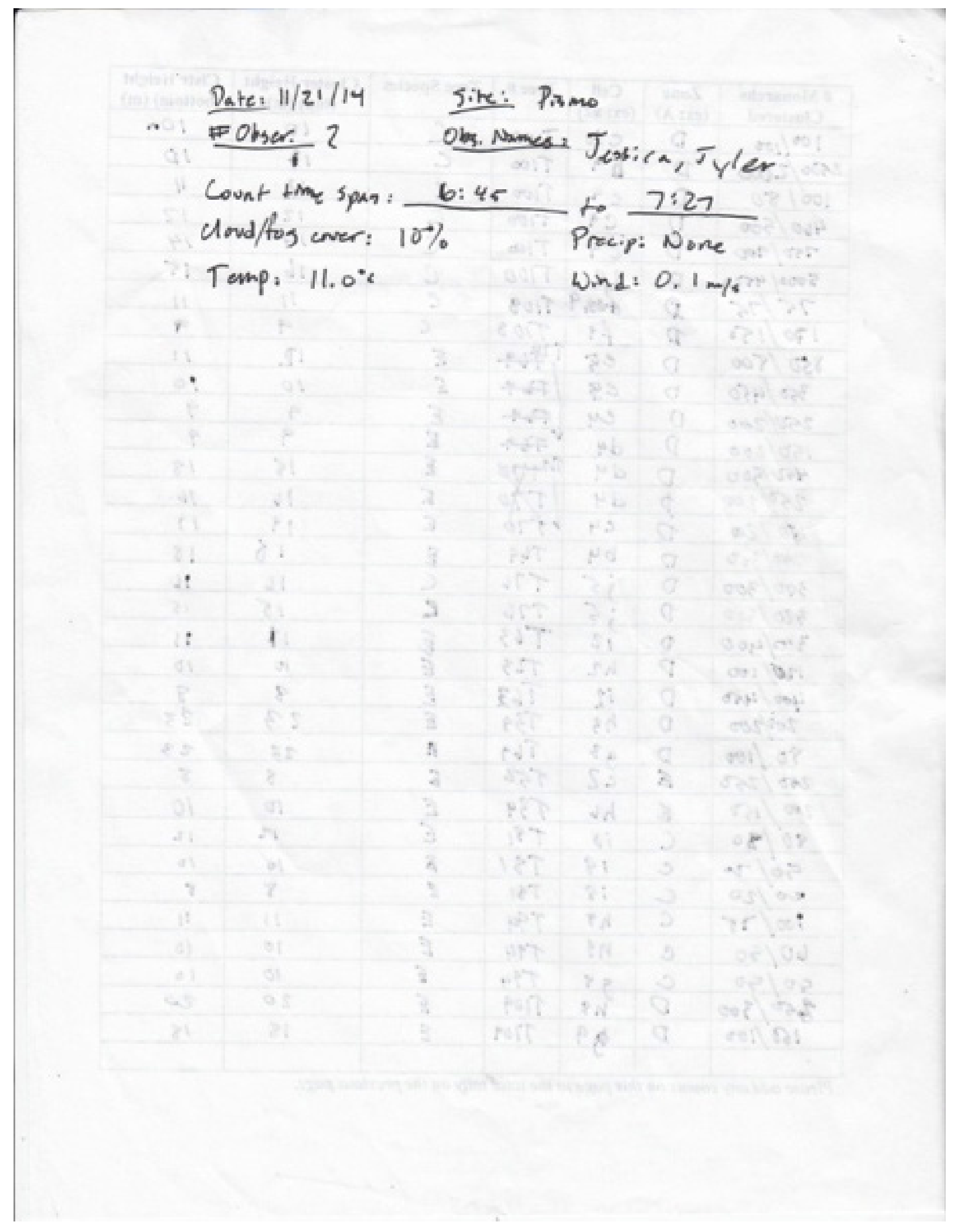




\begin{tabular}{|c|c|c|c|c|c|c|}
\hline $\begin{array}{l}\text { Monarchs } \\
\text { Clustered }\end{array}$ & $\begin{array}{c}\text { Zone } \\
\text { (ex: A) }\end{array}$ & $\begin{array}{c}\text { Cell } \\
(\mathbf{e x}: \mathbf{2} 2)\end{array}$ & Tree 1 & Tree Species & \begin{tabular}{|l} 
Cluster Height \\
(top) (m)
\end{tabular} & $\begin{array}{l}\text { Clstr Height } \\
\text { (bottom) (m) }\end{array}$ \\
\hline $100 / 100$ & D & C 10 & TwoD: & $C$ & 10 & $\because 10 \mathrm{a}$ \\
\hline $2500 / 2000$ & $D$ & $b^{9}$ & $T 100$ & $c$ & 11 & $J D$ \\
\hline $100 / 80$ & D & $c^{9}$ & Troo & $c$ & .12 & 11 \\
\hline $460 / 500$ & $D$ & C 9 & Tion & C & 12 & 12 \\
\hline $750 / 900$ & D & C9 & Tide & C. & 1414 & 14 \\
\hline $5000 / 4000$ & $D$ & $d q$ & $T 100$ & c & 16 & 15 \\
\hline $75 / 75$ & D & $\cos 9$ & T108 & c & 11 & $\mu$ \\
\hline $170 / 150$ & $D$ & $f_{1}$ & Tios & $c$ & 9 & 9 \\
\hline $850 / 800$ & D & es & 1746 & $\varepsilon$ & 12 & 11 \\
\hline $350 / 450$ & D & es & PA & 6 & 10 & 10 \\
\hline $250 / 200$ & $D$ & $\mathrm{cy}$ & R4- & $E$ & 9 & 9 \\
\hline $150 / 200$ & D & $d q$ & 764 & $E$ & 9 & 9 \\
\hline $420 / 500$ & $D$ & $d 4$ & Bh+b & $\bar{\varepsilon}$ & 18 & 18 \\
\hline$2 5 \% \longdiv { 2 0 0 }$ & $D$ & d4 & T/10 & $\varepsilon$ & 16 & 16 \\
\hline $190 / 20$ & 0 & C4 & 770 & $\varepsilon$ & 119 & 19 \\
\hline $200 / 350$ & $D$ & b4 & T6q & $\varepsilon$ & 16 & 18 \\
\hline $300 / 300$ & D & js & 776 & $C$ & 16 & 16 \\
\hline $320 / 300$ & D & $; 5$ & 776 & $c$ & 15 & 15 \\
\hline $300 / 400$ & 0 & 12 & $T 63$ & $E$ & 11 & 11 \\
\hline $120 / 100$ & $D$ & h2 & $\pi / 3$ & $E$ & 10 & 10 \\
\hline $400 / 400$ & $D$ & $i 2$ & 163 & $E$ & 8 & 5 \\
\hline $200 / 200$ & 0 & h3 & 138 & $E$ & 23 & 23 \\
\hline $80 / 100$ & $D$ & $9^{3}$ & T69 & E & 23 & 23 \\
\hline $200 / 250$ & 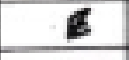 & $c 2$ & 150 & E & 8 & 8 \\
\hline $200 / 150$ & $E$ & he & 134 & $E$ & 10 & 10 \\
\hline $80^{\circ} / 80$ & $C$ & is & $T \nabla_{1}$ & $\epsilon$ & 12 & 12 \\
\hline $50 / 70$ & c & 18 & $T \$ 1$ & $F$ & 10 & 10 \\
\hline $20 / 20$ & c & is & $T \$ 1$ & $\ell$ & 8 & 5 \\
\hline $100 / 7 \%$ & $C$ & hr & $T S 4$ & $E$ & 1 & II \\
\hline $60 / 50$ & c & ns & 194 & $E$ & 10 & 10 \\
\hline $50 / 50$ & c & s5 & $T 94$ & F & 10 & 10 \\
\hline $26^{\circ} / 300$ & $D$ & hs & $\pi / 29$ & $E$ & 20 & 20 \\
\hline $150 / 100$ & D & 99 & Tio9 & $\varepsilon$ & 18 & 15 \\
\hline & & & & & & \\
\hline
\end{tabular}

Please add any counts an this page to the total rally on the previous page. 


\section{Over-wintering Monarch Butterfly Project Monareh Count Data Sheet}

DATE $11 / 22 / 19$ SITE NAME fismio

\# Observers 4 Observer name(s) Handil Eretl, Jeur, baniel

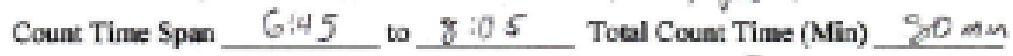

Clood/Fog Cover_O \% Precipituticn (circle one) wone drixhle rain

Temp $\left({ }^{C}\right) \_0.2$ Wind (m/3) 0.2 Wind (Beaufor) __ Wind direction NÉ

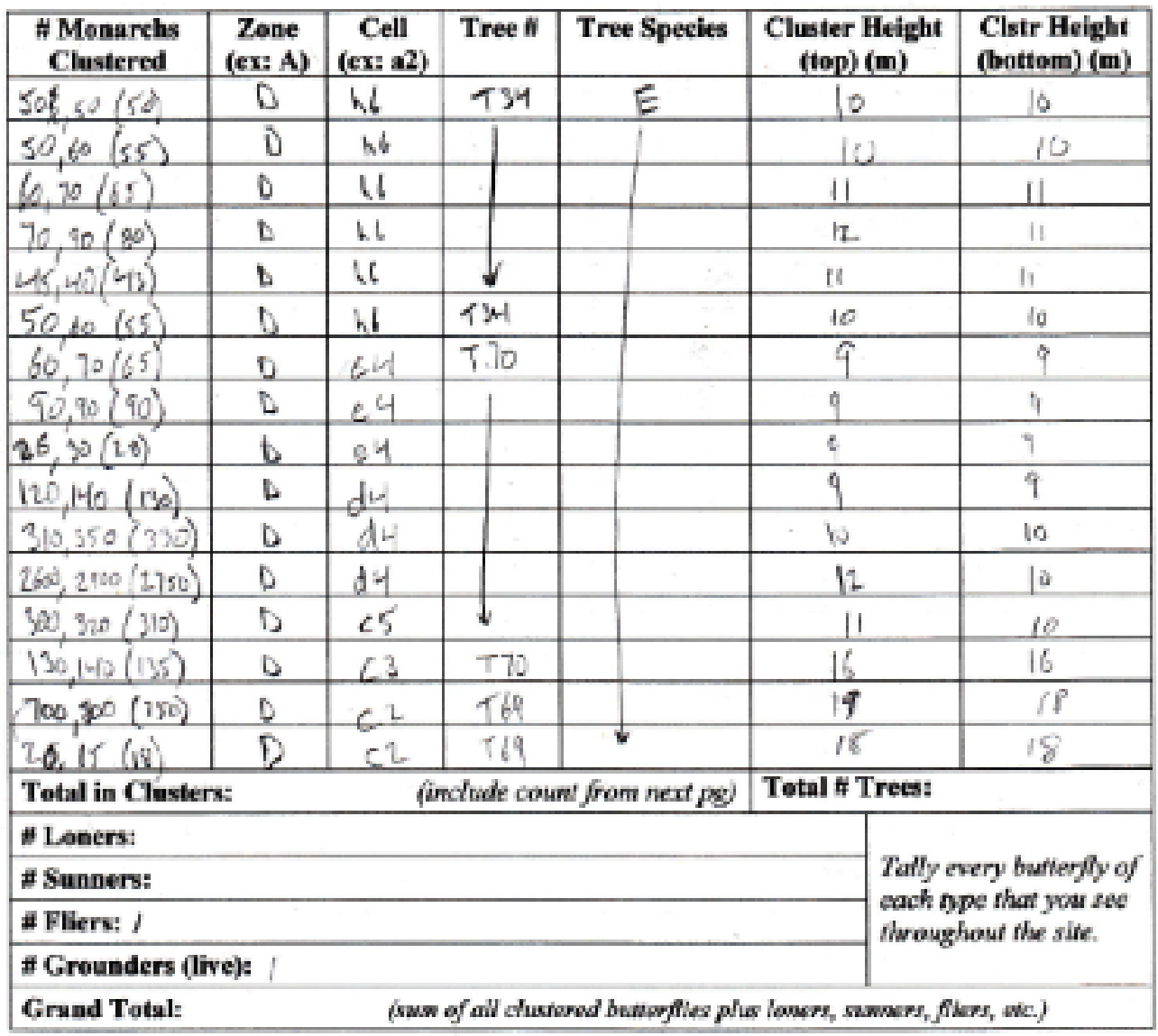

\begin{tabular}{|l|l|}
\hline \# Mating Monarchs: & \\
\hline \# Dead Monarchs: & \\
\hline Other Notes: & \\
\hline
\end{tabular}




\begin{tabular}{|c|c|c|c|c|c|c|}
\hline $\begin{array}{l}\text { Monarehs } \\
\text { Clustered }\end{array}$ & $\begin{array}{l}\text { Zone } \\
\text { (ex: A) }\end{array}$ & $\begin{array}{c}\text { Cell } \\
(\mathbf{e x :}: \mathbf{2})\end{array}$ & Tree II & Tree Species & $\begin{array}{l}\text { Cluster Height } \\
\text { (top) (m) }\end{array}$ & $\begin{array}{l}\text { Clstr Height } \\
\text { (bottom) (m) }\end{array}$ \\
\hline $700,250(2.5)$ & $D$ & $c^{2}$ & $T 69$ & $E$ & 18 & 17 \\
\hline $140,18(185)$ & D & $B 3$ & +69 & & 9 & 9 \\
\hline $700,000(150)^{\prime}$ & 0 & $b_{3}$ & 169 & & 4 & 20 \\
\hline $500,600\left(59^{3}\right)$ & D & $\mathrm{Cl}^{2}$ & $T 69$ & & 21 & 21 \\
\hline $200,30(1000)$ & 0 & i2 & +6 & & 7 & 6 \\
\hline $390,400(395)$ & i) & i2 & T6) & & 9 & $a$ \\
\hline $150,157(50)$ & 0 & $h_{2}$ & 163 & & 8 & 8 \\
\hline $80(0,1+0)$ & $E$ & $C_{2}$ & $T 49$ & & 7 & 7 \\
\hline $120,120(120)$ & D & $h_{2}$ & $T 3_{f}$ & & 25 & 25 \\
\hline & & & & & & \\
\hline & & & & & & \\
\hline & & & & & & \\
\hline & & & & & & \\
\hline & & & & & & \\
\hline & & & & & & \\
\hline & & & & & & \\
\hline & & & & & & \\
\hline & & & & & & \\
\hline & & & & & & \\
\hline & & & & & & \\
\hline & & & & & & \\
\hline & & & & & & \\
\hline & & & & & & \\
\hline & & & & & & \\
\hline & & & & & & \\
\hline & & & & & & \\
\hline & & & & & & \\
\hline & & & & & & \\
\hline & & & & & & \\
\hline & & & & & & \\
\hline & & & & & & \\
\hline
\end{tabular}

Please add any counts on this page to the total tally an the previous page. 


\section{Over-wintering Monareh Butterfly Project Monarch Count Data Sheet}

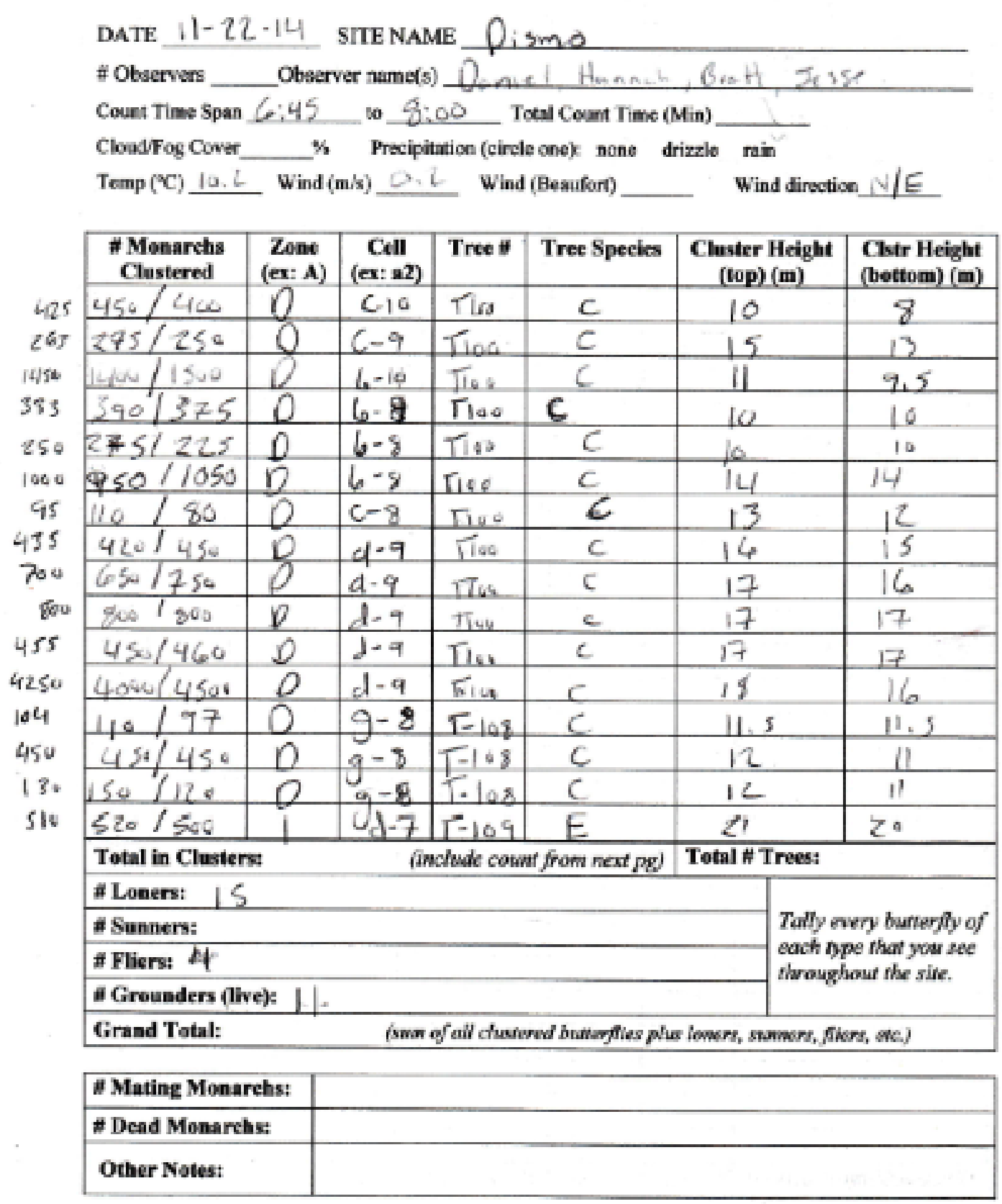




\begin{tabular}{|c|c|c|c|c|c|c|}
\hline $\begin{array}{l}\text { \#onarehs } \\
\text { Clustered }\end{array}$ & $\begin{array}{c}\text { Zone } \\
(e x: A)\end{array}$ & $\begin{array}{c}\text { Cell } \\
(e x: 2)\end{array}$ & Tree W & Tree Species & $\begin{array}{l}\text { Cluster Height } \\
\text { (top) (m) }\end{array}$ & $\begin{array}{l}\text { Clstr Height } \\
\text { (bottem)(m) }\end{array}$ \\
\hline 120,105 & 0 & $d-7$ & $T-109$ & $E$ & 20 & 10 \\
\hline $175 / 15 d$ & C & $i-q$ & $T=81$ & $E$ & 12 & 12 \\
\hline $250 / 300$ & C & $3=3$ & $r-76$ & C & $n+16$ & 16 \\
\hline $70 / 60$ & C & $i-3$ & $T-76$ & c & 16 & 16 \\
\hline $220 / 250$ & c & -3 & $1-76$ & C & 17 & 17 \\
\hline $65 / 75$ & $c$ & -3 & $T=76$ & C & 18 & 18 \\
\hline & - & & & & & \\
\hline & & & & & & \\
\hline & & & & & & \\
\hline & & & & & & \\
\hline & & & & & & \\
\hline & & & & & & \\
\hline & & & & & & \\
\hline & & & & & & \\
\hline & & & & & & \\
\hline & & & & & & \\
\hline & & & & & & \\
\hline & & & & & & \\
\hline & & & & & & \\
\hline 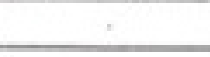 & & & & & & \\
\hline & & & & & & \\
\hline & & & & & & \\
\hline & & & & & & \\
\hline & & & & & & \\
\hline & & & & & & \\
\hline & & & & & & \\
\hline & & & & & & \\
\hline & & & & & & \\
\hline & & & & & & \\
\hline & & & & & & \\
\hline & & & & & & \\
\hline & & & & & & \\
\hline & & & & & & \\
\hline
\end{tabular}




\section{Over-wintering Monarch Butterfyy Project Monarch Count Data Sheet}

DATE $11 / 2>/ 17$ SITE NAME Pismo

a Observers 4 Observer name(s) Enily, Nicele, Doriel, Hannh

Count Time Span 6:45 to 7:35 Total Coent Time (Min) 50

CloudFog Cover_20\% Precipitation (circle one) nong drizzle rain

Temp (C) B.5 Wind (m/s) 2 Wind (Beaufort)__ Wind direction $N$



\begin{tabular}{|l|l|}
\hline \# Mating Monarehs: & \\
\hline inead Monarehs: & \\
\hline Other Notes: & \\
\hline
\end{tabular}




\begin{tabular}{|c|c|c|c|c|c|c|}
\hline $\begin{array}{l}\text { \# Monarchs } \\
\text { Clustered }\end{array}$ & $\begin{array}{c}\text { Zose } \\
(\text { ex: } A)\end{array}$ & $\begin{array}{c}\text { Cell } \\
(\boldsymbol{e x}: \mathbf{2})\end{array}$ & Tree \# & Tree Species & $\begin{array}{l}\text { Cluster Height } \\
\text { (top) (m) }\end{array}$ & $\begin{array}{l}\text { Clstr Height } \\
\text { (bottom) (m) }\end{array}$ \\
\hline $60,90(75)$ & $D$ & M & $T \geqslant 0$ & Ex & 15 & 15 \\
\hline $500,600(550)$ & D & $d y$ & 770 & Euc & 12 & II \\
\hline $450,1500(455)$ & $b$ & $d y$ & 770 & Eve & $n$ & 10 \\
\hline $200,340\left(x_{0}\right)$ & D & $d y$ & 770 & Euc & 9 & 9 \\
\hline $320,350(335)$ & C & 4 & 176 & $C_{4 p}$ & 18 & 17 \\
\hline & & & & & & \\
\hline & & & & & & \\
\hline & & & & & & \\
\hline & & & & & & \\
\hline & & & & & & \\
\hline & & & & & & \\
\hline & & & & & & \\
\hline & & & & & & \\
\hline & & & & & & \\
\hline & & & & & & \\
\hline & & & & & & \\
\hline & & & & & & \\
\hline & & & & & & \\
\hline & & & & & & \\
\hline & & & & & & \\
\hline & & & & & & \\
\hline & & & & & & \\
\hline & & & & & & \\
\hline & & & & & & \\
\hline & & & & & & \\
\hline & & & & & & \\
\hline & & & & & & \\
\hline & & & & & & \\
\hline & & & & & & \\
\hline & & & & & & \\
\hline & & & & & & \\
\hline & & & & & & \\
\hline & & & & & & \\
\hline & & & & & & \\
\hline
\end{tabular}






Please add any counts on this page to the foral tally on the previous page. 
Appendix C

\begin{tabular}{|c|c|c|c|c|c|c|}
\hline$\Delta$ & A & B & C & D & E & $\mathrm{F}$ \\
\hline 1 & Date & MonarchCount & TreeNum & TreeSpecies & Height & ZoneCell \\
\hline 2 & $11 / 1 / 2014$ & 12 & T63 & E & 3 & D-i-1 \\
\hline 3 & $11 / 1 / 2014$ & 45 & T63 & E & 3 & D-i-1 \\
\hline 4 & $11 / 1 / 2014$ & 15 & T63 & E & 3 & D-i-1 \\
\hline 5 & $11 / 1 / 2014$ & 22 & T63 & E & 4 & D-h-1 \\
\hline 6 & $11 / 1 / 2014$ & 45 & T63 & E & 4 & D-h-1 \\
\hline 7 & $11 / 1 / 2014$ & 15 & T63 & $\mathrm{E}$ & 4 & D-h-1 \\
\hline 8 & $11 / 1 / 2014$ & 55 & T63 & E & 4 & D-h-1 \\
\hline 9 & $11 / 1 / 2014$ & 36 & T63 & E & 4 & D-h-1 \\
\hline 10 & $11 / 1 / 2014$ & 28 & T69 & E & 10 & D-e-3 \\
\hline
\end{tabular}

